\title{
DOMESTIC CANONICAL ALGEBRAS AND SIMPLE LIE ALGEBRAS
}

\author{
HIDETO ASASHIBA \\ Dedicated to Professor Claus Michael Ringel on the occasion of his 60th birthday
}

\begin{abstract}
For each simply-laced Dynkin graph $\Delta$ we realize the simple complex Lie algebra of type $\Delta$ as a quotient algebra of the complex degenerate composition Lie algebra $L(A)_{1}^{\mathbb{C}}$ of a domestic canonical algebra $A$ of type $\Delta$ by some ideal $I$ of $L(A)_{1}^{\mathbb{C}}$ that is defined via the Hall algebra of $A$, and give an explicit form of $I$. Moreover, we show that each root space of $L(A)_{1}^{\mathbb{C}} / I$ has a basis given by the coset of an indecomposable $A$-module $M$ with root easily computed by the dimension vector of $M$.
\end{abstract}

\section{Introduction}

Let $A$ be a finite-dimensional algebra over a finite field $k$ with $q$ elements, and consider the free abelian group $\mathcal{H}(A)$ with basis the isoclasses of finite $A$-modules. Then by Ringel [23] $\mathcal{H}(A)$ turns out to be an associative ring with identity, called the integral Hall algebra of $A$, with respect to the multiplication whose structure constants are given by the numbers of filtrations of modules with factors isomorphic to modules that are multiplied (see 2.1). The free abelian subgroup $\bar{L}(A)$ of $\mathcal{H}(A)$ with basis the isoclasses of finite indecomposable $A$-modules becomes a Lie subalgebra modulo $q-1$ whose Lie bracket is given by the commutator of the Hall multiplication. We call this Lie bracket the Hall commutator. It would be interesting to realize all types of simple (complex) Lie algebras using this Hall commutator.

Along this line, Ringel [24] realized the positive part of the simple Lie algebra $\mathfrak{g}(\Delta)$ for each Dynkin type $\Delta$. Further Peng and Xiao [17] realized all types of simple Lie algebras by the so-called root categories of finite-dimensional representation-finite hereditary algebras. But the Lie bracket was not completely given by the Hall commutator, because the root category $\mathcal{R}$ provides only the positive and the negative parts. The Cartan subalgebra $\mathfrak{h}$ was given by a subgroup of the Grothendieck group of $\mathcal{R}$ over the field $\mathbb{Q}$ of rational numbers. The Hall commutator was used to define the Lie bracket only inside $\mathcal{R}$, and when the bracket should not be closed in $\mathcal{R}$, namely when we deal with an indecomposable object $X$ in $\mathcal{R}$ of a root $\alpha$ and an indecomposable object $Y$ in $\mathcal{R}$ of the root $-\alpha$, the definition of the bracket $[X, Y]$ was changed in order to have $[X, Y] \in \mathfrak{h}$. In [1] we succeeded to realize general linear algebras and special linear algebras (see also Iyama [12]) by the Hall commutator defined on cyclic quiver algebras. In this realization also the Cartan subalgebra was naturally provided together with the

2000 Mathematics Subject Classification. Primary 16G20; Secondary 17B20, 17B60.

Key words and phrases. simple Lie algebras, Hall algebras, canonical algebras. 
positive and the negative parts. In [2] we gave a way how to realize all types of simple Lie algebras by the Hall commutator using tame hereditary algebras, in particular we gave an explicit realization of simple Lie algebras of type $D_{n}$.

However this realization needed some rational constants to define a necessary ideal of the Lie algebra. In this paper we give another realization by using domestic canonical algebras. Here we do not use a surjective Lie algebra homomorphism from an affine Lie algebra ([13]) that was an essential tool in [2]. In the realization using a tame hereditary algebra we had to choose some orientation for the quiver of the algebra. But if we use a canonical algebra we are free from choosing an orientation of the quiver of the algebra except for the $A_{n}$ case because the orientation is given from the beginning. For simplicity we deal only with simply-laced cases. Non-simply-laced cases may be treated using the generalized definition of canonical algebras by Ringel [22]. We expect that the same approach works to realize affine Kac-Moody algebras by using tubular canonical algebras instead of domestic ones. (In fact, Zhengxin Chen is carrying out this plan, the primary version [5] contained a similar error as in the first version of this paper.) It should be pointed out that in the realization using canonical algebras the preprojective (resp. preinjective) component contains only basis vectors of the positive (resp. negative) part (see Remarks 4.7 and 8.1), in contrast, in the realization using hereditary (non-canonical) algebras the preprojective component and the preinjective component contain basis elements of both positive and negative parts. Finally we mention that there is a possibility to construct representations of simple Lie algebras by the form of our realization using infinite-dimensional modules as done in [12].

The first version contained a serious error that the constructed Lie algebra may turn out to be zero because the relations required on it was too much. This problem was fixed in the present version.

The paper is organized as follows. After preliminaries in Sect. 1 we collect necessary facts on Lie algebra constructions using Hall algebras, and domestic canonical algebras in sections 2 and 3, respectively. In Sect. 4 we state our main theorem, and Sect. 5 is devoted to preparations of our proof of the main theorem. We give a proof of the main theorem in Sect.6. We next examine root spaces of the Lie algebra constructed here to prove the remaining theorem in Sect. 7. Finally in the last section we exhibit an example of basis vectors of the realization of simple Lie algebra of type $D_{5}$, and an example that shows an error in the first version of the paper.

\section{Preliminaries}

1.a. Notation. Throughout this paper $k$ is a finite field of cardinality $q \geq 3$. When we deal with domestic canonical algebras of type $E_{8}$ (see Sect. 3.a for definition) we assume that char $k \neq 2$. For a (finite-dimensional) $k$-algebra $A$, we denote by $\bmod A$ the category of finite-dimensional (left) $A$-modules, and by ind $A$ the full subcategory of $\bmod A$ consisting of indecomposable modules. For an $A$-module $M, \operatorname{top} M:=M / \operatorname{rad} M$, soc $M, l(M)$ and $[M]$ denote the top, the socle, the composition length and the isoclass of $M$, respectively. For $\mathcal{C}=\bmod A$, ind $A$ we denote by $[\mathcal{C}]$ the set of isoclasses of objects in $\mathcal{C}$. For a field extension $K$ of $k$, we set $V^{K}:=V \otimes_{k} K$ for all $k$-vector spaces $V$. For a set $E,|E|$ denotes the cardinality of $E$. The set of positive integers and the 
set of non-negative integers are denoted by $\mathbb{N}$ and by $\mathbb{N}_{0}$, respectively. For a ring $R$, $R^{\times}$denotes the set of invertible elements of $R$. By $\delta_{i j}$ we denote the Kronecker symbol, i.e., $\delta_{i j}=1$ if $i=j$, and $\delta_{i j}=0$ if $i \neq j$. For an abelian group $L$, we set $L^{\mathbb{C}}:=L \otimes_{\mathbb{Z}} \mathbb{C}$ and $L^{\mathbb{Q}}:=L \otimes_{\mathbb{Z}} \mathbb{Q}$. For elements $x_{1}, \ldots, x_{n}$ of a Lie algebra, we set

$$
\left[x_{1}, \ldots, x_{n}\right]:=\left[\left[\cdots\left[\left[x_{1}, x_{2}\right], x_{3}\right], \cdots\right], x_{n}\right] .
$$

For Auslander-Reiten theory we refer to [3, 7, 21] and for tilting theory to [10, 21, 9]. We set $\Gamma_{A}$ to be the Auslander-Reiten quiver of $A$. $D=\operatorname{Hom}_{k}(-, k)$ and $\tau=\tau_{A}$ denote the usual $k$-duality $\bmod A \rightarrow \bmod A^{\text {op }}$ and the Auslander-Reiten translation of $A$, respectively.

1.b. Hall numbers. For $X, Y, Z \in \bmod A$, we set

$$
\begin{aligned}
& \mathcal{F}_{X, *}^{Z}=\{M \mid M \text { is a submodule of } Z \text { with } Z / M \cong X\}, \\
& \mathcal{F}_{*, Y}^{Z}=\{M \mid M \text { is a submodule of } Z \text { with } M \cong Y\}, \\
& \mathcal{F}_{X Y}^{Z}=\mathcal{F}_{X, *}^{Z} \cap \mathcal{F}_{*, Y}^{Z}
\end{aligned}
$$

and the cardinalities of these are denoted by $F_{X, *}^{Z}, F_{*, Y}^{Z}$, and $F_{X Y}^{Z}$, respectively. $F_{X Y}^{Z}$ is called a Hall number. If $X \cong X^{\prime}, Y \cong Y^{\prime}$ and $Z \cong Z^{\prime}$ in $\bmod A$, then we clearly have $F_{X Y}^{Z}=F_{X^{\prime} Y^{\prime}}^{Z^{\prime}}$. Therefore we may set $F_{[X][Y]}^{[Z]}:=F_{X Y}^{Z}$. Recall the following well-known formula (the Riedtmann formula) for $A$-modules $X, Y$ and $Z$ (see [20, 4.1, 4.3], [16, Lemma 3.1]):

$$
F_{X Y}^{Z}=\frac{\left|\operatorname{Ext}_{A}^{1}(X, Y)_{Z}\right| \cdot\left|\operatorname{Aut}_{A} Z\right|}{\left|\operatorname{Hom}_{A}(X, Y)\right| \cdot\left|\operatorname{Aut}_{A} X\right| \cdot\left|\operatorname{Aut}_{A} Y\right|} \text { in } \mathbb{Z}
$$

where $\operatorname{Ext}_{A}^{1}(X, Y)_{Z}$ is the set of equivalence classes in $\operatorname{Ext}_{A}^{1}(X, Y)$ of extensions with the middle term $Z$. To compute the number $F_{X Y}^{Z}$ we will use the number

$$
W_{X Y}^{Z}:=\mid\left\{(f, g) \in \operatorname{Hom}_{A}(Y, Z) \times \operatorname{Hom}_{A}(Z, X) \mid 0 \rightarrow Y \stackrel{f}{\longrightarrow} Z \stackrel{g}{\longrightarrow} X \rightarrow 0 \text { is exact }\right\} \mid \text {. }
$$

As easily seen we have the following relationship between $F_{X Y}^{Z}$ and $W_{X Y}^{Z}$ :

$$
F_{X Y}^{Z}=\frac{W_{X Y}^{Z}}{\left|\operatorname{Aut}_{A} X\right| \cdot\left|\operatorname{Aut}_{A} Y\right|} .
$$

\section{1.c. Representations of quivers.}

Definition 1.1. (1) Recall that a quiver is a quadruple $Q=\left(Q_{0}, Q_{1}, t_{Q}, h_{Q}\right)$, where $Q_{0}, Q_{1}$ are sets (or classes) and $t_{Q}, h_{Q}$ are maps from $Q_{1}$ to $Q_{0}$. Elements of $Q_{0}, Q_{1}$ are called vertices and arrows of $Q$, respectively, and for each $\alpha \in Q_{1}$ the vertices $t_{Q}(\alpha), h_{Q}(\alpha)$ are called the tail and the head of $\alpha$, respectively. By drawing an arrow $t_{Q}(\alpha) \stackrel{\alpha}{\longrightarrow} h_{Q}(\alpha)$ for each $\alpha \in Q_{1}$ we can express $Q$ as an oriented graph. We can regard categories as quivers by forgetting compositions.

(2) A morphism from a quiver $Q$ to a quiver $Q^{\prime}$ is a pair $f=\left(f_{0}, f_{1}\right)$ of maps $f_{i}: Q_{i} \rightarrow Q_{i}^{\prime}$ for $i=0$ and 1 such that $f_{0} t_{Q}=t_{Q^{\prime}} f_{1}$ and $f_{0} h_{Q}=h_{Q^{\prime}} f_{1}$. This is also written as $f=(f(x), f(\alpha))_{x \in Q_{0}, \alpha \in Q_{1}}$, where we put $f(x):=f_{0}(x), f(\alpha):=f_{1}(\alpha)$ for each $x \in Q_{0}$ and $\alpha \in Q_{1}$. 
(3) A $k$-representation of a quiver $Q$ is just a morphism $V=(V(x), V(\alpha))_{x, \alpha}$ from $Q$ to the category $\bmod k$ of finite-dimensional $k$-vector spaces regarded as a quiver. The definition of morphisms between representations of $Q$ is similar to that of natural transformations between functors.

Remark 1.2. Unless otherwise stated we only deal with finite quivers, i.e. quivers with only finitely many vertices and arrows.

The vector space $k Q$ with basis the set of all paths in $Q$ turns out to be a $k$ algebra with identity via the multiplication given by concatenation of paths. We refer to [7] for details. When an algebra $A$ is defined by a quiver $Q$ with relations $\rho_{1}, \ldots, \rho_{t}$, say $A=k Q / I$, where $I$ is the admissible ideal of $k Q$ generated by $\rho_{1}, \ldots, \rho_{t}$, we identify $\bmod A$ with the category of representations of $Q$ satisfying the relations $\rho_{1}, \ldots, \rho_{t}$ as in [7]. Thus for an $A$-module $M$, regarded as a $k$-representation, we write $M=(M(x), M(\alpha))_{x \in Q_{0}, \alpha \in Q_{1}}$. In fact, $M(x)=\mathbf{e}_{x} M$ and $M(\alpha)$ is given by the left multiplication by $\alpha+I \in A$.

Remark 1.3. For simplicity we assume throughout the rest of this paper that $A$ is a finite-dimensional $k$-algebra defined by a quiver $Q=\left(Q_{0}, Q_{1}, t_{Q}, h_{Q}\right)$ with relations.

Definition 1.4. For each vertex $x$ of $Q$, we denote by $\mathbf{e}_{x}$ the idempotent of $A$ corresponding to $x$. The support algebra of an $A$-module $M$, denoted by $\operatorname{supp} M$, is defined by

$$
\operatorname{supp} M:=A / A \mathbf{e}_{M} A,
$$

where $\mathbf{e}_{M}:=\sum_{M(x)=0} \mathbf{e}_{x}$. Note that $\bmod \operatorname{supp} M$ forms a full subcategory of $\bmod A$ closed under extensions.

\section{1.d. Grothendieck group.}

Definition 1.5. (1) The image of an $M \in \bmod A$ in the Grothendieck group $K_{0}(A)$ of $A$ is denoted by $\underline{\operatorname{dim}} M$ and is called the dimension vector of $M$.

(2) For each vertex $x \in Q_{0}$ we set $S_{x}:=A \mathbf{e}_{x} / \operatorname{rad} A \mathbf{e}_{x}$ to be the simple $A$-module corresponding to $x$, and $e_{x}:=\underline{\operatorname{dim}} S_{x}$.

(3) For each $v, w \in K_{0}(A)$ we write $v \leq w$ if $v_{x} \leq w_{x}$ for all $x \in Q_{0}$. This defines a partial order on $K_{0}(A)$. Further we write $v<w$ if $v \leq w$ but $v \neq w$.

Remark 1.6. (1) The set $\left\{e_{x} \mid x \in Q_{0}\right\}$ forms a basis of $K_{0}(A)$, by which we regard each element $v=\sum_{x \in Q_{0}} v_{x} e_{x}$ in $K_{0}(A)$ as a row vector $\left(v_{x}\right)_{x \in Q_{0}} \in \mathbb{Z}^{Q_{0}}$, and identify $K_{0}(A)$ with $\mathbb{Z}^{Q_{0}}$. Under this identification we have $\underline{\operatorname{dim}} M=\left(\operatorname{dim}_{k} M(x)\right)_{x \in Q_{0}}$. Note that since we deal with row vectors, each $\mathbb{Z}$-endomorphism $f$ of $K_{0}(A)$ is expressed by the right multiplication by the corresponding matrix $F$ as $f(v)=v F$ for all $v \in K_{0}(A)$.

(2) Let $K$ be an arbitrary field extension of $k$ and consider the $K$-algebra $A^{K}:=$ $A \otimes_{k} K$. By identifying $K_{0}\left(A^{K}\right)$ with $\mathbb{Z}^{Q_{0}}$ by the same way as above we also have $\underline{\operatorname{dim}} M^{K}=\left(\operatorname{dim}_{K} M^{K}(x)\right)_{x \in Q_{0}}$. Then since $\operatorname{dim}_{k} M(x)=\operatorname{dim}_{K} M^{K}(x)$ for all $x \in Q_{0}$, we have

$$
\underline{\operatorname{dim}} M^{K}=\underline{\operatorname{dim}} M .
$$


Definition 1.7. Since the derived category $\mathcal{D}^{\mathrm{b}}(\bmod A)$ of bounded complexes in $\bmod A$ is a triangulated category, the Grothendieck group $K_{0}\left(\mathcal{D}^{\mathrm{b}}(\bmod A)\right)$ is defined by using triangles $([8],[9,1.1])$. For each $X \in \mathcal{D}^{\mathrm{b}}(\bmod A)$ we denote by $\underline{\operatorname{dim}}^{D} X$ the image of $X$ in $K_{0}\left(\mathcal{D}^{\mathrm{b}}(\bmod A)\right)$.

Remark 1.8. We regard $\bmod A$ as a subcategory of $\mathcal{D}^{\mathrm{b}}(\bmod A)$ by the canonical embed$\operatorname{ding} \bmod A \rightarrow \mathcal{D}^{\mathrm{b}}(\bmod A)$ sending modules to complexes concentrated in degree zero, which induces an isomorphism $K_{0}(A) \rightarrow K_{0}\left(\mathcal{D}^{\mathrm{b}}(\bmod A)\right), \underline{\operatorname{dim}} X \mapsto \underline{\operatorname{dim}}^{D} X$ with the inverse $\underline{\operatorname{dim}}^{D} X \mapsto \sum_{i=1}^{\infty}(-1)^{i} \underline{\operatorname{dim}} X^{i}([8])$. By this isomorphism we identify $K_{0}(A)$ with $K_{0}\left(\mathcal{D}^{\mathrm{b}}(\overline{\bmod } A)\right)$. Therefore for all $X \in \mathcal{D}^{\mathrm{b}}(\bmod A)$ we may write $\underline{\operatorname{dim}} X=\underline{\operatorname{dim}}^{D} X$, and we have

$$
\underline{\operatorname{dim}} X=\sum_{i=1}^{\infty}(-1)^{i} \underline{\operatorname{dim}} X^{i} .
$$

In particular, for each $X \in \mathcal{D}^{\mathrm{b}}(\bmod A)$ and $i \in \mathbb{Z}$ we have

$$
\underline{\operatorname{dim}} X[i]=(-1)^{i} \underline{\operatorname{dim}} X .
$$

1.e. Bilinear form and quadratic form. Let $C$ be the Cartan matrix of $A$, namely the matrix whose $(i, j)$-entry is given by $\operatorname{dim} \mathbf{e}_{i} A \mathbf{e}_{j}$ for all $i, j \in Q_{0}$ (Definition 1.4).

Definition 1.9. If the global dimension of $A$ is finite, say at most $d \in \mathbb{N}$, then $C$ is invertible and we can define a bilinear form $B_{A}$ by

$$
B_{A}(v, w)=v C^{-T} w^{T}
$$

for all $v, w \in K_{0}(A) \cong \mathbb{Z}^{Q_{0}}\left(C^{-T}\right.$ denotes the inverse matrix of the transposed matrix $C^{T}$ of $\left.C\right)$.

Remark 1.10. In the setting above the following is well-known:

$$
B_{A}(\underline{\operatorname{dim}} X, \underline{\operatorname{dim}} Y)=\sum_{i=0}^{d}(-1)^{i} \operatorname{dim} \operatorname{Ext}_{A}^{i}(X, Y)
$$

for all $A$-modules $X, Y([21$, Lemma 2.4]).

Definition 1.11. (1) We denote by $\chi_{A}$ the corresponding quadratic form, namely

$$
\chi_{A}(v):=B_{A}(v, v)
$$

for all $v \in K_{0}(A)$.

(2) An element $v \in K_{0}(A)$ is called a root (resp. a radical) of $\chi_{A}$ if $\chi_{A}(v)=1$ (resp. $\left.\chi_{A}(v)=0\right)$.

(3) We set $\operatorname{rad} \chi_{A}:=\left\{v \in K_{0}(A) \mid \chi_{A}(v)=0\right\}$ and call it the radical of $\chi_{A}$. 
1.f. Exceptional modules. Recall that an $A$-module $X$ is called exceptional if $X$ is indecomposable and $\operatorname{Ext}_{A}^{1}(X, X)=0$. We take an algebraic closure $\bar{k}$ of $k$, and set $\Omega=\Omega_{A}$ to be the set of all finite field extensions $K$ of $k$ contained in $\bar{k}$ such that $\left(\operatorname{End}_{A} X\right)^{K}$ is a field for all exceptional $A$-modules $X$. We set $\mathcal{E}_{\text {ex }}(A):=\left\{\operatorname{End}_{A}(X) \mid\right.$ $X$ is exceptional $\} / \cong$ and $\mathcal{E}(A):=\left\{\operatorname{End}_{A}(X) \mid X\right.$ is simple $\} / \cong$. (In the simply-laced cases our domestic canonical algebras $A$ defined in the next section are defined by quivers with relations and we always have $\mathcal{E}(A)=\{k\}$. Therefore in our case we can omit this notation, but we keep it here because it is needed in the non-simply-laced cases and it tells us how to generalize our argument.)

Lemma 1.12. If $A$ is an algebra derived equivalent to a hereditary algebra $H$, then $\mathcal{E}(A) \subseteq \mathcal{E}_{e x}(A) \subseteq \mathcal{E}_{e x}(H)=\mathcal{E}(H)$. Therefore in particular, $\Omega_{A}$ is an infinite set.

Proof. Since simple modules are exceptional, both $\mathcal{E}(A) \subseteq \mathcal{E}_{\text {ex }}(A)$ and $\mathcal{E}(H) \subseteq \mathcal{E}_{\text {ex }}(H)$ are trivial. In the hereditary case it is known that the converse inclusion is also true ([27]), thus we have $\mathcal{E}(H)=\mathcal{E}_{\text {ex }}(H)$. We only have to show that $\mathcal{E}_{\text {ex }}(A) \subseteq \mathcal{E}_{\text {ex }}(H)$. Let $F: \mathcal{D}^{\mathrm{b}}(\bmod A) \rightarrow \mathcal{D}^{\mathrm{b}}(\bmod H)$ be a triangle-equivalence, and $X$ an exceptional $A$-module. Then $F X[i] \in \bmod H$ for some $i \in \mathbb{Z}$ because $F X$ is an indecomposable complex and $H$ is hereditary. It is obvious from the construction that $F X[i]$ is an exceptional $H$-module. The algebra isomorphisms $\operatorname{End}_{A}(X) \cong \mathcal{D}^{\mathrm{b}}(\bmod A)(X, X) \cong$ $\mathcal{D}^{\mathrm{b}}(\bmod H)(F X, F X) \cong \operatorname{End}_{H}(F X[i])$ show that $\mathcal{E}_{e x}(A) \subseteq \mathcal{E}_{e x}(H)$.

\section{Lie algebras defined by the Hall multiplication}

2.a. Hall algebras. Since $A$ is a finite-dimensional $k$-algebra with $k$ a finite field, $A$ is a finitary ring as shown in Ringel [23], i.e., $\operatorname{Ext}_{A}^{1}(X, Y)$ is a finite group for all $X, Y \in \bmod A$.

Definition 2.1. The free abelian group $\mathcal{H}(A)$ with basis $\left\{u_{[X]}\right\}_{[X] \in[\bmod A]}$ together with the multiplication defined by

$$
u_{[X]} u_{[Y]}:=\sum_{[Z] \in[\bmod A]} F_{[X][Y]}^{[Z]} u_{[Z]}
$$

is called the integral Hall algebra of $A$.

Ringel [23] proved the following.

Lemma 2.2. $\mathcal{H}(A)$ is an associative ring with the identity $1=u_{[0]}$.

\section{2.b. Lie algebras.}

Definition 2.3. Let $\bar{L}(A)$ be the free abelian subgroup of $\mathcal{H}(A)$ with basis $\left\{u_{\alpha}\right\}_{\alpha \in[\text { ind } A]}$. We set $L /(a):=L / a L$ for all $\mathbb{Z}$-modules $L$ and $a \in \mathbb{Z}$, and denote elements $x+a \bar{L}(A)$ of $\bar{L}(A) /(a)(x \in \bar{L}(A))$ simply by $x$.

We have the following by Ringel [26, Proposition 3] (see also Ringel [25, Proposition $1])$. 
Lemma 2.4. The free $\mathbb{Z} /(q-1) \mathbb{Z}$-module $\bar{L}(A) /(q-1)$ is a Lie subalgebra of $\mathcal{H}(A) /(q-$ 1) with the Lie bracket

$$
\left[u_{[X]}, u_{[Y]}\right]=\sum_{[Z] \in[\operatorname{ind} A]}\left(F_{[X][Y]}^{[Z]}-F_{[Y][X]}^{[Z]}\right) u_{[Z]}
$$

for each $[X],[Y] \in[$ ind $A]$.

Note that in the right hand side of the formula above the sum may be taken only over $[Z] \in[$ ind $A]$ such that $\underline{\operatorname{dim}} Z=\underline{\operatorname{dim}} X+\underline{\operatorname{dim}} Y$. Hence if we put $(\bar{L}(A) /(q-1))_{d}$ to be the free $\mathbb{Z} /(q-1) \mathbb{Z}$-submodule with the basis $\left\{u_{[X]} \mid[X] \in[\operatorname{ind} A], \underline{\operatorname{dim}} X=d\right\}$ for all $d \in K_{0}(A)$, we have $\left[(\bar{L}(A) /(q-1))_{d},(\bar{L}(A) /(q-1))_{e}\right] \subseteq(\bar{L}(A) /(q-1))_{d+e}$ for all $d, e \in K_{0}(A)$. Thus we have the following.

Proposition 2.5. $\bar{L}(A) /(q-1)=\bigoplus_{d \in K_{0}(A)}(\bar{L}(A) /(q-1))_{d}$ is a $K_{0}(A)$-graded Lie algebra.

\section{2.c. Composition Lie algebras.}

Definition 2.6. For $A$-modules $X, Y$ and $Z$ a polynomial $\varphi_{Z X}^{Y}(T) \in \mathbb{Z}[T]$ in an indeterminate $T$ with integral coefficients is called a Hall polynomial for the triple $(X, Y, Z)$ if $F_{Z^{K} X^{K}}^{Y^{X}}=\varphi_{Z X}^{Y}(|K|)$ for all $K \in \Omega_{A}$.

Note that when the set $\Omega_{A}$ is infinite, a Hall polynomial for a triple is uniquely determined if it exists.

Definition 2.7. Assume that $\Omega_{A}$ is an infinite set. By Lemma 2.b, $\bar{L}\left(A^{K}\right) /(|K|-1)$ is a Lie subalgebra of $\mathcal{H}\left(A^{K}\right) /(|K|-1)$ over $\mathbb{Z} /(|K|-1) \mathbb{Z}$ for each $K \in \Omega$. Consider the Lie algebra over $\mathbb{Z}$ given by the direct product of Lie algebras:

$$
\Pi=\Pi_{A}:=\prod_{K \in \Omega} \bar{L}\left(A^{K}\right) /(|K|-1) .
$$

(1) An $A$-module $X$ is called absolutely indecomposable (with respect to $\Omega$ ) if

$$
X^{K} \text { is indecomposable for all } K \in \Omega \text {. }
$$

We write $\mathbf{u}_{[X]}:=\left(u_{\left[X^{K}\right]}\right)_{K \in \Omega} \in \Pi$ if $X$ is absolutely indecomposable. Note that all simple modules are absolutely indecomposable.

(2) The Lie subalgebra of $\Pi$ generated by $\left\{\mathbf{u}_{[S]} \mid S\right.$ is simple $\}$ is denoted by $L(A)_{1}$ and is called the degenerate composition Lie algebra of $A$. The Lie algebra $L(A)_{1}$ is not a torsion $\mathbb{Z}$-module because $\Omega$ is an infinite set.

Lemma 2.8. Let $X, Y, Z$ be absolutely indecomposable A-modules with $\underline{\operatorname{dim}} X+$ $\underline{\operatorname{dim}} Z=\underline{\operatorname{dim}} Y$ such that $Y$ is the unique indecomposable A-module with dimension vector $\underline{\operatorname{dim} X}+\underline{\operatorname{dim}} Z$ up to isomorphisms. If there exist Hall polynomials $\varphi_{Z X}^{Y}$ and $\varphi_{X Z}^{Y}$, then

in $\Pi$.

$$
\left[\mathbf{u}_{Z}, \mathbf{u}_{X}\right]=\left(\varphi_{Z X}^{Y}(1)-\varphi_{X Z}^{Y}(1)\right) \mathbf{u}_{Y}
$$

Proof. This follows from $F_{Z^{K} X^{K}}^{Y^{K}}=\varphi_{Z X}^{Y}(1)$ in $\mathbb{Z} /(|K|-1) \mathbb{Z}$ for all $K \in \Omega_{A}$. 


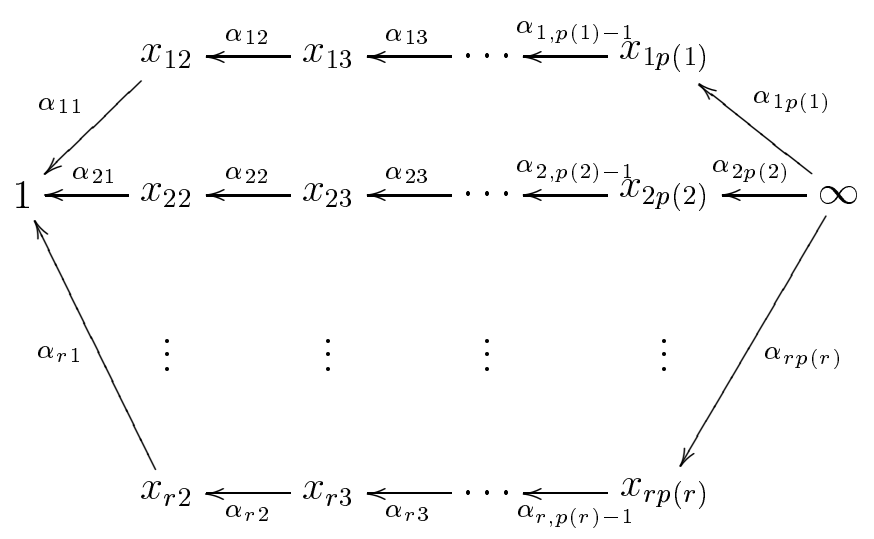

FiguRE 3.1. Quiver of a canonical algebra

The following seems to be well-known.

Proposition 2.9. Let $\Delta$ be a simply-laced Dynkin graph. If $A$ is a connected representationfinite hereditary algebra of type $\Delta$, and $M$ an indecomposable A-module. Then $\mathbf{u}_{M} \in$ $L(A)_{1}$.

Proof. Since in this case $\Omega_{A}$ is an infinite set, $L(A)_{1}$ is defined. We prove the assertion by induction on $\operatorname{dim} M$. If $\operatorname{dim} M=1$, then $M$ is simple, and $\mathbf{u}_{M} \in L(A)_{1}$. Assume that $\operatorname{dim} M>1$. Then as easily seen there exists a simple $A$-module $S$ and an indecomposable $A$-module $N$ such that $\underline{\operatorname{dim}} M=\underline{\operatorname{dim}} S+\underline{\operatorname{dim}} N$. By putting $a:=\varphi_{S N}^{M}(1)-\varphi_{N S}^{M}(1)$ we have $\left[\mathbf{u}_{S}, \mathbf{u}_{N}\right]=a \mathbf{u}_{M}$ in $\Pi$ by Lemma 2.8. Here by [24] precisely one of the two values $\varphi_{S N}^{M}(1)$ and $\varphi_{N S}^{M}(1)$ is nonzero, and the nonzero value is in $\{ \pm 1, \pm 2, \pm 3\}$. Therefore $a \in\{ \pm 1, \pm 2, \pm 3\}$. Since $\Delta$ is simply-laced, we have $a= \pm 1$. Hence $\mathbf{u}_{M}=\frac{1}{a}\left[\mathbf{u}_{S}, \mathbf{u}_{N}\right] \in L(A)_{1}$ because by induction hypothesis $\mathbf{u}_{N} \in L(A)_{1}$.

\section{Canonical algebras}

3.a. Canonical algebras. Among canonical algebras we consider, in this paper, only domestic canonical algebras given by quivers with relations. Namely, a domestic canonical algebra $A$ is given by the quiver $Q$ in Figure [3.1, where $r \in\{2,3\}$, $p(i) \geq p(i+1) \geq 1$ for all $1 \leq i \leq r-1$, with no relation when $r=2$; and with the relation $\sum_{i=1}^{r} \alpha_{i 1} \cdots \alpha_{i p(i)}=0$ when $r=3$. Further when $r=3$ it is assumed that

$$
(p(1), p(2), p(3)) \in\{(d, 2,2),(3,3,2),(4,3,2),(5,3,2)\}
$$

for some $d \geq 2$. For convenience we set $x_{11}=x_{21}=\cdots=x_{r 1}=1, x_{1, p(1)+1}=$ $x_{2, p(2)+1}=\cdots=x_{r, p(r)+1}=\infty$, and give a partial order on $Q_{0}$ by setting $x_{i j}<x_{i, j+1}$ for all $1 \leq i \leq r$ and $1 \leq j \leq p(i)$. Denote by $Q^{l}$ the quiver obtained from $Q$ by deleting the vertex $\infty$. Note that the underlying graph $\Delta$ of $Q^{l}$ is a (simply-laced) Dynkin graph, which is called the type of $A$. Conversely every simply-laced Dynkin graph $\Gamma$ is obtained in this way, and the canonical algebra of the type $\Gamma$ is uniquely determined if $\Gamma$ is not of type $A_{n}$. We set $\Delta_{0}:=Q_{0}^{l}=Q_{0} \backslash\{\infty\}$ to be the set of vertices 
of $\Delta, n:=\left|\Delta_{0}\right|$, and denote by $\left(a_{x y}\right)_{x, y \in \Delta_{0}}$ the Cartan matrix expressed by the graph $\Delta$, namely

$$
a_{x y}= \begin{cases}2 & \text { if } x=y ; \\ -1 & \text { if } x \neq y, \text { and } x, y \text { are neighbors in } \Delta ; \text { and } \\ 0 & \text { if } x \neq y, \text { and } x, y \text { are not neighbors in } \Delta,\end{cases}
$$

where vertices $x, y \in \Delta_{0}$ are said to be neighbors in $\Delta$ if they are connected by an edge in $\Delta$.

Throughout the rest of this paper we assume that $A$ is a domestic canonical algebra.

3.b. Domestic canonical algebras as tame concealed algebras. Note that $\Gamma_{A}$ has a preprojective component $([21$, p.80]), which contains a unique complete slice $\mathcal{S}$ ([10, 7.1], cf. [21, p.180]) with $P_{\infty}:=A \mathbf{e}_{\infty}$ the unique source. Let $T$ be the corresponding slice module ([21, p.183]), which is a tilting module for $A$. Then $H:=\operatorname{End}_{A}(T)^{\text {op }}$ is a tame hereditary algebra, whose quiver is obtained by giving an orientation to the affine graph $\Delta^{(1)}$ corresponding to the type $\Delta$ of $A$. Thus $A$ is a tilted algebra ([10], [21, 4.2]) or more precisely a tame concealed algebra ([21, 4.3]), and hence the global dimension of $A$ is at most 2 , and it is derived equivalent to the hereditary algebra $H$ by [9, Theorem 2.10] or [19, Theorem 6.4]. Denote by $\mathcal{F}$ and $\mathcal{T}$ (resp. $\mathcal{Y}$ and $\mathcal{X}$ ) the torsion-free class and the torsion class in $\bmod A(\operatorname{resp} . \bmod H)$, respectively, defined by the tilting module $T$. Note that the torsion pair $(\mathcal{T}, \mathcal{F})$ splits, i.e., we have a disjoint union ind $A=($ ind $A \cap \mathcal{T}) \sqcup($ ind $A \cap \mathcal{F})$, whereas in general the torsion pair $(\mathcal{X}, \mathcal{Y})$ does not split, thus ind $H \supsetneq$ (ind $H \cap \mathcal{X}) \sqcup$ (ind $H \cap \mathcal{Y}$ ). Set

$$
\begin{gathered}
F:=\operatorname{Hom}_{A}(T,-), F^{\prime}:=\operatorname{Ext}_{A}^{1}(T,-), \hat{F}:=\mathbf{R} \operatorname{Hom}_{A}^{*}(T,-), \\
G:=T \otimes_{H}-, G^{\prime}:=\operatorname{Tor}_{1}^{H}(T,-), \hat{G}:=T \stackrel{\mathbf{L}}{\otimes_{H}-}
\end{gathered}
$$

Then as well-known we have quasi-inverse pairs of equivalences and triangle-equivalences

$$
\mathcal{T} \underset{G}{\stackrel{F}{\rightleftarrows}} \mathcal{Y}, \mathcal{F} \underset{G^{\prime}}{\stackrel{F^{\prime}}{\rightleftarrows}} \mathcal{X} \text {, and } \mathcal{D}^{\mathrm{b}}(\bmod A) \underset{\hat{G}}{\stackrel{\hat{F}}{\rightleftarrows}} \mathcal{D}^{\mathrm{b}}(\bmod H) .
$$

Since $H$ is given by a quiver, we have $\mathcal{E}(H)=\{k\}$. Then by Lemma 1.12 we have the following.

Lemma 3.1. $\mathcal{E}_{\text {ex }}(A)=\mathcal{E}(A)=\{k\}$, and $\Omega_{A}$ is an infinite set.

3.c. Bilinear form, quadratic form and rank. Since the global dimension of $A$ is finite (Sect. 3.b) , the bilinear form $B:=B_{A}$ is defined (Definition 1.9). Denote by $r_{T}: K_{0}(A) \rightarrow K_{0}(H)$ the isomorphism defined by $r_{T}(\underline{\operatorname{dim}} X)=\underline{\operatorname{dim}} \hat{F} X$ for all $X \in$ $\mathcal{D}^{\mathrm{b}}(\bmod A)$. Then as well-known $B_{A}(x, y)=B_{H}\left(r_{T}(x), r_{T}(y)\right)$ for all $x, y \in K_{0}(A)$, in particular, we have $\chi_{A}(x)=\chi_{H}\left(r_{T}(x)\right)$ for all $x \in K_{0}(A)$ ([9, Proposition III.1.5]). Thus $\operatorname{rad} \chi_{A}$ is isomorphic to $\operatorname{rad} \chi_{H}$, which is well-known to be a free abelian group of rank 1 . Then since $\delta:=(1,1, \ldots, 1) \in \operatorname{rad} \chi_{A}$, we have $\operatorname{rad} \chi_{A}=\mathbb{Z} \delta$. Thus $\delta$ is the minimal positive radical vector of $\chi_{A}$. 
We set $\rho:=(1,0, \ldots, 0,-1) \in \mathbb{Z}^{n+1}$. For an element $v \in K_{0}(A) \cong \mathbb{Z}^{n+1}$ we set

$$
\operatorname{rank} v:=v_{1}-v_{\infty}=v \rho^{T}
$$

and call it the rank of $v$, and for an $A$-module $M$ we set

$$
\operatorname{rank} M:=\operatorname{rank}(\underline{\operatorname{dim}} M)=\operatorname{dim} M(1)-\operatorname{dim} M(\infty)=(\underline{\operatorname{dim}} M) \rho^{T},
$$

which is called the rank of $M$.

A direct calculation shows that

$$
B(v, w)= \begin{cases}\sum_{x \in Q_{0}} v_{x} w_{x}-\sum_{x \rightarrow y} v_{x} w_{y} & \text { if } \Delta \in\left\{A_{n} \mid n \in \mathbb{N}\right\} \\ \sum_{x \in Q_{0}} v_{x} w_{x}-\sum_{x \rightarrow y} v_{x} w_{y}+v_{\infty} w_{1} & \text { if } \Delta \notin\left\{A_{n} \mid n \in \mathbb{N}\right\}\end{cases}
$$

for all $v, w \in K_{0}(A)$, where the sum $\sum_{x \rightarrow y}$ is taken over all pairs $(x, y) \in Q_{0} \times Q_{0}$ such that there exists an arrow from $x$ to $y$ in $Q$. This immediately yields

$$
\begin{aligned}
& B(\delta, v)=-\operatorname{rank} v \\
& B(v, \delta)=\operatorname{rank} v
\end{aligned}
$$

for all $v \in K_{0}(A)$.

3.d. Lost indecomposable modules. Since $H$ is hereditary, each indecomposable complex in $\mathcal{D}^{\mathrm{b}}(\bmod H)$ is isomorphic to a complex concentrated in one degree. In other words each indecomposable complex in $\mathcal{D}^{\mathrm{b}}(\bmod H)$ is regarded as an $H$-module up to shifts. But the corresponding statement does not hold for $A$ in general. An indecomposable $H$-module $X$ is sent by $\hat{G}$ to a complex of $A$-modules that cannot be isomorphic to an $A$-module up to shifts if and only if $X \notin \mathcal{X} \cup \mathcal{Y}$. Thus, when we pass from $\bar{L}(H)_{(q-1)}$ to $\bar{L}(A)_{(q-1)}$ we lose the basis $u_{[X]}$ for such an $X$. Therefore $L(A)_{1}^{\mathbb{C}}$ would not realize the positive part of the affine Kac-Moody algebra of type $\Delta^{(1)}$, which was realized as $L(H)_{1}^{\mathbb{C}}$ by a part of [18, Theorem 4.7] (see also [25, Theorems 2 and 3]). In this connection it would be interesting to know which indecomposable complex of $A$-modules can be an $A$-module up to shifts. This is the case if and only if positive and negative entries are not mixed in its dimension vector. Namely, we have the following.

Lemma 3.2. Let $X \in \mathcal{D}^{b}(\bmod A)$ be indecomposable. Then $X[i] \in$ ind $A$ for some $i \in \mathbb{Z}$ if and only if $\underline{\operatorname{dim}} X>0$ or $\underline{\operatorname{dim}} X<0$.

Proof. $(\Rightarrow)$. If $X[i] \in \operatorname{ind} A$ for some $i \in \mathbb{Z}$, then $0<\underline{\operatorname{dim}} X[i]=(-1)^{i} \underline{\operatorname{dim}} X$ by $\underline{1 . d}$, Hence $\underline{\operatorname{dim}} X>0$ or $\underline{\operatorname{dim}} X<0$.

$(\Leftarrow)$. Since the torsion pair $(\mathcal{F}, \mathcal{T})$ in 3 .b splits, $X[i] \in$ ind $A$ if and only if $X[i] \in \mathcal{F}$ or $X[i] \in \mathcal{T}$ for all indecomposable complexes $X \in \mathcal{D}^{\mathrm{b}}(\bmod A)$ and $i \in \mathbb{Z}$.

Now assume that $X[i] \notin$ ind $A$ for all $i \in \mathbb{Z}$. It is enough to show that $\underline{\operatorname{dim}} X \ngtr 0$ and $\underline{\operatorname{dim}} X \nless 0$. Since $H$ is hereditary, there exists some $i \in \mathbb{Z}$ such that $Y:=\hat{F} X[i] \in$ $\bmod H$. It follows from the assumption that $X[i] \notin \mathcal{F}$ and $X[i] \notin \mathcal{T}$. Therefore $Y \notin \mathcal{Y}$ and $Y \notin \mathcal{X}$. Let $\mathcal{P}_{H}, \mathcal{R}_{H}$, and $\mathcal{I}_{H}$ be the preprojective component, the tubular family, 
and the preinjective component of the Auslander-Reiten quiver $\Gamma_{H}$ of $H$, respectively. Since $\mathcal{P}_{H}, \mathcal{R}_{H} \subseteq \mathcal{Y}$, we have $Y \in \mathcal{I}_{H}$. Consider the canonical exact sequence

$$
0 \rightarrow Y^{\prime} \stackrel{\mu}{\longrightarrow} Y \stackrel{\varepsilon}{\longrightarrow} Y^{\prime \prime} \rightarrow 0
$$

with $Y^{\prime} \in \mathcal{X}$ and $Y^{\prime \prime} \in \mathcal{Y}$. Then

$$
\begin{aligned}
\underline{\operatorname{dim}} X[i] & =\underline{\operatorname{dim}} \hat{G} Y \\
& =\underline{\operatorname{dim}} \hat{G} Y^{\prime}+\underline{\operatorname{dim}} \hat{G} Y^{\prime \prime} \\
& =\underline{\operatorname{dim}} G Y^{\prime}-\underline{\operatorname{dim}} G^{\prime} Y^{\prime}+\underline{\operatorname{dim}} G Y^{\prime \prime}-\underline{\operatorname{dim}} G^{\prime} Y^{\prime \prime} \\
& =\underline{\operatorname{dim}} G Y^{\prime \prime}-\underline{\operatorname{dim}} G^{\prime} Y^{\prime} .
\end{aligned}
$$

Hence

$$
\underline{\operatorname{dim}} X[i]=\underline{\operatorname{dim}} G Y^{\prime \prime}-\underline{\operatorname{dim}} G^{\prime} Y^{\prime} .
$$

We first show that $(\underline{\operatorname{dim}} X[i])_{1}<0$. Let $I_{1}$ be the injective hull of $S_{1}$. Suppose that $\operatorname{Hom}_{H}\left(Y^{\prime \prime}, F I_{1}\right) \neq 0$. Then since $\operatorname{Hom}_{H}\left(\varepsilon, F I_{1}\right): \operatorname{Hom}_{H}\left(Y, F I_{1}\right) \rightarrow \operatorname{Hom}_{H}\left(Y^{\prime \prime}, F I_{1}\right)$ is an epimorphism, we have $\operatorname{Hom}_{H}\left(Y, F I_{1}\right) \neq 0$. This shows that $Y$ is a predecessor of $F I_{1}$ in $\mathcal{I}_{H}$. Since $F I_{1} \in \mathcal{Y}$ and $\mathcal{Y} \cap \mathcal{I}_{H}$ is closed under predecessors in $\mathcal{I}_{H}$, we have $Y \in \mathcal{Y}$, a contradiction. Therefore we must have $\operatorname{Hom}_{H}\left(Y^{\prime \prime}, F I_{1}\right)=0$. Then since $Y^{\prime \prime}, F I_{1} \in \mathcal{Y}$, we have $\operatorname{Hom}_{H}\left(G Y^{\prime \prime}, I_{1}\right)=0$, which shows that $\left(\underline{\operatorname{dim}} G Y^{\prime \prime}\right)_{1}=0$. Whereas since $G^{\prime} Y^{\prime} \in \mathcal{F} \subseteq \mathcal{P}$, we have $\left(\underline{\operatorname{dim}} G^{\prime} Y^{\prime}\right)_{1}>\left(\underline{\operatorname{dim}} G^{\prime} Y^{\prime}\right)_{\infty} \geq 0$. Hence by (3-3) we have $(\underline{\operatorname{dim}} X[i])_{1}<0$. We next show that $(\underline{\operatorname{dim}} X[i])_{\infty}>0$. Since $Y^{\prime \prime} \in \mathcal{Y} \cap \mathcal{I}_{H}$, we have $G Y^{\prime \prime} \in \mathcal{I}$, and hence $\left(\underline{\operatorname{dim}} G Y^{\prime \prime}\right)_{\infty}>\left(\underline{\operatorname{dim}} G Y^{\prime \prime}\right)_{1} \geq 0$. Further since $G^{\prime} Y^{\prime}$ is not a successor of $P_{\infty}$, we have $\left(\underline{\operatorname{dim}} G^{\prime} Y^{\prime}\right)_{\infty}=0$. Hence by $(\underline{3-3})$ we have $(\underline{\operatorname{dim}} X[i])_{\infty}>0$. As a consequence we have $\underline{\operatorname{dim}} X[i] \ngtr 0$ and $\underline{\operatorname{dim}} X[i] \nless 0$, which implies that $\underline{\operatorname{dim}} X \ngtr 0$ and $\underline{\operatorname{dim}} X \nless 0$ by Remark 1.8 .

3.e. Indecomposable modules of dimension vector $\boldsymbol{\delta}$. Let $K \in \Omega$. We list indecomposable $A^{K}$-modules with dimension vector $\delta$ for later use.

(1) For each $c \in K$ we define a $A^{K}$-module $W_{c}(K)$ as follows. Let $W_{c}(K)(x)=K$ for all $x \in Q_{0}$; and

$$
W_{c}(K)\left(\alpha_{i j}\right)=\left\{\begin{aligned}
c \mathbb{1} & \text { if }(i, j)=(2,1) ; \\
-(1+c) \mathbb{1} & \text { if }(i, j)=(3,1) ; \text { and } \\
\mathbb{1} & \text { otherwise. }
\end{aligned}\right.
$$

(2) For each arrow $\alpha=\alpha_{i j} \in Q_{1}$ we define a $A^{K}$-module $X_{\alpha}(K)=X_{i j}(K)$ as follows. Let $X_{\alpha}(K)(x)=X_{i j}(K)(x)=k$ for all $x \in Q_{0}$; and

$$
X_{i j}(K)\left(\alpha_{s t}\right)=\left\{\begin{aligned}
0 & \text { if }(s, t)=(i, j) ; \\
-\mathbb{1} & \text { if }(i, s, t) \in\{(1,3,1),(2,3,1),(3,2,1)\} ; \text { and } \\
\mathbb{1} & \text { otherwise. }
\end{aligned}\right.
$$

Note that $W_{0}(K)=X_{21}(K)$ and that when $A$ is not of type $A_{n}$, we have $W_{-1}(K)=$ $X_{31}(K)$. For $K=k$ we simply write $W_{c}=W_{c}(k)$ and $X_{i j}=X_{i j}(k)$ for all $c \in k$ and $\alpha_{i j} \in Q_{1}$. Then clearly we have $X_{i j}(K) \cong X_{i j}^{K}$ for all $\alpha_{i j} \in Q_{1}$ and $K \in \Omega$. The following is well-known ([21]). 
Proposition 3.3. The set $\left\{W_{c}(K), X_{i j}^{K} \mid c \in K \backslash E_{\Delta}, \alpha_{i j} \in Q_{1}\right\}$ forms a complete set of representatives of isoclasses of indecomposable $A^{K}$-modules with dimension vector $\delta$, where

$$
E_{\Delta}:= \begin{cases}\{0\} & \text { if } A \text { is of type } A_{n} ; \text { and } \\ \{0,-1\} & \text { otherwise. }\end{cases}
$$

3.f. The Auslander-Reiten quiver. Recall that the set of isoclasses of simple regular representations of the Kronecker algebra $\left(\begin{array}{cc}k & 0 \\ k^{2} & k\end{array}\right)$ over $k$ is identified with the projective line $\mathbb{P}^{1}(k)=\operatorname{Proj} k\left[x_{0}, x_{1}\right]$ of the ring $k$, which is needed to apply general results in [22]. We obtain the following by [21, Theorem 4.3], [22, Theorem 1], and [21, Theorem 3.7] (see [21] for definitions of orbit quivers, tubular families and so on):

Theorem 3.4. Let $A$ be a domestic canonical algebra. Then

(1) $\Gamma_{A}$ consists of a unique preprojective component $\mathcal{P}$ with orbit quiver of type $\Delta^{(1)}$ containing all projective indecomposables, a unique preinjective component $\mathcal{I}$ with orbit quiver of type $\Delta^{(1)}$ containing all injective indecomposables and a stable separating tubular $\mathbb{P}^{1}(k)$-family $\mathcal{R}=\left(\mathcal{T}_{c}\right)_{c \in \mathbb{P}^{1}(k)}$ of type $(p(1), \ldots, p(r))$ separating $\mathcal{P}$ from $\mathcal{I}$ (see Definition 3.5 for definition);

(2) An indecomposable $A$-module $M$ is preprojective, i.e., $M \in \mathcal{P}$ (resp. preinjective, i.e., $M \in \mathcal{I})$ if and only if $\operatorname{rank} M>0$ (resp. $<0)$, if and only if all maps $M(\alpha)$, $\alpha \in Q_{1}$ are monomorphisms (resp. epimorphisms) and there is some non-isomorphism among them; and $M$ is regular, i.e., $M \in \mathcal{R}$ if and only if rank $M=0$, if and only if either all maps $M(\alpha), \alpha \in Q_{1}$ are isomorphisms, or there is some non-monomorphism and some non-epimorphism among them; and

(3) $\bmod A$ is controlled by $\chi_{A}$. Namely,

(a) $\left\{\chi_{A}(\underline{\operatorname{dim}} X) \mid X \in \operatorname{ind} A\right\}=\{0,1\}$;

(b) for any positive root $v$ of $\chi_{A}|\{[X] \in[\operatorname{ind} A] \mid v=\underline{\operatorname{dim}} X\}|=1$; and

(c) for any positive radical vector $v$ of $\chi_{A}|\{[X] \in[\operatorname{ind} A] \mid v=\underline{\operatorname{dim}} X\}| \geq|k|+1$.

More detailed account on the tubular $\mathbb{P}^{1}(k)$-family $\mathcal{R}$ will be given in Sect. 3.g below.

Definition 3.5. (1) For each positive root $v$ of $\chi_{A}$ we denote by $m(v)$ the unique element of $\{[X] \in$ [ind $A] \mid v=\underline{\operatorname{dim}} X\}$ and choose an indecomposable $A$-module $M(v) \in m(v)$. For each $K \in \Omega$ we set $m(v)^{K}:=\left[M(v)^{K}\right]$.

(2) We here recall the definition for $\mathcal{R}$ to be separating $\mathcal{P}$ from $\mathcal{I}$. First for a translation subquiver $\mathcal{T}$ of $\Gamma_{A}$ we denote by $\langle\mathcal{T}\rangle$ the full subcategory of $\bmod A$ consisting of the modules in $\mathcal{T}$ (sometimes we simply write $\mathcal{T}$ for $\langle\mathcal{T}\rangle$ if there seems to be no confusion). Then $\langle\mathcal{T}\rangle$ is said to be standard if it is isomorphic to the mesh category $k(\mathcal{T})$ of $\mathcal{T}([21$, p. 51]). Now $\mathcal{R}$ is said to be separating $\mathcal{P}$ from $\mathcal{I}$ if

(a) $\langle\mathcal{R}\rangle$ is standard (thus there are no nonzero morphisms between distinct tubes, and $\left\langle\mathcal{T}_{c}\right\rangle \cong k\left(\mathcal{T}_{c}\right)$ for all $\left.c \in \mathbb{P}^{1}(k)\right)$;

(b) $\operatorname{Hom}_{A}(\mathcal{I}, \mathcal{P})=\operatorname{Hom}_{A}(\mathcal{I}, \mathcal{T})=\operatorname{Hom}_{A}(\mathcal{T}, \mathcal{P})=0$; and

(c) For each $f \in \operatorname{Hom}_{A}(\mathcal{P}, \mathcal{I})$ and each $c \in \mathbb{P}^{1}(k), f$ can be factored through $\mathcal{T}_{c}$.

Corollary 3.6. Let $M$ be an indecomposable A-module. Then

$$
\min _{x \in Q_{0}} \operatorname{dim} M(x)= \begin{cases}\operatorname{dim} M(\infty) & \text { if } M \text { is preprojective } \\ \operatorname{dim} M(1) & \text { if } M \text { is preinjective }\end{cases}
$$


Proof. This is immediate from Theorem $3.4(2)$.

Corollary 3.7. Let $v$ be a root of $\chi_{A}$ and $t \in \mathbb{Z}$. Then $v+t \delta$ is a root of $\chi_{A}$. In particular, $|\operatorname{rank} X| \leq 6$ for all $X \in$ ind $A$.

Proof. By the formula (3-2) we have $\chi_{A}(v+t \delta)=\chi_{A}(v)+t B(v, \delta)+t B(\delta, v)+t^{2} \chi_{A}(\delta)=$ $1+t \operatorname{rank} v-t \operatorname{rank} v+0=1$. Thus $v+t \delta$ is a root of $\chi_{A}$. Now let $X \in \operatorname{ind} A$. If $X$ is regular, then the assertion is trivial because $\operatorname{rank} X=0$. If $X$ is preprojective, then $\operatorname{rank} X>0$ and $\underline{\operatorname{dim}} X$ is a positive root, and hence $w:=\underline{\operatorname{dim}} X-\operatorname{dim} X(\infty) \delta$ is a positive root by Corollary 3.6. Thus there exists some $Y \in$ ind $A$ such that $\underline{\operatorname{dim}} Y=w$. Then $\operatorname{rank} X=\operatorname{rank} Y=\operatorname{dim} Y(1)$ because $\operatorname{dim} Y(\infty)=0$ by construction. Here $Y$ is regarded as an indecomposable module over $\operatorname{supp} Y$ that is a representation-finite hereditary algebra defined by a quiver. Hence $\operatorname{dim} Y(1) \leq 6$ by Gabriel's Theorem [6] on the classification of representation-finite quivers (or Ovsienko's Theorem [15] explained in [21, 1.0 Theorem 1]). If $X$ is preinjective, then the similar argument works to have $-6 \leq \operatorname{rank} X<0$.

3.g. Tubular family. We describe the tubular $\mathbb{P}^{1}(k)$-family $\mathcal{R}=\left(\mathcal{T}_{c}\right)_{c \in \mathbb{P}^{1}(k)}$ in Theorem 3.4 in more detail following [21, 22]. Recall first that as a set of points, $\mathbb{P}^{1}(k)$ decomposes into a disjoint union $\mathbb{P}^{1}(k)=\bigsqcup_{d \in \mathbb{N}} \mathbb{P}^{1}(k)_{d}$ of the subsets

$$
\mathbb{P}^{1}(k)_{d}:=\left\{\langle p\rangle \in \mathbb{P}^{1}(k) \mid p \in k\left[x_{0}, x_{1}\right] \text { is homogeneous, irreducible, and } \operatorname{deg} p=d\right\},
$$

where $d \in \mathbb{N}$. In [2], to parameterize indecomposable modules with dimension vector the minimal positive imaginary root of a simple Lie algebra considered there, we used the set $\mathbb{P}_{k}^{1}:=(k \times k \backslash\{(0,0)\}) / \sim$, where for each $(a, b),\left(a^{\prime}, b^{\prime}\right) \in k \times k \backslash\{(0,0)\}$ we define $(a, b) \sim\left(a^{\prime}, b^{\prime}\right)$ if and only if $(a, b)=t\left(a^{\prime}, b^{\prime}\right)$ for some $t \in k^{\times}$, which is an equivalence relation on $k \times k \backslash\{(0,0)\}$. We here identify $\mathbb{P}_{k}^{1}$ with the subset $\mathbb{P}^{1}(k)_{1}$ of $\mathbb{P}^{1}(k)$ by the bijection $(a: b) \mapsto\left(a x_{0}+b x_{1}\right)$, where $(a: b)$ denotes the equivalence class in $\mathbb{P}_{k}^{1}$ containing $(a, b) \in k \times k \backslash\{(0,0)\}$. We also identify $\mathbb{P}_{k}^{1}$ with the set $k \cup\{\infty\}$ by the bijection $(a: 1) \mapsto a$ for $a \in k$ and $(1: 0) \mapsto \infty$. For each $c \in \mathbb{P}^{1}(k), \mathcal{T}_{c}$ has the following shape: If $c \notin E_{\Delta} \cup\{\infty\}$ (see (3-4)), then $\mathcal{T}_{c}$ is a homogeneous tube, i.e., is isomorphic to the translation quiver $\mathbb{Z} A_{\infty} /\langle\tau\rangle$ (see [21, Chap. 3], it is denoted by $\mathbb{Z} A_{\infty} / 1$ there). The module $W_{c}$ defined in Sect.3.e is the unique module on the mouth of $\mathcal{T}_{c}([21,3.1])$. Each module in $\mathcal{T}_{c}$ is uniquely determined by $W_{c}$ and by its quasilength (= the number of modules in the shortest path from the mouth to it) $m$, and thus we denote it by $W_{c}[m]$. (Since $W_{c}$ is of quasi-length 1 , we can write $W_{c}=W_{c}[1]$.) The set of modules in $\mathcal{T}_{c}$ is equal to $\left\{W_{c}[m] \mid m \in \mathbb{N}\right\}$. Then we have

$$
\underline{\operatorname{dim}} W_{c}[m]=m d \delta \text {, }
$$

if $c \in \mathbb{P}^{1}(k)_{d}$ with $d \in \mathbb{N}$. Next assume that $c \in E_{\Delta} \cup\{\infty\}$, which depends on the value of $r \in\{2,3\}$ in Figure 3.1. Set $c(1)=\infty, c(2)=0$ (and $c(3):=-1$ when $r=3)$. Then for $i=1, \ldots, r, \mathcal{T}_{c(i)}$ is a stable tube of rank $p(i)$, namely, it is isomorphic to the translation quiver $\mathbb{Z} A_{\infty} /\left\langle\tau^{p(i)}\right\rangle\left(=\mathbb{Z} A_{\infty} / p(i)\right.$ in [21, Chap. 3]). The simple modules $S_{x_{i 2}}, \ldots, S_{x_{i p(i)}}$, and the module $W_{c(i)}^{\prime}$ are the modules on the mouth of $\mathcal{T}_{c(i)}$, where $W_{c(i)}^{\prime}:=M\left(\delta-\sum_{j=2}^{p(i)} e_{x_{i j}}\right.$ ) (see Definition 3.5 for the notation), which is possible because a direct calculation shows that $\delta-\sum_{j=2}^{p(i)} e_{x_{i j}}$ is a 
positive root of $\chi_{A}$. Each module in $\mathcal{T}_{c(i)}$ is uniquely determined by its quasi-length $m$ and the starting point $W \in\left\{S_{x_{i 2}}, \ldots, S_{x_{i p(i)}}, W_{c(i)}^{\prime}\right\}$ of the shortest path from the mouth to it. Therefore we denote it by $W[\mathrm{~m}]$. The set of modules in $\mathcal{T}_{c(i)}$ is equal to $\left\{W[m] \mid W \in\left\{S_{x_{i 2}}, \ldots, S_{x_{i p(i)}}, W_{c(i)}^{\prime}\right\}, m \in \mathbb{N}\right\}$. The modules $X_{i j}, j \in\{1, \ldots, p(i)\}$ defined in Sect. 3.e are in $\mathcal{T}_{c(i)}$ and of quasi-length $p(i)$, thus $X_{i j}=W[p(i)]$ for some $W \in\left\{S_{x_{i 2}}, \ldots, S_{x_{i p(i)}}, W_{c(i)}^{\prime}\right\}$. By the additivity of dimension vectors on exact sequences, we easily see that

$$
\underline{\operatorname{dim}} W[d p(i)]=d \delta
$$

for all $d \in \mathbb{N}$, and $\underline{\operatorname{dim}} W[m] \in \mathbb{Z} \delta$ if and only if $m \in \mathbb{Z} p(i)$. In particular, we have $\left\{c \in \mathbb{P}^{1}(k) \mid \mathcal{T}_{c}\right.$ contains a module of dimension vector $\left.\delta\right\}=\mathbb{P}^{1}(k)_{1}=\mathbb{P}_{k}^{1}$. We call $\mathcal{T}_{c(i)}$, $i \in\{1, \ldots, r\}$ non-homogeneous tubes.

3.h. $\tau$-orbits in the preprojective component. Set $\Phi:=-C^{-T} C$ to be the $C o x$ eter matrix of $A$. For later use we give an explicit form of $\Phi^{-1}$ when $A$ is not of type $A_{n}$ :

$$
\Phi^{-1}=\left(\begin{array}{c|cccc|cccc|c|c}
0 & 0 & -1 & \cdots & -1 & 0 & -1 & \cdots & -1 & 0 & -1 \\
\hline 0 & 0 & 1 & 0 & \cdots & 0 & 0 & \cdots & 0 & 0 & 0 \\
\vdots & \vdots & \ddots & \ddots & \ddots & \vdots & \vdots & \ddots & \vdots & \vdots & \vdots \\
0 & 0 & \cdots & 0 & 1 & 0 & 0 & \cdots & 0 & 0 & 0 \\
1 & 0 & \cdots & 0 & 0 & 1 & 1 & \cdots & 1 & 1 & 1 \\
\hline 0 & 0 & \cdots & 0 & 0 & 0 & 1 & 0 & \cdots & 0 & 0 \\
\vdots & \vdots & \ddots & \vdots & \vdots & \vdots & \ddots & \ddots & \ddots & \vdots & \vdots \\
0 & 0 & \cdots & 0 & 0 & 0 & \cdots & 0 & 1 & 0 & 0 \\
1 & 1 & \cdots & 1 & 1 & 0 & \cdots & 0 & 0 & 1 & 1 \\
\hline 1 & 1 & \cdots & 1 & 1 & 1 & \cdots & 1 & 1 & 0 & 1 \\
\hline-2 & -1 & \cdots & -1 & -1 & -1 & \cdots & -1 & -1 & -1 & -1
\end{array}\right),
$$

where the rows and columns are ordered by the sequence $\left(1, x_{12}, \ldots, x_{1 p(1)}, x_{22}, \ldots\right.$, $\left.x_{2 p(2)}, x_{32}, \infty\right)$, and in the first row the two zeros between entries with value -1 correspond to $x_{22}$ and $x_{32}$, whereas in the first column the 1 between zeros corresponds to $x_{1 p(1)}$. In many cases $\Phi^{-1}$ can be seen as a "shadow" of $\tau^{-1}$ in $K_{0}(A)$ as the following statement shows (see [21, $2.4\left(4^{*}\right)$ ] for the proof).

Lemma 3.8. Let $M$ be an A-module. If injdim $M \leq 1$ and $\operatorname{Hom}_{A}\left(D\left(A_{A}\right), M\right)=0$, then

$$
\underline{\operatorname{dim}} \tau^{-1} M=(\underline{\operatorname{dim}} M) \Phi^{-1} .
$$

In order to check that the injective dimension is at most 1 we cite the following lemma from [21].

Lemma 3.9. Let $M$ be an $A$-module. Then injdim $M \leq 1$ if and only if $\operatorname{Hom}_{A}\left(\tau^{-1} M, A\right)=$ 0 . In particular injdim $M \leq 1$ holds if $M$ is an indecomposable module such that $\tau^{-1} M$ is not a predecessor of any projective indecomposable A-module. 
Note that if $M$ is an indecomposable module that is a successor of a complete slice of the preprojective component, then $\tau^{-1} M$ cannot be a predecessor of any projective indecomposable $A$-module, and hence injdim $M \leq 1$.

Direct calculation shows the following.

Lemma 3.10. $\delta \Phi^{-1}=\delta$ and $\Phi^{-1} \rho^{T}=\rho^{T}$.

On the set of dimension vectors of indecomposable preprojective $A$-modules there are two natural partitions: the $\tau^{-1}$-orbit decomposition and the coset decomposition modulo $\delta$. The following gives a relationship between them, which was obtained in answering a question by A. Hubery.

Proposition 3.11. If $M$ is an indecomposable preprojective $A$-module such that $\tau^{-1} M$ is not a predecessor of any projectives in $\Gamma_{A}$, then there exist $t, m \in \mathbb{N}$ such that

$$
\underline{\operatorname{dim}} \tau^{-t} M=\underline{\operatorname{dim}} M+m \delta .
$$

Proof. Let $\mathcal{P}$ be the set of vertices of the preprojective component of $\Gamma_{A}$. For each $r \in\{1,2, \ldots, 6\}$ set

$$
\begin{aligned}
\mathcal{P}_{r} & :=\left\{X \in \mathcal{P} \mid \tau^{-1} X \text { is not a predecessor of any projectives, rank } X=r\right\} \\
\underline{\operatorname{dim}} \mathcal{P}_{r} & :=\left\{\underline{\operatorname{dim}} X \mid X \in \mathcal{P}_{r}\right\} .
\end{aligned}
$$

Define an equivalence relation $\sim$ on $\underline{\operatorname{dim}} \mathcal{P}_{r}$ by $v \sim w$ if and only if $v-w \in \mathbb{Z} \delta$ for all $v, w \in \underline{\operatorname{dim}} \mathcal{P}_{r}$. Since $\underline{\operatorname{dim}} X-\operatorname{dim} X(\infty) \delta$ is a root of $\chi_{A}$ for each $X \in \mathcal{P}_{r}$, there exists

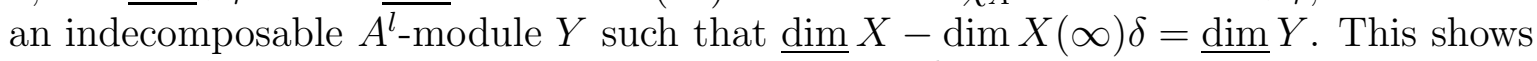
that the quotient set $\left(\underline{\operatorname{dim}} \mathcal{P}_{r}\right) / \sim$ is finite because $A^{l}$ is representation-finite. We show that $\Phi^{-1}$ acts on the finite set $\left(\underline{\operatorname{dim}} \mathcal{P}_{r}\right) / \sim$. Let $X \in \mathcal{P}_{r}$. Then clearly $\tau^{-2} X$ is not a predecessor of any projectives, either, and $\operatorname{rank} \tau^{-1} X=(\underline{\operatorname{dim}} X) \Phi^{-1} \rho^{T}=\operatorname{rank} X=r$ by Lemmas 3.8 and 3.10, Hence $\tau^{-1}$ induces an injective map $\mathcal{P}_{r} \rightarrow \mathcal{P}_{r}$. Thus by Lemma 3.8 the right multiplication by $\Phi^{-1}$ induces an injective map $\underline{\operatorname{dim}} \mathcal{P}_{r} \rightarrow \underline{\operatorname{dim}} \mathcal{P}_{r}$, and by Lemma $\left[3.10\right.$ it also induces an injective map $\left(\underline{\operatorname{dim}} \mathcal{P}_{r}\right) / \sim \rightarrow\left(\underline{\operatorname{dim}} \mathcal{P}_{r}\right) / \sim$, which is a bijection because the set $\left(\underline{\operatorname{dim}} \mathcal{P}_{r}\right) / \sim$ is finite. Now let $M$ be as in the assertion and put $r:=\operatorname{rank} M$. Then $\underline{\operatorname{dim}} M \in \underline{\operatorname{dim}} \mathcal{P}_{r}$ and $(\underline{\operatorname{dim}} M) \Phi^{-t} \sim \underline{\operatorname{dim}} M$ for some $t \in \mathbb{N}$, which means that $\underline{\operatorname{dim}} \tau^{-t} M=\underline{\operatorname{dim}} M+m \delta$ for some $m \in \mathbb{Z}$. If $m=0$, then $\underline{\operatorname{dim}} \tau^{-t} M=\underline{\operatorname{dim}} M$, and we have $\tau^{-t} M \cong M$, a contradiction. Thus $m \neq 0$. If $m<0$, then there exists some $s \in \mathbb{N}$ such that $\underline{\operatorname{dim}} \tau^{-s t} M=\underline{\operatorname{dim}} M+s m \delta<0$, a contradiction. Hence $m \in \mathbb{N}$.

Remark 3.12. If the assumption that $\tau^{-1} M$ is not a predecessor of any projectives in $\Gamma_{A}$ is dropped or is replaced with the weaker condition that injdim $M \leq 1$, then there is a counter-example, e.g., in the case where $A$ is of type $E_{6}$ and $M=\tau^{-1}\left(A \mathbf{e}_{x_{32}}\right)$. There is also an example for which the smallest value of $m$ is not equal to 1 (see Sect.8.a).

Recall that an $A$-module $M$ is called sincere if $\mathbf{e}_{x} M \neq 0$ for all $x \in Q_{0}$. We say that $M$ is non-sincere if it is not sincere. The following well-known fact follows also from the proposition above (see [11], [4] for general results). 
Corollary 3.13. $A$ is a minimal representation-infinite algebra, i.e., $A / A \mathbf{e}_{x} A$ is representation-finite for all $x \in Q_{0}$.

Proof. It is enough to show that the Auslander-Reiten quiver of $A$ contains only a finite number of non-sincere indecomposable $A$-modules. By the previous proposition it is immediate that the preprojective component contains only a finite number of nonsincere indecomposable $A$-modules. Dually the preinjective component has the same property. In the tubular family any non-sincere indecomposable $A$-module $X$ lies in a non-homogeneous tube $\mathcal{T}$ and has quasi-length less than the rank of $\mathcal{T}$. Thus also there are only a finite number of non-sincere indecomposables in the tubular family.

Proposition 3.14. If an indecomposable A-module $M$ is not sincere, then $\mathbf{u}_{[M]} \in$ $L(A)_{1}$.

Proof. Let $M$ be a non-sincere indecomposable $A$-module, and set $B:=\operatorname{supp} M$. Then by Corollary $3.13, B$ is representation-finite. We regard $\bmod B$ as a full subcategory of $\bmod A$ by the canonical embedding. Then $M$ is regarded as a sincere indecomposable $B$-module, and by the formula (1-2) we see that $L(B)_{1} \subseteq L(A)_{1}$. Therefore it is enough to show that $\mathbf{u}_{[M]} \in L(B)_{1}$. If $B$ is hereditary, then this follows by Lemma 2.9. If $M$ is a preprojective (resp. preinjective) $A$-module, then $M(\infty)=0($ resp. $M(1)=0)$ by Corollary 3.6 because $M$ is not a sincere $A$-module, and then $B$ turns out to be hereditary. Thus in this case the assertion holds. Hence we may assume that $M$ is a regular $A$-module. Since $M$ is not a sincere $A$-module, $M$ is in a non-homogeneous tube of a rank $p>1$ and with quasi-length less than $p$. Thus, in particular, $\underline{\operatorname{dim}} M$ consists of 0 and 1 . We show that $\mathbf{u}_{[M]} \in L(A)_{1}$ by induction on $\operatorname{dim} M$. If $\operatorname{dim} M=1$, then $M$ is simple and the assertion is trivial by definition. Now assume $\operatorname{dim} M>1$. Then the form of $\operatorname{dim} M$ shows that there exists an exact sequence of the form

$$
0 \rightarrow N \rightarrow M \rightarrow S \rightarrow 0 \text { or } 0 \rightarrow S \rightarrow M \rightarrow N \rightarrow 0
$$

with $S$ a simple $A$-module and $N$ an indecomposable $A$-module, and that

$$
\left(F_{S^{K} N^{K}}^{M^{K}}, F_{N^{K} S^{K}}^{M^{K}}\right)=(1,0) \text { or }(0,1),
$$

respectively, for all $K \in \Omega_{A}$. Thus we have $\mathbf{u}_{[M]}= \pm\left[\mathbf{u}_{[S]}, \mathbf{u}_{[N]}\right] \in L(A)_{1}$ because by induction hypothesis both $\mathbf{u}_{[S]}$ and $\mathbf{u}_{[N]}$ are in $L(A)_{1}$.

\section{Realization of simple Lie algebras}

In this section we state our main theorems realizing simple Lie algebras and their root spaces, and give a precise form of Chevalley generators of our realization.

\section{4.a. Main results.}

Definition 4.1. When $\Delta \neq A_{1}$ (resp. $\Delta=A_{1}$ ) we set $I(A)$ to be the ideal of $L(A)_{1}$ (resp. of $L(A)_{1}^{\mathbb{Z}\left[2^{-1}\right]}$ ) generated by the set

$$
\left\{\mathbf{u}_{m\left(e_{x}+\delta\right)}-\mathbf{u}_{m\left(e_{x}\right)} \mid x \in Q_{0}\right\} .
$$

For each $u \in L(A)_{1}^{\mathbb{C}}$ we denote by $\bar{u}$ the coset of $u$ in $L(A)_{1}^{\mathbb{C}} / I(A)^{\mathbb{C}}$. 
Remark 4.2. By Lemmas 5.16, 5.19, and 5.21 that will be proved in the next section we see that $\mathbf{u}_{m\left(e_{x}+\delta\right)} \in L(A)_{1}$ (resp. $\left.\mathbf{u}_{m\left(e_{x}+\delta\right)} \in L(A)_{1}^{\mathbb{Z}\left[2^{-1}\right]}\right)$ for all $x \in Q_{0}$ if $\Delta \neq A_{1}$ (resp. $\Delta=A_{1}$ ).

Notation 4.3. (1) For each $x \in Q_{0}$, the vector $\delta-e_{x}$ is a root of $\chi_{A}$, which enables us to consider the indecomposable $A$-module $T_{x}:=M\left(\delta-e_{x}\right)$. By Lemma 3.14 we have $\mathbf{u}_{\left[T_{x}\right]} \in L(A)_{1}$.

(2) For each $x \in \Delta_{0}$, we set

$$
\varepsilon_{x}:=\overline{\mathbf{u}}_{\left[S_{x}\right]}, \zeta_{x}:=\left\{\begin{array}{ll}
-\overline{\mathbf{u}}_{T_{1}} & \text { if } x=1, \\
\overline{\mathbf{u}}_{T_{x}} & \text { otherwise, }
\end{array} \text { and } \eta_{x}:=\left[\varepsilon_{x}, \zeta_{x}\right] .\right.
$$

Note that all of these are in $L(A)_{1}^{\mathbb{C}} / I(A)^{\mathbb{C}}$.

(3) Let $\left\{E_{x}, F_{x}, H_{x}\right\}_{x \in \Delta_{0}}$ be Chevalley generators of $\mathfrak{g}(\Delta)$, where $\left\{H_{x}\right\}_{x \in \Delta_{0}}$ is a basis of the Cartan subalgebra $\mathfrak{h}$ of $\mathfrak{g}(\Delta),\left\{E_{x}\right\}_{x \in \Delta_{0}}$ (resp. $\left\{F_{x}\right\}_{x \in \Delta_{0}}$ ) are generators of the positive (resp. negative) part $\mathfrak{n}_{+}\left(\right.$resp. $\left.\mathfrak{n}_{-}\right)$of $\mathfrak{g}(\Delta)$.

We are now in a position to state our main theorem.

Theorem 4.4. Let $\Delta$ be a simply-laced Dynkin diagram, $A$ a canonical algebra of type $\Delta$ and $\mathfrak{g}(\Delta)$ the complex simple Lie algebra of type $\Delta$. Further let $\left\{E_{x}, F_{x}, H_{x}\right\}_{x \in \Delta_{0}} \subseteq$ $\mathfrak{g}(\Delta)$ and $\left\{\varepsilon_{x}, \zeta_{x}, \eta_{x}\right\}_{x \in \Delta_{0}} \subseteq L(A)_{1}^{\mathbb{C}} / I(A)^{\mathbb{C}}$ be as in Notation 4.3. Then there is an isomorphism

$$
\phi: \mathfrak{g}(\Delta) \stackrel{\sim}{\rightarrow} L(A)_{1}^{\mathbb{C}} / I(A)^{\mathbb{C}}
$$

such that $\phi\left(E_{x}\right)=\varepsilon_{x}, \phi\left(F_{x}\right)=\zeta_{x}$ and $\phi\left(H_{x}\right)=\eta_{x}$ for all $x \in \Delta_{0}$.

The proof is given in Sect. 6

Definition 4.5. (1) Let $v \in K_{0}(A)$. Then we set $\operatorname{deg} v:=v-v_{\infty} \delta$, which we regard as an element of $K_{0}\left(k Q^{l}\right)$ because $(\operatorname{deg} v)_{\infty}=0$. This defines a linear map $\operatorname{deg}: K_{0}(A) \rightarrow K_{0}\left(k Q^{l}\right), v \mapsto \operatorname{deg} v$. Obviously this is surjective and Ker deg $=\mathbb{Z} \delta$. For an indecomposable $A$-module $M$, we set $\operatorname{deg} M:=\operatorname{deg}(\underline{\operatorname{dim}} M)$ and call it the degree of $M$.

(2) Let $v$ be a positive root of $\chi_{A}$, and $v_{i}\left(i \in Q_{0}\right)$ the smallest entry of $v$. Then we define $\operatorname{org} v:=v-v_{i} \delta$. Since $v \notin \mathbb{Z} \delta$, we have $\operatorname{org} v>0$. Note that $\operatorname{org} v$ is also a positive root of $\chi_{A}$ with $M(\operatorname{org} v)$ non-sincere by Corollary 3.7 and that $\operatorname{org} v=\operatorname{deg} v$ if $\operatorname{rank} v>0$.

(3) We denote by $\operatorname{Rt}\left(\chi_{A}\right)$ (resp. $\operatorname{Rt}_{+}\left(\chi_{A}\right)$ ) the set of all roots (resp. positive roots) of $\chi_{A}$, and by $\operatorname{Rt}(\mathfrak{g}(\Delta))$ (resp. $\operatorname{Rt}_{+}(\mathfrak{g}(\Delta))$ ) the set of all roots (resp. all positive roots) of $\mathfrak{g}(\Delta)$, and set $\mathrm{Rt}_{-}(-):=-\mathrm{Rt}_{+}(-)$. Then $\mathrm{Rt}_{+}\left(\chi_{k Q^{l}}\right)$ is regarded as $\mathrm{Rt}_{+}(\mathfrak{g}(\Delta))$ by identifying $e_{x}$ with $E_{x}$ for all $x \in Q_{0}^{l}=\Delta_{0}$ by Gabriel's Theorem. Thus we have

$$
\operatorname{Rt}(\mathfrak{g}(\Delta))=\operatorname{Rt}_{+}\left(\chi_{k Q^{l}}\right) \cup\left(\operatorname{Rt}_{-}\left(\chi_{k Q^{l}}\right)\right)=\operatorname{Rt}\left(\chi_{k Q^{l}}\right) \subseteq K_{0}\left(k Q^{l}\right) .
$$

Lemma 4.6. The map deg induces a surjective linear map $\operatorname{Rt}_{+}\left(\chi_{A}\right) \rightarrow \operatorname{Rt}(\mathfrak{g}(\Delta))$. In particular, for each indecomposable $A$-module $M$ with $\underline{\operatorname{dim}} M \notin \mathbb{Z} \delta$, $\operatorname{deg} M$ is a root of $\mathfrak{g}(\Delta)$. 
Proof. For each $v \in \operatorname{Rt}_{+}\left(\chi_{A}\right)$ we have $\operatorname{deg} v \in \operatorname{Rt}\left(\chi_{A}\right)$ by Corollary [3.7, and hence $\operatorname{deg} v \in \operatorname{Rt}\left(\chi_{k Q^{l}}\right)=\operatorname{Rt}(\mathfrak{g}(\Delta))$. Therefore $\operatorname{deg}\left(\operatorname{Rt}_{+}\left(\chi_{A}\right)\right) \subseteq \operatorname{Rt}(\mathfrak{g}(\Delta))$. Conversely, for each $w \in \operatorname{Rt}(\mathfrak{g}(\Delta))=\operatorname{Rt}\left(\chi_{k Q^{l}}\right) \subseteq \operatorname{Rt}\left(\chi_{A}\right)$ there exists some $t \in \mathbb{N}$ such that $v:=$ $w+t \delta>0$. Then again by Corollary $3.7 v \in \operatorname{Rt}\left(\chi_{A}\right)$, and hence $v \in \operatorname{Rt}_{+}\left(\chi_{A}\right)$. Here it is obvious that $\operatorname{deg} v=w$. Therefore we have $\operatorname{Rt}(\mathfrak{g}(\Delta)) \subseteq \operatorname{deg}\left(\operatorname{Rt}_{+}\left(\chi_{A}\right)\right)$, and hence $\operatorname{deg}\left(\operatorname{Rt}_{+}\left(\chi_{A}\right)\right)=\operatorname{Rt}(\mathfrak{g}(\Delta))$.

Remark 4.7. In the above, set $M:=M(v)$. Then

(1) If $M$ is preprojective, then $\operatorname{deg} v>0$ by Corollary 3.6 .

(2) If $M$ is preinjective, then $\operatorname{deg} v<0$ by Corollary 3.6.

(3) If $M$ is regular, then $\operatorname{deg} v= \pm \sum_{j=s}^{t} e_{x_{i j}}$ for some $i \in\{1, \ldots, r\}$ and some $s, t$ with $2 \leq s \leq t \leq p(i)$.

For a root $\alpha$ of $\mathfrak{g}(\Delta)$ we denote by $\mathfrak{g}(\Delta)_{\alpha}$ the root space of $\mathfrak{g}(\Delta)$ with root $\alpha$.

Proposition 4.8. Let $v$ be a positive root of $\chi_{A}$. Then

(1) $0 \neq \overline{\mathbf{u}}_{m(v)} \in L(A)_{1}^{\mathbb{C}} / I(A)^{\mathbb{C}}$;

(2) $\phi^{-1}\left(\overline{\mathbf{u}}_{m(v)}\right) \in \mathfrak{g}(\Delta)_{\operatorname{deg} v}$; and

(3) $\mathbb{C} \overline{\mathbf{u}}_{m(v+\delta)}=\mathbb{C} \overline{\mathbf{u}}_{m(v)}$.

The proof is given in Sect.7. This immediately yields the following.

Theorem 4.9. Let $\phi$ be as in Theorem 4.4, $\alpha$ a root of $\mathfrak{g}(\Delta)$ and $v$ a positive root of $\chi_{A}$ with $v-\alpha \in \mathbb{Z} \delta$. Then $\alpha=\operatorname{deg} v$ and the restriction of $\phi$ induces an isomorphism from the root space $\mathfrak{g}(\Delta)_{\alpha}$ to $\mathbb{C} \overline{\mathbf{u}}_{m(v)}$.

4.b. Basis of the Cartan subalgebra. We now give the precise forms of $\eta_{x}$ 's using the list in Proposition 3.3. Noting that $\left[u_{S_{1}},-u_{T_{1}}\right]=\left[u_{T_{1}}, u_{S_{1}}\right]=u_{T_{1}} u_{S_{1}}$ we have

$$
\left[u_{S_{1}},-u_{T_{1}}\right]=\sum_{c \in k} u_{W_{c}}+u_{X_{11}}
$$

in the Lie algebra $\bar{L}(A)_{(q-1)}$. Then in $L(A)_{1}$ we have

$$
\left[\mathbf{u}_{S_{1}},-\mathbf{u}_{T_{1}}\right]=\left(\sum_{c \in K} u_{W_{c}(K)}\right)_{K \in \Omega}+\mathbf{u}_{X_{11}}=: h_{1}
$$

Hence

$$
\eta_{1}= \begin{cases}\overline{\left(\sum_{c \in K^{\times}} u_{W_{c}(K)}\right)_{K \in \Omega}}+\overline{\mathbf{u}}_{X_{11}}+\overline{\mathbf{u}}_{X_{21}} & \text { if } A \text { is of type } A_{n} ; \\ \left.\overline{\left(\sum_{c \in K \backslash\{0,-1\}} u_{W_{c}(K)}\right)}\right)_{K \in \Omega}+\overline{\mathbf{u}}_{X_{11}}+\overline{\mathbf{u}}_{X_{21}}+\overline{\mathbf{u}}_{X_{31}} & \text { otherwise. }\end{cases}
$$

For each $x_{i j} \in \Delta_{0} \backslash\{1\}$ we have

$$
\left[\mathbf{u}_{S_{x_{i j}}}, \mathbf{u}_{T_{x_{i j}}}\right]=\mathbf{u}_{X_{i j}}-\mathbf{u}_{X_{i, j-1}}=: h_{x_{i j}}
$$

in $L(A)_{1}$, and hence

$$
\eta_{x_{i j}}=\bar{h}_{x_{i j}}=\overline{\mathbf{u}}_{X_{i j}}-\overline{\mathbf{u}}_{X_{i, j-1}} .
$$




\section{Preparations of proof of Main Theorem}

5.a. Non-homogeneous tubes. Throughout this section $\mathcal{T}$ is a non-homogeneous tube of rank $p>1$ in $\Gamma_{A}$.

We recall some fundamental facts on nilpotent representations of cyclic quivers ([1]). Consider the cyclic quiver with $p$ vertices

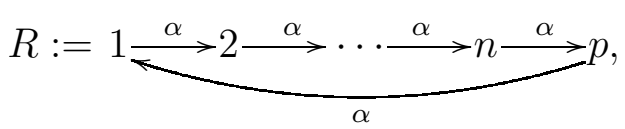

and set $\Lambda$ to be the path-algebra $k R$, which is infinite-dimensional over $k$. Let $J$ be the ideal of $\Lambda$ generated by all arrows. A finite-dimensional $\Lambda$-module $M$ is called nilpotent if $J^{m} M=0$ for some $m \in \mathbb{N}$. Denote by $\bmod _{0} \Lambda\left(\operatorname{resp}\right.$. $\left.\operatorname{ind}_{0} \Lambda\right)$ the full subcategory of $\bmod \Lambda(\operatorname{resp}$. ind $\Lambda)$ consisting of nilpotent $\Lambda$-modules. Then $\bmod _{0} \Lambda$ has almost split sequences, its Auslander-Reiten quiver is isomorphic to $\mathcal{T}$, and $\operatorname{ind}_{0} \Lambda$ is standard, i.e. $\operatorname{ind}_{0} \Lambda \cong k(\mathcal{T})([21,3.6(6)])$. On the other hand $\langle\mathcal{T}\rangle$ is also standard by Theorem 3.4 . Therefore we have

$$
\langle\mathcal{T}\rangle \cong \operatorname{ind}_{0} \Lambda
$$

Of course, if $X \in \mathcal{T}$ is corresponding to $Y \in \operatorname{ind}_{0} \Lambda$ under this isomorphism, then the quasi-length of $X$ is equal to $l(Y)$. In particular, the modules on the mouth of $\mathcal{T}$ are corresponding to simple modules in $\operatorname{ind}_{0} \Lambda$.

Remark 5.1. The shape of $\mathcal{T}$ does not depend on $k$. In particular, $\operatorname{ind}_{0} \Lambda^{K}=\left\{X^{K} \mid X \in\right.$ ind $\left._{0} \Lambda\right\}$ as sets for all $K \in \Omega$.

Lemma 5.2. For each $M \in \operatorname{ind}_{0} \Lambda$, we have $\operatorname{Hom}_{\Lambda}(M, \tau M)=0$ if and only if $l(M)<$ $p$.

Proof. Since $\tau M(i, j)=M(i+1, j)$ for all $(i, j) \in R_{0} \times \mathbb{N}$, where $R_{0}$ is the set of vertices of $R$ (cf. e.g., [3, Proof of Theorem 2.1]), the assertion is easily verified.

Proposition 5.3. Let $X \in \operatorname{ind} A$. Then $X$ is exceptional if and only if

(1) $X$ is preprojective;

(2) $X$ is preinjective; or

(3) $X$ is in a non-homogeneous tube with quasi-length less than the rank of the tube.

Proof. If $X$ is preprojective or preinjective, then $\operatorname{Hom}_{A}(X, \tau X)=0$. Hence by the Auslander-Reiten formula

$$
\operatorname{Ext}_{A}^{1}(X, X) \cong D\left(\overline{\operatorname{Hom}}_{A}(X, \tau X)\right)
$$

(see e.g., [7, Proposition 2.3]) we see that $X$ is exceptional, where for each $M, N \in$ $\bmod A, \overline{\operatorname{Hom}}_{A}(M, N)$ is the factor space of $\operatorname{Hom}_{A}(M, N)$ by the subspace consisting of homomorphisms factoring through an injective module. Now let $X$ be regular, then we have $\operatorname{Ext}_{A}^{1}(X, X) \cong D\left(\operatorname{Hom}_{A}(X, \tau X)\right)$ because $\operatorname{Hom}_{A}(\mathcal{I}, \mathcal{R})=0$. If $X$ is in a homogeneous tube, then $X \cong \tau X$, and hence $X$ cannot be exceptional. Assume that $X$ is in a non-homogeneous tube of rank $p>1$. Then by the lemma above and by (5-1) we have $\operatorname{Hom}_{A}(X, \tau X)=0$ if and only if the quasi-length of $X$ is less than $p$. Hence $X$ is exceptional if and only if the quasi-length of $X$ is less than $p$. 
Notation 5.4. Since $\operatorname{End}_{A}(X)$ is a finite-dimensional local $k$-algebra for each $X \in$ ind $A$, the factor algebra $\operatorname{End}_{A}(X) / \operatorname{rad}_{\operatorname{End}}(X)$ is a field, which we denote by $F_{A}(X)$.

Proposition 5.5. Let $X$ be a module in the non-homogeneous tube $\mathcal{T}$. Then $F_{A}(X)=$ $k$, and hence $X$ is $\Omega$-indecomposable. Thus we may write $\mathbf{u}_{[X]}=\left(u_{\left[X^{K}\right]}\right)_{K \in \Omega}$.

Proof. Let

$$
Y=Y_{1} \rightarrow Y_{2} \rightarrow \cdots \rightarrow Y_{m}=X
$$

be the shortest path in $\mathcal{T}$ with $Y$ an indecomposable module on the mouth of $\mathcal{T}$. Since $Y$ is an exceptional $A$-module by Proposition [5.3, we have $\operatorname{End}_{A}(Y) \cong k$ by Lemma 3.1. Let $\operatorname{rad}_{A}$ be the radical of the category $\bmod A([21$, p. 53]). For $A$-modules $M, N$ set $\operatorname{Irr}(M, N):=\operatorname{rad}_{A}(M, N) / \operatorname{rad}_{A}^{2}(M, N)$ to be the $\left(F_{A}(N), F_{A}(M)\right)$-bimodule of irreducible maps from $M$ to $N$ ([21, p. 55]). Then it follows from the shape of $\mathcal{T}$ that

$$
\operatorname{dim}_{F_{A}\left(Y_{i+1}\right)} \operatorname{Irr}\left(Y_{i}, Y_{i+1}\right)=\operatorname{dim}_{k} \operatorname{Irr}\left(Y_{i}, Y_{i+1}\right)=\operatorname{dim} \operatorname{Irr}\left(Y_{i}, Y_{i+1}\right)_{F_{A}\left(Y_{i}\right)}=1
$$

for all $i \in\{1, \ldots, m-1\}$. This shows that $\mathcal{E}(A) \ni k \cong F_{A}(Y) \cong F_{A}\left(Y_{1}\right) \cong \ldots \cong$ $F_{A}\left(Y_{m}\right) \cong F_{A}\left(X_{t}\right)$.

Now for each $K \in \Omega, \operatorname{End}_{A}\left(X^{K}\right) / \operatorname{rad} \operatorname{End}_{A}\left(X^{K}\right) \cong F_{A}(X)^{K}$ is a field, and hence $X^{K} \in$ ind $A^{K}$.

Definition 5.6. (1) Set $\bar{L}(\mathcal{T}):=\bigoplus_{[X] \in \mathcal{T}} \mathbb{Z} u_{] X]}$. Then $\bar{L}(\mathcal{T}) /(q-1)$ is a Lie subalgebra of the Lie algebra $\bar{L}(A) /(q-1)$ because $\mathcal{T}$ is closed under extensions by [21, 3.1(3)] (or by the fact that $\mathcal{R}$ is separating $\mathcal{P}$ from $\mathcal{I}$ ).

(2) Set $\bar{L}_{0}(\Lambda):=\bigoplus_{[X] \in\left[\operatorname{ind}_{0} \Lambda\right]} \mathbb{Z} u_{[X]}$. Then $\bar{L}_{0}(\Lambda) /(q-1)$ is a Lie algebra with Hall commutator the Lie bracket as in Sect.2.b.

Lemma 5.7. For each $K \in \Omega$ let $\mathcal{T}\left(A^{K}\right)$ be the non-homogeneous tube of $\Gamma_{A^{K}}$ corresponding to $\mathcal{T}$. Then we have

$$
\bar{L}\left(\mathcal{T}\left(A^{K}\right)\right) /(|K|-1) \cong \bar{L}_{0}\left(\Lambda^{K}\right) /(|K|-1) .
$$

Proof. This follows from the isomorphism (5-1) by the formula (1-2).

Definition 5.8. Since $\bmod _{0} \Lambda$ has Hall polynomials by [?, Theorem 2.7], we can define a Lie algebra $\bar{L}_{0}(\Lambda)_{1}:=\bigoplus_{[X] \in\left[\bmod _{0} \Lambda\right]} \mathbb{Z} u_{[X]}$ over $\mathbb{Z}$ with the Lie bracket defined by

$$
\left[u_{[X]}, u_{[Y]}\right]:=\sum_{[Z] \in\left[\operatorname{ind}_{0} \Lambda\right]}\left(\varphi_{[X][Y]}^{[Z]}(1)-\varphi_{[Y][X]}^{[Z]}(1)\right) u_{[Z]}
$$

for all $[X],[Y] \in\left[\operatorname{ind}_{0} \Lambda\right]$ using Hall polynomials $\varphi_{[X][Y]}^{[Z]}$.

Lemma 5.9. We have isomorphisms

$$
\bar{L}\left(\mathcal{T}\left(A^{K}\right)\right) /(|K|-1) \cong \bar{L}_{0}(\Lambda)_{1} /(|K|-1)
$$

for all $K \in \Omega$. 
Proof. Since $F_{\left[X^{K}\right]\left[Y^{K}\right]}^{\left[Z^{K}\right]}=\varphi_{[X][Y]}^{[Z]}(1)$ in $\mathbb{Z} /(|K|-1) \mathbb{Z}$ for all $X, Y, Z \in \operatorname{ind}_{0} \Lambda$ and all $K \in \Omega$, we have $\bar{L}_{0}\left(\Lambda^{K}\right) /(|K|-1) \cong \bar{L}_{0}(\Lambda)_{1} /(|K|-1)$ (here notations $F_{\left[X^{K}\right]\left[Y^{K}\right]}^{\left[Z^{K}\right]}$ are for $\Lambda$-modules) by Remark 5.1. Hence the assertion follows by Lemma 5.7.

The Lie bracket of $\bar{L}_{0}(\Lambda)_{1}$ is easily described as follows. First we have a bijection $\left[\operatorname{ind}_{0} \Lambda\right] \rightarrow R_{0} \times \mathbb{N}$ defined by $[M] \mapsto(i, j)$ with top $M \cong S_{i}$ and $l(M)=j$ because all modules in $\operatorname{ind}_{0} \Lambda$ are uniserial. We identify $R_{0}$ with $\mathbb{Z} / \mathbb{Z} p$, and for each $(i, j) \in R_{0} \times \mathbb{N}$ denote by $m(i, j)$ the isoclass of the indecomposable modules in $\operatorname{ind}_{0} \Lambda$ corresponding to $(i, j)$. We choose a representative $M(i, j) \in m(i, j)$ for all $(i, j) \in R_{0} \times \mathbb{N}$. Then as calculated in [1, 1.2] we have the following: For each $m(i, j), m(f, g) \in\left[\operatorname{ind}_{0} \Lambda\right]$

in $\bar{L}_{0}(\Lambda)_{1}$.

$$
\left[u_{m(i, j)}, u_{m(f, g)}\right]=\delta_{(i+j), f} u_{m(i, j+g)}-\delta_{(f+g), i} u_{m(f, j+g)}
$$

Definition 5.10. Define $L_{0}(\Lambda)_{1}$ to be the Lie subalgebra of $\bar{L}_{0}(\Lambda)_{1}$ generated by all $u_{[S]}$ with $S$ simple modules in $\operatorname{ind}_{0} \Lambda$.

From the formula (5-3) we obtain the following.

Lemma 5.11. Let $X \in \operatorname{ind}_{0} \Lambda$ and assume that $l:=l(X) \notin \mathbb{Z} p$. If $p>2$ (resp. if $p=2$ ), then $u_{[X]}$ (resp. $2^{m} u_{[X]}$ with $\left.m:=(l-1) / 2\right)$ is obtained from $\left\{u_{[S]} \mid\right.$ $S$ simple in $\left.\operatorname{ind}_{0} \Lambda\right\}$ by Lie brackets in $\bar{L}_{0}(\Lambda)_{1}$, in particular, we have $u_{[X]} \in L_{0}(\Lambda)_{1}^{\mathbb{Q}}$.

Proof. We put $L$ to be the subset of $\bar{L}_{0}(\Lambda)_{1}$ consisting of elements obtained from $u_{[S]}$ with $S$ simple modules in $\operatorname{ind}_{0} \Lambda$ by Lie brackets in $\bar{L}_{0}(\Lambda)_{1}$. There exist a unique $m \in \mathbb{N}_{0}$ and a unique $r \in\{1, \ldots, p-1\}$ such that $l=m p+r$. We show the assertion by induction on $l \geq 1$. If $l=1$, then $X$ is simple and there is nothing to show. Assume $l>1$. Without loss of generality we may assume that $X=M(1, l)$.

Case 1. $p>2, r>1$. In this case if we put $t:=(m-1) p+(r-1)$, then $l=t+(p+1), t, p+1<l$, and $t, p+1 \notin \mathbb{Z} p$, which implies $u_{m(1, t)}, u_{m(r, p+1)} \in L$ by induction hypothesis. Since $r+1 \neq 1$ in $\mathbb{Z} / \mathbb{Z} p$, we have $\left[u_{m(1, t)}, u_{m(r, p+1)}\right]=u_{[X]}$ by the formula (5-3). Hence $u_{[X]} \in L$.

Case 2. $p>2, r=1$. In this case if we put $t:=(m-1) p+(p-1)$, then $l=t+2$, $t, 2<l$, and $t, 2 \notin \mathbb{Z} p$, which implies $u_{m(t, t)}, u_{m(p, 2)} \in L$ by induction hypothesis. Since $1 \neq 2$ in $\mathbb{Z} / \mathbb{Z} p$ we have $\left[u_{m(1, t)}, u_{m(p, 2)}\right]=u_{[X]}$ by the formula (5-3). Hence $u_{[X]} \in L$.

Case 3. $p=2$. In this case by induction hypothesis we have $2^{m-1} u_{m(1, l-2)} \in L$. Then $\left[\left[u_{m(1,1)}, u_{m(2,1)}\right], u_{m(1, l-2)}\right]=\left[u_{m(1,2)}-u_{m(2,2)}, u_{m(1, l-2)}\right]=2 u_{[X]}$ shows that $2^{m} u_{[X]} \in$ $L$.

By (5-1) and (5-2) the lemma above implies the following:

Proposition 5.12. Let $X \in \mathcal{T}$ and assume that the quasi-length $l$ of $X$ is not a multiple of $p$. If $p>2$ (resp. if $p=2$ ), then $\mathbf{u}_{[X]}$ (resp. $2^{m} \mathbf{u}_{[X]}$ with $\left.m:=(l-1) / 2\right)$ is obtained from $\left\{\mathbf{u}_{[Y]} \mid Y\right.$ modules on the mouth of $\left.\mathcal{T}\right\}$ by Lie brackets in the Lie algebra П. In particular, $\mathbf{u}_{[X]} \in L(A)_{1}^{\mathbb{Q}}$ by Lemma 3.14. 
Remark 5.13. In other words, the last statement above is stated as follows: Let $v$ be a positive root of $\chi_{A}$. If $M(v)$ is regular, then $\mathbf{u}_{m(v)} \in L(A)_{1}^{\mathbb{Q}}$. In particular, if $M(v)$ is exceptional, then $\mathbf{u}_{m(v)} \in L(A)_{1}$. The last statement also follows by Proposition 3.14 because in this case $M(v)$ is not sincere.

The following is obvious by the formula (5-3):

Lemma 5.14. In $\bar{L}_{0}(\Lambda)_{1}$ we have $\left[u_{m(i, s p)}, u_{m(j, t p)}\right]=0$ for all $i, j \in R_{0}$ and for all $s, t \in \mathbb{N}$.

Proposition 5.15. Let $\mathcal{T}$ be a non-homogeneous tube of $\Gamma_{A}$, and let $X, Y \in \mathcal{T}$. If both $\underline{\operatorname{dim}} X$ and $\underline{\operatorname{dim}} Y$ are in $\mathbb{Z} \delta$, then $\left[\mathbf{u}_{[X]}, \mathbf{u}_{[Y]}\right]=0$ in $\Pi$.

Proof. Both the quasi-lengths of $X$ and $Y$ are multiples of $p$ by Sect. 3.g. Hence the assertion follows from the lemma above by (5-2).

\section{5.b. Regular root modules modulo $\delta$.}

Lemma 5.16. Let $v$ be a positive root of $\chi_{A}$ with $M(v)$ a regular module. Then there exists an $x \in Q_{0} \backslash\{1, \infty\}$ which is determined by $v$ modulo $\mathbb{Z} \delta$ such that $\left[\mathbf{u}_{m(v)}, h_{x}\right]=$ $\mathbf{u}_{m(v+\delta)}$ in $\Pi$.

Proof. Set $M:=M(v)$. Then $M$ is in a non-homogeneous tube $\mathcal{T}$ of rank $p>1$. Let $\left\{S_{i} \mid i \in \mathbb{Z} / p \mathbb{Z}\right\}$ be the set of quasi-simples in $\mathcal{T}$ with $S_{i+1}=\tau S_{i}(i \in \mathbb{Z} / p \mathbb{Z})$. Then $M=S_{i}[t]$ for some $i \in \mathbb{Z} / p \mathbb{Z}$ and $t \in \mathbb{N}$ with $p \nmid t$. Express $t$ as $t=m p+r$ with $m \geq 0$ and $1 \leq r \leq p-1$. Then $\mathbf{u}_{\left[S_{i+r}[p]\right]}-\mathbf{u}_{\left[S_{i+r+1}[p]\right]}=h_{x}$ for a unique $x \in Q_{o} \backslash\{1, \infty\}(x$ is determined by $i+r$ ) and we have

$$
\left[\mathbf{u}_{m(v)}, h_{x}\right]=\left[\mathbf{u}_{m(v)}, \mathbf{u}_{\left[S_{i+r}[p]\right]}-\mathbf{u}_{\left[S_{i+r+1}[p]\right]}\right]=\mathbf{u}_{\left[S_{i}[t+p]\right]}=\mathbf{u}_{m(v+\delta)} .
$$

Next we show that the vertex $x \in Q_{0}$ is determined by $v$ modulo $\mathbb{Z} \delta$. Let $v^{\prime}$ be another positive root of $\chi_{A}$ with $M\left(v^{\prime}\right)$ regular and assume that $v-v^{\prime} \in \mathbb{Z} \delta$. Then $M\left(\delta^{\prime}\right)$ is also in $\mathcal{T}$ and $M\left(\delta^{\prime}\right)=S_{i^{\prime}}\left[t^{\prime}\right]$ for some $i^{\prime} \in \mathbb{Z} / p \mathbb{Z}$ and $t^{\prime} \in \mathbb{N}$ with $p \nmid t^{\prime}$. Let $t^{\prime}=m^{\prime} p+r^{\prime}$ with $m^{\prime} \geq 0$ and $1 \leq r^{\prime} \leq p-1$. Then since $v-v^{\prime} \in \mathbb{Z} \delta$, we have $i=i^{\prime}$ and $t-t^{\prime} \in p \mathbb{Z}$. Hence $r=r^{\prime}$ and thus $i+r=i^{\prime}+r^{\prime}$ as desired.

Remark 5.17. Let $v$ be a positive root of $\chi_{A}$ with $M(v)$ a regular module. In this case $v^{\prime}:=\operatorname{org} v$ (see Definition 4.5) is the unique positive root of $\chi_{A}$ such that $M\left(v^{\prime}\right)$ exceptional and $v-v^{\prime} \in \mathbb{Z} \delta$. As easily seen it is given by

$$
\operatorname{org} v= \begin{cases}\operatorname{deg} v & \text { if } \operatorname{deg} v>0 \\ \operatorname{deg} v+\delta & \text { otherwise. }\end{cases}
$$

Proposition 5.18. Let $v$ be a positive root of $\chi_{A}$ with $M(v)$ a regular module. Then $\mathbf{u}_{m(v)}-\mathbf{u}_{m(\operatorname{org} v)} \in I(A)$.

Proof. Set $v^{\prime}:=\operatorname{org} v$ and let $m$ be the non-negative integer such that $v=v^{\prime}+m \delta$. It is enough to show that

$$
\mathbf{u}_{m(v-t \delta)}-\mathbf{u}_{m(v-(t+1) \delta)} \in I(A)
$$

for all $t=0,1, \ldots, m-1$. By Lemma 5.16 we have

$$
\mathbf{u}_{m(v-t \delta)}-\mathbf{u}_{m(v-(t+1) \delta)}=\left[\mathbf{u}_{m(v-(t+1) \delta)}-\mathbf{u}_{m(v-(t+2) \delta)}, h_{x}\right]
$$


for all $t=0,1, \ldots, m-2$. Hence it is enough to show that

$$
\mathbf{u}_{m\left(v^{\prime}+\delta\right)}-\mathbf{u}_{m\left(v^{\prime}\right)} \in I(A) .
$$

Let $\mathcal{T}$ be the non-homogeneous tube containing $M\left(v^{\prime}\right)$. Then the set of quasi-simple modules in $\mathcal{T}$ is equal to $\mathcal{S}:=\left\{M\left(e_{i 2}\right), M\left(e_{i 3}\right), \ldots, M\left(e_{i p(i)}\right), M\left(v_{i}\right)\right\}$ for some $i \in$ $\{1,2,3\}$, where $v_{i}:=\delta-\sum_{j=2}^{p(i)} e_{i j}$. Set $p:=p(i), e_{j}:=e_{i j} \quad(j=2, \ldots, p)$ for short.

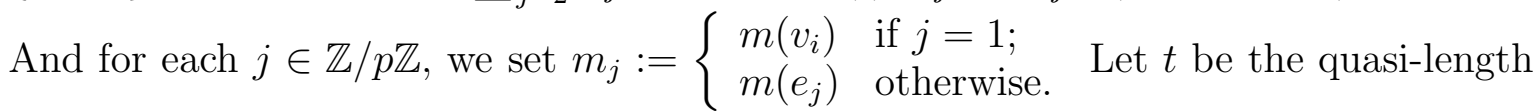
of $M\left(v^{\prime}\right)$. Then $1 \leq t \leq p-1$ and there exists some $j \in \mathbb{Z} / p \mathbb{Z}$ such that

$$
\mathbf{u}_{m\left(v^{\prime}\right)}=\left[\mathbf{u}_{m_{j}}, \mathbf{u}_{m_{j+1}}, \ldots, \mathbf{u}_{m_{j+t-1}}\right] .
$$

Case (i). If $\{j, j+1, \ldots, j+t-1\} \neq\{1\}$, then there exists some $s \in\{j, j+1, \ldots, j+$ $t-1\} \backslash\{1\}$ such that

$$
\mathbf{u}_{m\left(v^{\prime}+\delta\right)}=\left[\mathbf{u}_{m_{j}}, \ldots, \mathbf{u}_{m_{s-1}}, \mathbf{u}_{m\left(e_{s}+\delta\right)}, \mathbf{u}_{m_{s+1}}, \ldots, \mathbf{u}_{m_{j+t-1}}\right] .
$$

Hence we have

$$
\mathbf{u}_{m\left(v^{\prime}+\delta\right)}-\mathbf{u}_{m\left(v^{\prime}\right)}=\left[\mathbf{u}_{m_{j}}, \ldots, \mathbf{u}_{m_{s-1}}, \mathbf{u}_{m\left(e_{s}+\delta\right)}-\mathbf{u}_{m\left(e_{s}\right)}, \mathbf{u}_{m_{s+1}}, \ldots, \mathbf{u}_{m_{j+t-1}}\right],
$$

which is in $I(A)$ because $\mathbf{u}_{m\left(e_{s}+\delta\right)}-\mathbf{u}_{m\left(e_{s}\right)} \in I(A)$.

Case (ii). Otherwise, we have $v^{\prime}=v_{i}$. Then $\mathbf{u}_{m\left(v^{\prime}\right)}=\left[\mathbf{u}_{m\left(v_{i}-e_{1}\right)}, \mathbf{u}_{m\left(e_{1}\right)}\right]$ and $\mathbf{u}_{m\left(v^{\prime}+\delta\right)}=\left[\mathbf{u}_{m\left(v_{i}-e_{1}\right)}, \mathbf{u}_{m\left(e_{1}+\delta\right)}\right]$. Since $\mathbf{u}_{m\left(v_{i}-e_{1}\right)} \in L(A)_{1}$ and $\mathbf{u}_{m\left(e_{1}+\delta\right)}-\mathbf{u}_{m\left(e_{1}\right)} \in I(A)$ we have

$$
\mathbf{u}_{m\left(v^{\prime}+\delta\right)}-\mathbf{u}_{m\left(v^{\prime}\right)}=\left[\mathbf{u}_{m\left(v_{i}-e_{1}\right)}, \mathbf{u}_{m\left(e_{1}+\delta\right)}-\mathbf{u}_{m\left(e_{1}\right)}\right] \in I(A) .
$$

\section{5.c. Simple modules modulo $\delta$.}

Lemma 5.19. Assume that $\Delta \neq A_{1}$. Then for each $t \in \mathbb{N}_{0}$ we have the following.

(1) $\left[\mathbf{u}_{m\left(e_{1}+t \delta\right)}, h_{12}\right]=\mathbf{u}_{m\left(e_{1}+(t+1) \delta\right)}$; and

(2) $\left[\mathbf{u}_{m\left(e_{\infty}+t \delta\right)}, h_{1 p(1)}\right]=\mathbf{u}_{m\left(e_{\infty}+(t+1) \delta\right)}$.

Proof. We only show the statement (2), the first one is shown similarly. Now $h_{1 p(1)}=$ $\mathbf{u}_{\left[X_{1 p(1)}\right]}-\mathbf{u}_{\left[X_{1, p(1)-1}\right]} \in L(A)_{1}$, and hence it is enough to show the following two equalities:

$$
\begin{aligned}
{\left[\mathbf{u}_{m\left(e_{\infty}+t \delta\right)}, \mathbf{u}_{\left[X_{1 p(1)}\right]}\right] } & =\mathbf{u}_{m\left(e_{\infty}+(t+1) \delta\right)} ; \\
{\left[\mathbf{u}_{m\left(e_{\infty}+t \delta\right)}, \mathbf{u}_{\left[X_{1, p(1)-1}\right]}\right] } & =0 .
\end{aligned}
$$

The equality (5-4) holds if and only if for all $K \in \Omega$, we have

$$
\left[u_{\left[M\left(e_{\infty}+t \delta\right)^{K}\right]}, u_{\left[X_{1 p(1)}^{K}\right]}\right] \equiv u_{\left[M\left(e_{\infty}+(t+1) \delta\right)^{K}\right]} \quad(\bmod (|K|-1)) .
$$

Since $F_{X_{1, p I 1)}^{K}, M\left(e_{\infty}+t \delta\right)^{K}}^{M\left(e_{\infty}+(t+1) \delta\right)^{K}}=0$, this holds if $F_{M\left(e_{\infty}+t \delta\right)^{K}, X_{1, p(1)}^{K}}^{M\left(e_{\infty}+(t+1) \delta\right)^{K}}=1$. To this end we only have to show the following:

$$
F_{M\left(e_{\infty}+t \delta\right), X_{1, p(1)}}^{M\left(e_{\infty}+(t+1) \delta\right)}=1
$$


because the value of the right hand side does not depend on $q$. Similarly the equality (5-5) holds if the following holds:

$$
F_{M\left(e_{\infty}+t \delta\right), X_{1, p(1)-1}}^{M\left(e_{\infty}+(t+1) \delta\right)}=0 .
$$

First we show (5-7). Let $f \in \operatorname{Hom}_{A}\left(X_{1, p(1)-1}, M\left(e_{\infty}+(t+1) \delta\right)\right)$. Then we have a commutative diagram

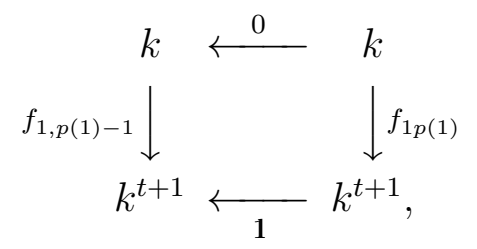

which shows that $f_{1, p(1)}=0$, and that $f$ is not a monomorphism. Therefore there is no exact sequence of the form

$$
0 \rightarrow X_{1, p(1)-1} \rightarrow M\left(e_{\infty}+(t+1) \delta\right) \rightarrow M\left(e_{\infty}+t \delta\right) \rightarrow 0,
$$

which proves the equality (5-7). Next we show the equality (5-6). Assume that we have an exact sequence

$$
0 \longrightarrow X_{1, p(1)} \stackrel{f}{\longrightarrow} M\left(e_{\infty}+(t+1) \delta\right) \stackrel{g}{\longrightarrow} M\left(e_{\infty}+t \delta\right) \longrightarrow 0 .
$$

Then the direct calculation shows that

$$
\begin{aligned}
& f_{\infty}=\left[\begin{array}{c}
0 \\
\vdots \\
0 \\
a
\end{array}\right] \in \operatorname{Mat}_{(t+2) \times 1}(k), \quad g_{\infty}=\left[\begin{array}{cccc} 
& & & 0 \\
& g_{1} & & \vdots \\
& & & 0 \\
* & \cdots & * & c
\end{array}\right] \in \operatorname{Mat}_{(t+1) \times(t+2)}(k) \\
& f_{1}=\left[\begin{array}{c}
0 \\
\vdots \\
0 \\
a
\end{array}\right] \in \operatorname{Mat}_{(t+1) \times 1}(k), \quad g_{\infty}=\left[\begin{array}{cccc}
b & * & \cdots & * \\
0 & & \\
\vdots & & g_{1} \\
0 &
\end{array}\right] \in \operatorname{Mat}_{(t+1) \times(t+2)}(k)
\end{aligned}
$$

for some $a, b, c \in k$. Therefore we have

$$
g_{\infty}=\left[\begin{array}{cccc}
b & c & & 0 \\
& b & \ddots & \\
& & \ddots & c \\
0 & & & b
\end{array}\right] \in \operatorname{Mat}_{(t+1) \times(t+2)}(k), \quad g_{1}=\left[\begin{array}{cccc}
b & c & & 0 \\
& b & \ddots & \\
& & \ddots & c \\
0 & & & b
\end{array}\right] \in \operatorname{Mat}_{t \times(t+1)}(k) .
$$

Then $f$ is a monomorphism, $g$ is an epimorphism and $g f=0$ if and only if $a \neq 0, c=0$ and $b \neq 0$. Hence we have $\left|W_{M\left(e_{\infty}+t \delta\right), X_{1 p(1)}}^{M\left(e_{\infty}+(t+1) \delta\right)}\right|=(q-1)^{2}$, which shows the equality (5-6) (see Sect.1.b).

To deal with the case that $\Delta=A_{1}$ we need the following formula: 
Lemma 5.20. Assume that $\Delta=A_{1}$. Let $K \in \Omega, l, m \in \mathbb{N}_{0}$ and $X \in$ ind $A^{K}$. Then we have

$$
F_{X, M\left(e_{1}+l \delta\right)^{K}}^{M\left(e_{1}+m \delta\right)^{K}}=F_{M\left(e_{\infty}+l \delta\right)^{K}, X}^{M\left(e_{\infty}+m \delta\right)^{K}}= \begin{cases}1 & \text { if } l<m, \underline{\operatorname{dim}} X=(m-l) \delta \\ 0 & \text { otherwise }\end{cases}
$$

Proof. This follows from a direct calculation, or from Szántó [29, Lemma 1.3].

Using the lemma above we obtain the statement corresponding to Lemma 5.19 in the $A_{1}$ case.

Lemma 5.21. Assume that $\Delta=A_{1}$. Then for each $t \in \mathbb{N}_{0}$ we have the following.

(1) $\left[h_{1}, \mathbf{u}_{m\left(e_{1}+t \delta\right)}\right]=2 \mathbf{u}_{m\left(e_{1}+(t+1) \delta\right)}$; and

(2) $\left[\mathbf{u}_{m\left(e_{\infty}+t \delta\right)}, h_{1}\right]=2 \mathbf{u}_{m\left(e_{\infty}+(t+1) \delta\right)}$.

Proof. (1) It is enough to show that $\left[\sum_{c \in K} u_{W_{c}(K)}+u_{X_{11}}, u_{m\left(e_{1}+t \delta\right)^{K}}\right]=2 u_{m\left(e_{1}+(t+1) \delta\right)^{K}}$ in $\bar{L}(A)_{1} /(|K|-1)$ for each $K \in \Omega$. By Lemma 5.20 the left hand side is equal to $(|K|+1) u_{m\left(e_{1}+(t+1) \delta\right)^{K}}$, which is equal to the right hand side in $\bar{L}(A)_{1} /(|K|-1)$.

(2) This is shown similarly.

Proposition 5.22. For each $x \in Q_{0}$ and $t \in \mathbb{N}$, we have

$$
\mathbf{u}_{m\left(e_{x}+t \delta\right)}-\mathbf{u}_{m\left(e_{x}\right)} \in \begin{cases}I(A) & \text { if } \Delta \neq A_{1} \\ I(A)^{\mathbb{Z}\left[2^{-1}\right]} & \text { if } \Delta=A_{1}\end{cases}
$$

Proof. If $x \neq 1, \infty$, then $v=e_{x}+t \delta$ is a positive root with $M(v)$ a regular module and $\operatorname{deg} v=e_{x}$, and hence the statement holds by Proposition 5.18. Now let $x=1, \infty$. First we consider the case that $\Delta \neq A_{1}$. It is enough to show that $\mathbf{u}_{m\left(e_{x}+(t+1) \delta\right)}-$ $\mathbf{u}_{m\left(e_{x}+t \delta\right)} \in I(A)$ by induction on $t \in \mathbb{N}_{0}$. This holds for $t=0$ by definition of $I(A)$. Let $t \geq 1$. By setting $u:=\left\{\begin{array}{ll}h_{12} & \text { if } x=1 \\ h_{1 p(1)} & \text { if } x=\infty\end{array}\right.$ we have $\mathbf{u}_{m\left(e_{x}+(t+1) \delta\right)}-\mathbf{u}_{m\left(e_{x}+t \delta\right)}=$ $\left[\mathbf{u}_{m\left(e_{x}+t \delta\right)}-\mathbf{u}_{m\left(e_{x}+(t-1) \delta\right)}, u\right]$ by Lemma 5.19. Since $u \in L(A)_{1}$, the right hand side is in $I(A)$ by induction hypothesis. Next consider the case that $\Delta=A_{1}$. Then by setting $u:=\left\{\begin{array}{ll}-h_{1} & \text { if } x=1 \\ h_{1} & \text { if } x=\infty\end{array}\right.$ we have $2\left(\mathbf{u}_{m\left(e_{x}+(t+1) \delta\right)}-\mathbf{u}_{m\left(e_{x}+t \delta\right)}\right)=\left[\mathbf{u}_{m\left(e_{x}+t \delta\right)}-\mathbf{u}_{m\left(e_{x}+(t-1) \delta\right)}, u\right]$ for each $t \geq 1$ by Lemma 5.21, This proves the remaining statement.

5.d. Stability of Hall numbers. Let $K \in \Omega$ and $X, Y \in \operatorname{ind} A^{K}$. If $v:=\underline{\operatorname{dim}} X+$ $\underline{\operatorname{dim}} Y$ is a root of $\chi$, then we set

$$
b_{[X],[Y]}:=\left(F_{X Y}^{M(v)^{K}}-F_{Y X}^{M(v)^{K}}\right)+(|K|-1) \mathbb{Z} \in \mathbb{Z} /(|K|-1) \mathbb{Z} .
$$

Note that in this case we have $\left[u_{[X]}, u_{[Y]}\right]=b_{[X],[Y]} u_{m(v)^{K}} \in \bar{L}\left(A^{K}\right) /(|K|-1)$ and $b_{[X],[Y]}$ is uniquely determined by this property.

Proposition 5.23. Assume that $\Delta \neq A_{1}$. Let $K \in \Omega, X \in$ ind $A^{K}$, and $x \in Q_{0}$. If $\underline{\operatorname{dim}} X \in \mathbb{Z} \delta$, then for any $t \in \mathbb{N}$ we have

$$
b_{[X], m\left(e_{x}+t \delta\right)^{K}}=b_{[X], m\left(e_{x}\right)^{K}} .
$$


Proof. We have $\underline{\operatorname{dim}} X=d \delta$ for some $d \in \mathbb{N}$. By Lemma 5.16 and Lemma 5.19 there exists some $x_{i j} \in Q_{0} \backslash\{1, \infty\}$ such that $u:=u_{X_{i j}^{K}}-u_{X_{i, j-1}^{K}}$ satisfies $u_{m\left(e_{x}+t \delta\right)^{K}}=$

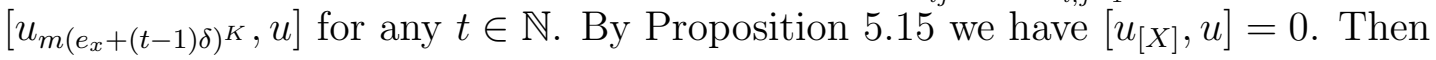

$$
\begin{aligned}
& {\left[u_{[X]}, u_{m\left(e_{x}+t \delta\right)^{K}}\right]=\left[u_{[X]},\left[u_{m\left(e_{x}+(t-1) \delta\right)^{K}}, u\right]\right]} \\
& =\left[\left[u_{[X]}, u_{m\left(e_{x}+(t-1) \delta\right)^{K}}\right], u\right]+\left[u_{m\left(e_{x}+(t-1) \delta\right)^{K}},\left[u_{[X]}, u\right]\right] \\
& =b_{[X], m\left(e_{x}+(t-1) \delta\right)^{K}}\left[u_{\left.m\left(e_{x}+(t-1+d) \delta\right)^{K}, u\right]}\right. \\
& =b_{[X], m\left(e_{x}+(t-1) \delta\right)^{K}} u_{m\left(e_{x}+(t+d) \delta\right)^{K}},
\end{aligned}
$$

which shows $b_{[X], m\left(e_{x}+t \delta\right)^{K}}=b_{[X], m\left(e_{x}+(t-1) \delta\right)^{K}}$. By repeating this we obtain the assertion.

When $\Delta=A_{1}$, we have the following by Lemma 5.20 :

Proposition 5.24. Assume that $\Delta=A_{1}$. Let $m \in \mathbb{N}, K \in \Omega$, and $X \in \operatorname{ind} A^{K}$. If $\underline{\operatorname{dim}} X=m \delta$, then for each $x \in Q_{0}=\{1, \infty\}$ and each $t \in \mathbb{N}$ we have $F_{X, M\left(e_{x}\right)^{K}}^{M\left(e_{x}+m \delta\right.}=$ $F_{X, M\left(e_{x}+t \delta\right)^{K}}^{M\left(e_{x}+(t+m) \delta\right.}(=1)$. Thus

$$
b_{[X], m\left(e_{x}+t \delta\right)^{K}}=b_{[X], m\left(e_{x}\right)^{K}} .
$$

\section{5.e. Hall polynomials.}

Lemma 5.25. If $M$ and $N$ are preprojective A-modules, then there exists polynomials $\varphi_{*, N}^{M}(T), \varphi_{N, *}^{M} \in \mathbb{Z}[T]$ such that

$$
F_{*, N^{K}}^{M^{K}}=\varphi_{*, N}^{M}(|K|) \text { and } F_{N^{K}, *}^{M^{K}}=\varphi_{N, *}^{M}(|K|)
$$

for all $K \in \Omega$.

Proof. Since the class of preprojective $A$-modules are closed under submodules, the images of homomorphisms between preprojective $A$-modules are again preprojective. Noting this the statement can be shown by the same argument as in the step (4) of the proof of [23, Theorem 1] if we replace the whole Auslander-Reiten quiver of $A$ by its preprojective component. The details are left to the reader.

Proposition 5.26. If $M$ and $N$ are preprojective $A$-modules and $S$ is a simple $A$ module, then there exist Hall polynomials $\varphi_{S N}^{M}, \varphi_{N S}^{M}$.

Proof. If $\underline{\operatorname{dim}} M-\underline{\operatorname{dim}} N \neq \underline{\operatorname{dim}} S$, then we can take $\varphi_{S N}^{M}=0, \varphi_{N S}^{M}=0$. Assume that $\underline{\operatorname{dim}} M-\underline{\operatorname{dim}} N=\underline{\operatorname{dim}} S$. Then since any $A$-module having dimension vector $\underline{\operatorname{dim}} S$ is isomorphic to $S$, we can take $\varphi_{S N}^{M}=\varphi_{* N}^{M}$ and $\varphi_{N S}^{M}=\varphi_{N *}^{M}$ by using Lemma 5.25,

Recall that an algebra is called representation-directed if it is representation-finite and its Auslander-Reiten quiver does not contain oriented cycles ([23]). By Corollary 3.13 an $A$-module $X$ is non-sincere if and only if supp $X$ is representation-directed because the latter is equivalent to saying that $\operatorname{supp} X$ is representation-finite in our case. Note that regular exceptional $A$-modules are non-sincere, for which we apply the following later. 
Proposition 5.27. Let $M$ and $N$ be preprojective A-modules. If $X$ is a non-sincere $A$-module, then there exists a Hall polynomial $\varphi_{X N}^{M}$.

Proof. Since $X$ is non-sincere, $\operatorname{supp} X$ is representation-directed as explained above. Noting that any $A$-module with dimension vector $\underline{\operatorname{dim}} X$ is a module over $\operatorname{supp} X$, the assertion can be shown by the same argument as in the last step of the proof of [23, Theorem 1]. For the benefit of the reader we outline the proof.

If $\underline{\operatorname{dim}} M \neq \underline{\operatorname{dim}} X+\underline{\operatorname{dim}} N$, then we can take $\varphi_{X N}^{M}=0$. Therefore we may assume that $\underline{\operatorname{dim}} M=\underline{\operatorname{dim}} X+\underline{\operatorname{dim}} N$. We define $\varphi_{X N}^{M}$ by induction on $\underline{\operatorname{dim}} X \in K_{0}(A)$ (here $K_{0}(A)$ is regarded as a poset by the order defined in Definition [1.5). If $\underline{\operatorname{dim}} X=0$, then we may take $\varphi_{X N}^{M}=1$. Assume $\underline{\operatorname{dim}} X \neq 0$. Let $X=\bigoplus_{i=1}^{m} X_{i}^{\left(t_{i}\right)}$ be a direct sum decomposition of $X$ into pairwise non-isomorphic indecomposable $A$-modules $X_{i}$. If $m=1, X$ is called homogeneous. Note that all $X_{i}$ are modules over $\operatorname{supp} X$ and regarded as vertices in the AR-quiver $\Gamma$ of $\operatorname{supp} X$. Since $\operatorname{supp} X$ is representationdirected, we can regard the set $\Gamma_{0}$ of vertices of $\Gamma$ as a poset by defining an order as follows: For $x, y \in \Gamma, x$ is smaller than $y$ if and only if there exists a path from $x$ to $y$ in $\Gamma$. We may assume that $X_{1}$ is minimal among all $X_{i}$ in $\Gamma_{0}$.

Case 1. $X$ is non-homogeneous (i.e., $m>1$ ). Let $X^{\prime} ;=X_{1}^{\left(t_{1}\right)}$ and $X^{\prime \prime}:=\bigoplus_{i \neq 1} X_{i}^{\left(t_{i}\right)}$. Then $X=X^{\prime} \oplus X^{\prime \prime}, \operatorname{Hom}_{A}\left(X^{\prime \prime}, X^{\prime}\right)=0$ and $\operatorname{Ext}_{A}^{1}\left(X^{\prime}, X^{\prime \prime}\right)=0$. We define $\varphi_{X N}^{M}$ as follows.

$$
\varphi_{X N}^{M}:=\sum_{V \in \mathcal{V}} \varphi_{X^{\prime} V}^{M} \varphi_{X^{\prime \prime} N}^{V}
$$

where $\mathcal{V}$ is a complete set of representatives of isoclasses of submodules $V$ of $M$ such that $\underline{\operatorname{dim}} V=\underline{\operatorname{dim}} X^{\prime \prime}+\underline{\operatorname{dim}} N$. Then all the terms on the right hand side are already defined by the induction hypothesis because $N, V, M$ are preprojective and $X^{\prime}, X^{\prime \prime}$ are modules over $\operatorname{supp} X$. Here we can show that $\varphi_{X N}^{M}$ is a Hall polynomial, i.e., that $\varphi_{X N}^{M}(|K|)=F_{X^{K} N^{K}}^{M^{K}}$ for all $K \in \Omega$, by using the associativity of Hall multiplication and the facts that $\operatorname{Hom}_{A}\left(X^{\prime \prime}, X^{\prime}\right)=0$ and $\operatorname{Ext}_{A}^{1}\left(X^{\prime}, X^{\prime \prime}\right)=0$.

Case 2. $X$ is homogeneous. In this case we define $\varphi_{X N}^{M}$ as follows.

$$
\varphi_{X N}^{M}:=\varphi_{*, N}^{M}-\sum_{U \in \mathcal{U}} \varphi_{U N}^{M}
$$

where $\varphi_{*, N}^{M}$ is the polynomial defined in Lemma 5.25, and $\mathcal{U}$ is a complete set of representatives of isoclasses of modules $U$ over $\operatorname{supp} X \operatorname{such}$ that $\underline{\operatorname{dim}} U=\underline{\operatorname{dim}} X$ and $U \nsubseteq X$. Here we see that each $U \in \mathcal{U}$ cannot be homogeneous because if $U$ is homogeneous, the condition that $\underline{\operatorname{dim}} U=\underline{\operatorname{dim}} X$ implies $U \cong X$. Hence the right hand side is defined by Case 1 , and it is easy to see that this $\varphi_{X N}^{M}$ is a Hall polynomial.

Dually we have the following.

Proposition 5.28. Let $M$ and $N$ be preinjective A-modules. If $X$ is a non-sincere $A$-module, then there exists a Hall polynomial $\varphi_{N X}^{M}$.

This proposition proves the following. 
Corollary 5.29. Let $\left(x_{1}, x_{2}, \ldots, x_{n}\right)$ be a permutation of elements in $\Delta_{0}$ with $x_{1}=1$ such that $0 \neq\left[F_{x_{1}}, F_{x_{2}}, \ldots, F_{x_{n}}\right] \in \mathfrak{g}(\Delta)$. Set $f_{i}:=\delta-e_{x_{i}}$ for all $i=1, \ldots, n$. Then there exist Hall polynomials $\varphi_{M\left(f_{1}+\cdots+f_{i-1}\right), M\left(f_{i}\right)}^{M\left(f_{1}+\cdots+f_{i-1}+f_{i}\right)}$ for all $i=2, \ldots, n$.

\section{Proof of Main Theorem}

In this section we prove our main result Theorem 4.4. First we show the following.

6.a. Claim. There exists a homomorphism

$$
\phi: \mathfrak{g}(\Delta) \rightarrow L(A)_{1}^{\mathbb{C}} / I(A)^{\mathbb{C}}
$$

such that $\phi\left(E_{x}\right)=\varepsilon_{x}, \phi\left(F_{x}\right)=\zeta_{x}, \phi\left(H_{x}\right)=\eta_{x}$ for all $x \in \Delta_{0}$.

Indeed, it is enough to verify the following equations for all $x, y \in \Delta_{0}$ :

$$
\begin{aligned}
{\left[\eta_{x}, \eta_{y}\right] } & =0 \\
{\left[\varepsilon_{x}, \zeta_{x}\right] } & =\eta_{x} \\
{\left[\varepsilon_{x}, \zeta_{y}\right] } & =0, \quad \text { if } x \neq y \\
{\left[\eta_{x}, \varepsilon_{y}\right] } & =a_{x y} \varepsilon_{y} \\
{\left[\eta_{x}, \zeta_{y}\right] } & =-a_{x y} \zeta_{y} \\
\left(\operatorname{ad} \varepsilon_{x}\right)^{1-a_{x y}} \varepsilon_{y} & =0, \quad \text { if } x \neq y \\
\left(\operatorname{ad} \zeta_{x}\right)^{1-a_{x y}} \zeta_{y} & =0, \quad \text { if } x \neq y
\end{aligned}
$$

(see (3-1) for $\left.a_{x y}\right)$.

Verification of ( $(\underline{6-2})$. This follows from the construction of $\eta_{x}$ 's.

\section{Verification of (6-1).}

Lemma 6.1. Let $K \in \Omega$ and $\left(\mathcal{T}_{\rho}\right)_{\rho \in \mathbb{P}^{1}(K)}$ the tubular family in $\bmod A^{K}$. If $X$ and $Y$ are indecomposable $A^{K}$-modules contained in tubes $\mathcal{T}_{\rho}$ and $\mathcal{T}_{\sigma}$, respectively with $\rho \neq \sigma$, then $\left[u_{X}, u_{Y}\right]=0$ in $\bar{L}\left(A^{K}\right) /(|K|-1)$.

Proof. First note that there are no nonzero homomorphisms between indecomposable $A^{K}$-modules contained in distinct tubes because the tubular family $\left(\mathcal{T}_{\rho}\right)_{\rho \in \mathbb{P}^{1}(K)}$ is standard (Definition 3.5 (2)). If there exists an exact sequence of the form

$$
0 \rightarrow Y \stackrel{f}{\longrightarrow} M \stackrel{g}{\longrightarrow} X \rightarrow 0
$$

in $\bmod A$ with $M$ indecomposable, then $\operatorname{rank} M=\operatorname{rank} X+\operatorname{rank} Y=0$ shows that $M$ is contained in a tube $\mathcal{T}_{\lambda}\left(\lambda \in \mathbb{P}^{1}(K)\right)$. But $f \neq 0$ and $g \neq 0$ shows that $\rho=\lambda$ and $\lambda=\sigma$, thus $\rho=\sigma$, a contradiction. Hence there are no exact sequence of this form. By the symmetry of the argument the same statement still holds even if we exchange $X$ and $Y$. Hence $\left[u_{X}, u_{Y}\right]=0$ in $\bar{L}\left(A^{K}\right) /(|K|-1)$.

For each $K \in \Omega$ the lemma above shows that $\left[u_{W_{c}(K)}, u_{W_{c^{\prime}}(K)}\right]=0,\left[u_{W_{c}(K)}, u_{X_{i j}^{K}}\right]=0$, $\left[u_{X_{i j}^{K}}, u_{X_{s t}^{K}}\right]=0$ for all $c, c^{\prime} \in K \backslash E_{\Delta}, \alpha_{i j}, \alpha_{s t} \in Q_{1}$ with $c \neq c^{\prime}$ and $i \neq s$ (see (3-4)). It is trivial that $\left[u_{W_{c}(K)}, u_{W_{c}(K)}\right]=0$ for all $c \in K \backslash E_{\Delta}$, and also by Proposition 5.15 we have $\left[u_{X_{i j}^{K}}, u_{X_{i t}^{K}}\right]=0$ for all $\alpha_{i j}, \alpha_{i t} \in Q_{1}$. Therefore we have $\left[u_{X}, u_{Y}\right]=0$ for all 
indecomposable $A^{K}$-modules $X, Y$ with dimension vector $\delta$ and for all $K \in \Omega$. Hence by (4-2) and (4-4) we obtain the equation (6-1).

Verification of (6-3). Let $x, y \in \Delta_{0}$ with $x \neq y$. Assume that there exists an indecomposable $A$-module $M$ with $\underline{\operatorname{dim}} M=\underline{\operatorname{dim}} S_{x}+\underline{\operatorname{dim}} T_{y}=e_{x}+\delta-e_{y}$. Then $M$ is an indecomposable module over its support algebra $B:=\operatorname{supp} M=A / A \mathbf{e}_{y} A$ with dimension vector $w$, where $w_{z}=1$ if $z \neq x$; and $w_{z}=2$ if $z=x$ for all $z \in \Delta_{0} \backslash\{y\}$. If $y=1$, then $B$ is a hereditary algebra of Dynkin type $\Delta$. Since $w$ is a dimension vector of an indecomposable $B$-module, we must have $x=\infty$, a contradiction. Hence if $y=1$, then there exists no indecomposable $A$-modules of dimension vector $\underline{\operatorname{dim}} S_{x}+\underline{\operatorname{dim}} T_{y}$ , and we see that $\left[\varepsilon_{x}, \eta_{y}\right]=0$. Assume next that $y \neq 1$. Then since $w$ is a dimension vector of an indecomposable $B$-module, $\Delta$ is not of type $A_{n}$ and also $x=1$. In this case we see $\left[u_{S_{x}}, u_{T_{y}}\right]=(q-1) u_{M}$. Thus if we replace $k$ with any $K \in \Omega$, we have $\left[u_{S_{x}}, u_{T_{y}}\right]=(|K|-1) u_{M}=0$ in $\bar{L}\left(A^{K}\right) /(|K|-1)$. Hence in any case we have the equation (6-3).

Verification of ([6-6). Let $x, y \in \Delta_{0}$ with $x \neq y$.

Case 1. $x, y$ are not neighbors in $\Delta$. In this case $1-a_{x y}=1$, and we have to show $\left[\varepsilon_{x}, \varepsilon_{y}\right]=0$. Now since $x, y$ are not neighbors in $\Delta$, there exist no indecomposable $A$-modules of dimension vector $\underline{\operatorname{dim}} S_{x}+\underline{\operatorname{dim}} S_{y}$. This shows $\left[\varepsilon_{x}, \varepsilon_{y}\right]=0$.

Case 2. $x, y$ are neighbors in $\Delta$. In this case $1-a_{x y}=2$, and we have to show $\left[\varepsilon_{x},\left[\varepsilon_{x}, \varepsilon_{y}\right]\right]=0$. If this is nonzero, then there exists an indecomposable $A$-module $M$ with $\underline{\operatorname{dim}} M=2 \underline{\operatorname{dim}} S_{x}+\underline{\operatorname{dim}} S_{y}$. But the support algebra of $M$ is the algebra given by the full subquiver of $Q$ consisting of the vertices $x, y$, which is of type $A_{2}$. Over this algebra $M$ is still indecomposable but $\underline{\operatorname{dim}} M=(2,1)$, which is impossible.

Verification of (6-7). Let $x, y \in \Delta_{0}$ with $x \neq y$.

Case 1. $x, y$ are not neighbors in $\Delta$. By the formula (3-2) we see

$$
\chi\left(\underline{\operatorname{dim}} T_{x}+\underline{\operatorname{dim}} T_{y}\right)=\chi\left(2 \delta-e_{x}-e_{y}\right)=2 .
$$

Hence there exist no indecomposable $A$-modules of dimension vector $\underline{\operatorname{dim}} T_{x}+\underline{\operatorname{dim}} T_{y}$, which shows that $\left[\zeta_{x}, \zeta_{y}\right]=0$, as desired.

Case 2. $x, y$ are neighbors in $\Delta$. Again by the formula (3-2) we see

$$
\chi\left(2 \underline{\operatorname{dim}} T_{x}+\underline{\operatorname{dim}} T_{y}\right)=\chi\left(3 \delta-2 e_{x}-e_{y}\right)=7 .
$$

Hence similarly we have $\left[\zeta_{x},\left[\zeta_{x}, \zeta_{y}\right]\right]=0$, as desired.

Verification of (6-4). Let $x, y \in \Delta_{0}$. Since $\chi\left(\delta+e_{y}\right)=1$, we have an indecomposable $A$-module $M=M\left(\delta+e_{y}\right)$. By Proposition 5.22 we have $\overline{\mathbf{u}}_{M}=\varepsilon_{y}$. 
Case 1. $y \neq 1$, say $y=x_{\text {st }}$ for some $s, t$ with $t>1$. In this case we may assume that $M=(M(z), M(\alpha))_{z \in Q_{0}, \alpha \in Q_{1}}$ has the following structure:

$$
\begin{gathered}
M(z)= \begin{cases}k^{2} & \text { if } z=x_{s t} \\
k & \text { otherwise, }\end{cases} \\
M\left(\alpha_{i j}\right)= \begin{cases}(0, \mathbb{1}) & \text { if }(i, j)=(s, t-1) ; \\
\left(\begin{array}{ll}
1 \\
0
\end{array}\right) & \text { if }(i, j)=(s, t) ; \\
-\mathbb{1} & \text { if }(i, s, t) \in\{(1,3,1),(2,3,1),(3,2,1)\} ; \\
\mathbb{1} & \text { otherwise }\end{cases}
\end{gathered}
$$

because this gives an indecomposable $A$-module of dimension vector $\delta+e_{y}$. Now since soc $M \cong S_{1} \oplus S_{y}$, and top $M \cong S_{y} \oplus S_{\infty}$, we have

$$
\mathcal{F}_{S_{y}, *}^{M}=\{N\}=\mathcal{F}_{S_{y}, X_{s, t-1}}^{M}, \text { and } \mathcal{F}_{*, S_{y}}^{M}=\{S\}=\mathcal{F}_{X_{s t}, S_{y}}^{M}
$$

for some $N \cong X_{s, t-1}$ and $S \cong S_{y}$. Therefore we have the following formula: For any $K \in \Omega$,

$$
\begin{aligned}
{\left[u_{X_{i j}^{K}}, u_{S_{x_{s t}}^{K}}\right] } & = \begin{cases}u_{M^{K}} & \text { if }(i, j)=(s, t) ; \\
-u_{M^{K}} & \text { if }(i, j)=(s, t-1) ; \\
0 & \text { otherwise; and }\end{cases} \\
{\left[u_{W_{c}(K)}, u_{S_{x_{s t}}^{K}}\right] } & =0 \text { for all } c \in K \backslash E_{\Delta}
\end{aligned}
$$

in $\bar{L}\left(A^{K}\right) /(|K|-1)$, where $E_{\Delta}$ is as in $(3-4)$.

Case 1.1. $x \neq 1$, say $x=x_{i j}$ for some $i, j$. In this case $\eta_{x}$ has the form (4-4).

Case 1.1.1. $x=y$. In this case $a_{x y}=2$. By the formula (6-8) we have

$$
\begin{aligned}
{\left[u_{X_{i j}}, u_{S_{x_{i j}}}\right] } & =u_{M} \\
{\left[u_{X_{i, j-1}}, u_{S_{X_{i j}}}\right] } & =-u_{M}
\end{aligned}
$$

Therefore $\left[u_{X_{i j}}-u_{X_{i, j-1}}, u_{S_{x_{i j}}}\right]=2 u_{M}$. This shows that $\left[\eta_{x}, \varepsilon_{y}\right]=2 \varepsilon_{y}=a_{x y} \varepsilon_{y}$.

Case 1.1.2. $x \neq y$ and $x, y$ are not neighbors in $\Delta$. In this case $a_{x y}=0$. Again by (6-8) we have $\left[u_{X_{i j}}, u_{X_{y}}\right]=0=\left[u_{X_{i, j-1}}, u_{S_{y}}\right]$. Thus $\left[\eta_{x}, \varepsilon_{y}\right]=0=a_{x y} \varepsilon_{y}$.

Case 1.1.3. $x \neq y$ and $x, y$ are neighbors in $\Delta$. In this case $a_{x y}=-1$, and $x \in$ $\left\{x_{s, t-1}, x_{s, t+1}\right\}$. When $x=x_{s, t-1}$, it follows from $\left[u_{X_{s, t-1}}, u_{S_{x_{s t}}}\right]=-u_{M}$ and $\left[u_{X_{s, t-2}}, u_{S_{x_{s t}}}\right]=$ 0 (by (6-8) ) that $\left[\eta_{x}, \varepsilon_{y}\right]=-\varepsilon_{y}=a_{x y} \varepsilon_{y}$. When $x=x_{s, t+1}$, it follows from $\left[u_{X_{s, t+1}}, u_{S_{x_{s t}}}\right]=$ 0 and $\left[u_{X_{s t}}, u_{S_{x_{s t}}}\right]=u_{M}$ that $\left[\eta_{x}, \varepsilon_{y}\right]=-\varepsilon_{y}=a_{x y} \varepsilon_{y}$.

Case 1.2. $x=1$. In this case $x \neq y$ and $\eta_{x}$ has the form (4-2).

Case 1.2.1. $x, y$ are not neighbors in $\Delta$. Then $a_{x y}=0$ and $t \geq 3$. By (6-8) we have $\left[\eta_{x}, \varepsilon_{y}\right]=0=a_{x y} \varepsilon_{y}$.

Case 1.2.2. $x, y$ are neighbors in $\Delta$. Then $a_{x y}=-1$ and $t=2$. By (6-8) we have $\left[\eta_{x}, \varepsilon_{y}\right]=-\varepsilon_{y}=a_{x y} \varepsilon_{y}$. 
Case 2. $y=1$. In this case we may assume that $M$ has the following structure:

$$
\begin{aligned}
& M(z)= \begin{cases}k^{2} & \text { if } z=1 \\
k & \text { otherwise }\end{cases}
\end{aligned}
$$

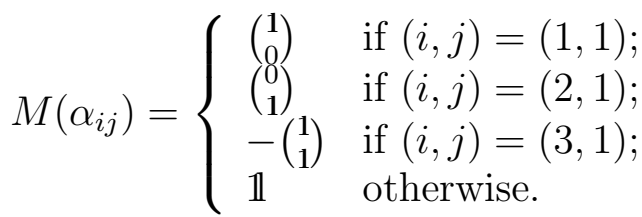

Then as easily seen for any $K \in \Omega$ we have

$$
\begin{aligned}
& F_{X, S_{1}^{K}}^{M^{K}}=\left\{\begin{array}{l}
1 \text { if } X \cong W_{c}(K) \text { (for some } c \in K \text { ) or } X_{11}^{K} ; \\
0 \text { otherwise. }
\end{array}\right. \\
& F_{S_{1}^{K}, X}^{M^{K}}=0 \text { for all incecomposable } X \text { with } \underline{\operatorname{dim}} X=\delta .
\end{aligned}
$$

Case 2.1. $x \neq 1$, say $x=x_{i j}$. Then $x \neq y$ and $\eta_{x}$ has the form (4-4).

Case 2.1.1. $x, y$ are not neighbors in $\Delta$. In this case $a_{x y}=0$ and $j \geq 3$. By (6-9) we have $\left[\eta_{x}, \varepsilon_{y}\right]=0=a_{x y} \varepsilon_{y}$.

Case 2.1.2. $x, y$ are neighbors in $\Delta$. In this case $a_{x y}=-1$ and $x=x_{i 2}$. By (6-9) we have $\left[u_{X_{i 2}}, u_{S_{1}}\right]=0$ and $\left[u_{X_{i 1}}, u_{S_{1}}\right]=u_{M}$ and hence $\left[\eta_{x}, \varepsilon_{y}\right]=-\varepsilon_{y}=a_{x y} \varepsilon_{y}$.

Case 2.2. $x=1$. Then $x=y=1$ and $a_{x y}=2$. By ([6-9) we have for any $K \in \Omega$

$$
\left[\sum_{c \in K} u_{W_{c}(K)}+u_{X_{11}^{K}}, u_{S_{1}^{K}}\right]=(|K|+1) u_{M^{K}}=2 u_{M^{K}}
$$

in $\bar{L}\left(A^{K}\right) /(|K|-1)$. Therefore $\left[\eta_{1}, \varepsilon_{1}\right]=2 \varepsilon_{1}=a_{11} \varepsilon_{1}$.

Verification of (6-5) . Set $f_{x}:=\delta-e_{x}$ for all $x \in Q_{0}$. First we show the following.

Lemma 6.2. For each $x \in \Delta_{0}$ we have $\overline{\mathbf{u}}_{m\left(f_{x}+\delta\right)}=\overline{\mathbf{u}}_{m\left(f_{x}\right)}$.

Proof. If $x \neq 1$, then $f_{x}+\delta$ is a positive root of $\chi_{A}$ with $M\left(f_{x}+\delta\right)$ a regular module, and $\operatorname{org}\left(f_{x}+\delta\right)=f_{x}$. Hence the assertion holds by Proposition 5.18. Now let $x=1$. First we assume that $\Delta$ is not of type $A_{n}$. We may assume that the module $M:=M\left(f_{1}+\delta\right)$ has the following structure:

$$
\begin{gathered}
M(z)= \begin{cases}k & \text { if } z=1 ; \\
k^{2} & \text { otherwise. }\end{cases} \\
M\left(\alpha_{i j}\right)= \begin{cases}(1,0) & \text { if }(i, j)=(1,1) ; \\
(0,1) & \text { if }(i, j)=(2,1) ; \\
-(1,1) & \text { if }(i, j)=(3,1) ; \\
\mathbb{1}_{k^{2}} & \text { otherwise. }\end{cases}
\end{gathered}
$$


By looking at this structure we easily see that the following equalities hold:

$$
\begin{aligned}
& \mathbf{u}_{m\left(f_{1}+\delta\right)}=\left[\mathbf{u}_{m\left(e_{\infty}+\delta\right)}, \mathbf{u}_{m\left(e_{x_{32}}\right)}, \mathbf{u}_{m\left(e_{x_{2, p(2)}}\right)}, \mathbf{u}_{m\left(e_{x_{2, p(2)-1}}\right)}, \ldots, \mathbf{u}_{m\left(e_{x_{22}}\right)},\right. \\
& \left.\mathbf{u}_{m\left(e_{x_{1, p(1)}}\right)}, \mathbf{u}_{m\left(e_{x_{1, p(1)-1}}\right)}, \ldots, \mathbf{u}_{m\left(e_{x_{12}}\right)}\right] ; \text { and } \\
& \mathbf{u}_{m\left(f_{1}\right)}=\left[\mathbf{u}_{m\left(e_{\infty}\right)}, \mathbf{u}_{m\left(e_{x_{32}}\right)}, \mathbf{u}_{m\left(e_{x_{2, p(2)}}\right)}, \mathbf{u}_{m\left(e_{x_{2, p(2)-1}}\right)}, \ldots, \mathbf{u}_{m\left(e_{x_{22}}\right)},\right.
\end{aligned}
$$

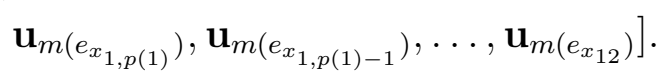

Hence we have

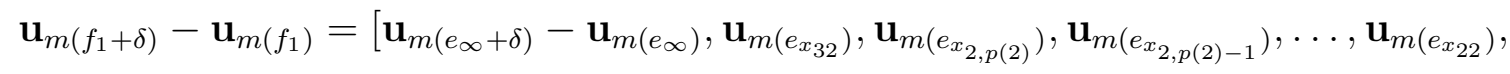

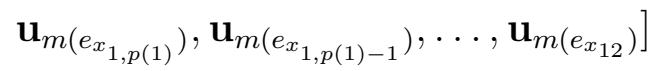

is in $I(A)^{\mathbb{C}}$ because so is $\mathbf{u}_{m\left(e_{\infty}+\delta\right)}-\mathbf{u}_{m\left(e_{\infty}\right)}$ by Proposition 5.22 .

Finally a similar argument works also in the $A_{n}$ case.

Case 1. $y \neq 1$, say $y=x_{i j}(i \in\{1, \ldots, r\}, j \in\{2, \ldots, p(i)\})$. In this case $\zeta_{y}=\overline{\mathbf{u}}_{m\left(f_{y}\right)}$ and $M\left(f_{y}\right)$ is a regular exceptional module contained in a non-homogeneous tube $\mathcal{T}_{\rho}$. Note that for each $s \in\{1, \ldots, r\}$ and $t \in\{2, \ldots, p(s)\}$ we have $X_{s t} \in \mathcal{T}_{\rho}$ if and only if $s=i$. By Lemma 6.1 we have

$$
\begin{cases}{\left[u_{W_{c}(K)}, u_{m\left(f_{y}\right)^{K}}\right]=0} & \forall c \in K \backslash E_{\Delta} ; \text { and } \\ {\left[u_{X_{x t}^{K}}, u_{m\left(f_{y}\right)^{K}}\right]=0} & \forall s \in\{1, \ldots, r\} \backslash\{i\}, \forall t \in\{2, \ldots, p(s)\} .\end{cases}
$$

If $s=i$, then we can calculate the bracket $\left[u_{X_{i t}^{K}}, u_{m\left(f_{y}\right)^{K}}\right]$ in $\mathcal{T}_{\rho}$ using (5-3) as follows.

$$
\left[u_{X_{i t}^{K}}, u_{m\left(f_{y}\right)^{K}}\right]= \begin{cases}-u_{m\left(f_{y}+\delta\right)^{K}} & \text { if } j=t ; \\ u_{m\left(f_{y}+\delta\right)^{K}} & \text { if } j=t+1 ; \text { and } \\ 0 & \text { otherwise. }\end{cases}
$$

Using this we now calculate $\left[\eta_{x}, \zeta_{y}\right]$.

Case 1.1. $x=1$. For any $K \in \Omega$, we have

$$
\begin{aligned}
{\left[\sum_{c \in K \backslash E_{\Delta}} u_{W_{c}(K)}+u_{X_{11}^{K}}+\cdots+u_{X_{r 1}^{K}}, u_{m\left(f_{y}\right)^{K}}\right] } & =\left[u_{X_{i 1}^{K}}, u_{m\left(f_{y}\right)^{K}}\right] \\
& = \begin{cases}-u_{m\left(f_{y}+\delta\right)^{K}} & \text { if } j=1 \text { (i.e., } y=1, \text { impossible) } \\
u_{m\left(f_{y}+\delta\right)^{K}} & \text { if } \left.j=2 \text { (i.e., } y=x_{i 2}\right) \\
0 & \text { otherwise (i.e., } \left.y=x_{i j}, j \geq 3\right) \\
& =-a_{x y} u_{m\left(f_{y}+\delta\right)^{K}} .\end{cases}
\end{aligned}
$$

Hence we have $\left[\eta_{x}, \zeta_{y}\right]=-a_{x y} \zeta_{y}$ by Lemma 6.2. 
Case 1.2. $x \neq 1$, say $x=x_{s t}(s \in\{1, \ldots, r\}$ and $t \in\{2, \ldots, p(s)\})$. Let $K \in \Omega$. If $s \neq i$, then $a_{x y}=0$ and $\left[u_{X_{s t}^{K}}-u_{X_{s, t-1}^{K}}, u_{m\left(f_{y}\right)^{K}}\right]=0=-a_{x y} u_{m\left(f_{y}\right)^{K}}$. Thus in this case we have $\left[\eta_{x}, \zeta_{y}\right]=-a_{x y} \zeta_{y}$. Therefore we may assume that $s=i$.

$$
\begin{aligned}
{\left[u_{X_{s t}^{K}}-u_{X_{s, t-1}^{K}}, u_{m\left(f_{y}\right)^{K}}\right] } & = \begin{cases}-u_{m\left(f_{y}+\delta\right)^{K}} & \text { if } j=t \\
u_{m\left(f_{y}+\delta\right)^{K}} & \text { if } j=t+1 \\
0 & \text { otherwise }\end{cases} \\
& + \begin{cases}-u_{m\left(f_{y}+\delta\right)^{K}} & \text { if } j=t-1 \\
u_{m\left(f_{y}+\delta\right)^{K}} & \text { if } j=t \\
0 & \text { otherwise }\end{cases} \\
= & -a_{x y} u_{m\left(f_{y}+\delta\right)^{K}} .
\end{aligned}
$$

Hence also in this case we have $\left[\eta_{x}, \zeta_{y}\right]=-a_{x y} \zeta_{y}$ by Lemma 6.2.

Case 2. $y=1$. In this case $\zeta_{1}=-\overline{\mathbf{u}}_{m\left(f_{1}\right)}$. Using the structure of $M\left(f_{1}+\delta\right)$ described in (6-10) we can calculate Hall numbers as follows: For any $K \in \Omega$

$$
\begin{gathered}
F_{M\left(f_{1}\right)^{K} X_{i j}^{K}}^{M\left(f_{1}+\delta\right)^{K}}= \begin{cases}1 & \text { if } j=1 ; \\
0 & \text { otherwise. }\end{cases} \\
F_{M\left(f_{1}\right)^{K} W_{c}(K)}^{M\left(f_{1}+\right)^{K}}=1 \quad \text { for all } c \in K .
\end{gathered}
$$

Further since there are no nonzero homomorphisms from preinjectives to regulars, we have

$$
F_{X, M\left(f_{1}\right)^{K}}^{M\left(f_{1}+\delta\right)^{K}}=0 \quad \text { for all indecomposable } X \text { with } \underline{\operatorname{dim}} X=\delta .
$$

These enable us to calculate $\left[\eta_{x}, \zeta_{1}\right]$.

Case 2.1. $x=1$. In this case $a_{11}=2$. For any $K \in \Omega$ we have

$$
\left[\sum_{c \in K} u_{W_{c}(K)}+u_{X_{11}^{K}}, u_{m\left(f_{1}\right)^{K}}\right]=-(|K|+1) u_{m\left(f_{1}+\delta\right)^{K}}=-2 u_{m\left(f_{1}+\delta\right)^{K}}
$$

in $\bar{L}\left(A^{K}\right) /(|K|-1)$. Hence by Lemma 6.2 we have $\left[\eta_{1}, \zeta_{1}\right]=-a_{11} \zeta_{1}$.

Case 2.2. $x, y$ are neighbors in $\Delta$, i.e., $x=x_{i j}$ with $j=2$. In this case $a_{x 1}=-1$. For any $K \in \Omega$ we have

$$
\left[u_{X_{i 2}^{K}}-u_{X_{i 1}^{K}}, u_{m\left(f_{1}\right)^{K}}\right]=u_{m\left(f_{1}+\delta\right)^{K}} .
$$

Hence by Lemma 6.2 we have $\left[\eta_{x}, \zeta_{1}\right]=-a_{x 1} \zeta_{1}$.

Case 2.3. $x, y$ are not neighbors in $\Delta$, i.e., $x=x_{i j}$ with $j \geq 3$. In this case $a_{x 1}=0$. For any $K \in \Omega$ we have

$$
\left[u_{X_{i j}^{K}}-u_{X_{i, j-1}^{K}}, u_{m\left(f_{1}\right)^{K}}\right]=0 .
$$

Hence by Lemma 6.2 we have $\left[\eta_{x}, \zeta_{1}\right]=-a_{x 1} \zeta_{1}$. This finishes the proof of the claim. 
6.b. Injectivity of $\phi$. We next show that $\phi: \mathfrak{g}(\Delta) \rightarrow L(A)_{1}^{\mathbb{C}} / I(A)^{\mathbb{C}}$ is injective. Since $\mathfrak{g}(\Delta)$ is simple, it is enough to show that $\operatorname{Im} \phi \neq 0$. First we consider the case that $\Delta \neq A_{1}$. In this case $x_{12}$ exists, and we set $e_{2}:=e_{x_{12}}, E_{2}:=E_{x_{12}}$ for short. Then it is enough to show that $\phi\left(E_{2}\right) \neq 0$, i.e., that $\mathbf{u}_{m\left(e_{2}\right)} \otimes 1_{\mathbb{C}} \notin I(A)^{\mathbb{C}}$, where $1_{\mathbb{C}}$ stands for the identity $1 \in \mathbb{C}$. Denote by $1_{\mathbb{Q}}$ the identity $1 \in \mathbb{Q}$. Since the canonical isomorphism $\left(L(A)_{1}^{\mathbb{Q}} / I(A)^{\mathbb{Q}}\right)^{\mathbb{C}} \stackrel{\sim}{\rightarrow} L(A)_{1}^{\mathbb{C}} / I(A)^{\mathbb{C}}$ sends the coset of $\mathbf{u}_{m\left(e_{2}\right)} \otimes 1_{\mathbb{Q}}$ to that of $\mathbf{u}_{m\left(e_{2}\right)} \otimes 1_{\mathbb{C}}$, we have only to show that $\mathbf{u}_{m\left(e_{2}\right)} \otimes 1_{\mathbb{Q}} \notin I(A)^{\mathbb{Q}}$. Assume that $\mathbf{u}_{m\left(e_{2}\right)}=\mathbf{u}_{m\left(e_{2}\right)} \otimes 1_{\mathbb{Q}} \in I(A)^{\mathbb{Q}}$. Then by definition of $I(A)$ there exist a finite set $J \subseteq \bigcup_{n \in \mathbb{N}} Q_{0}^{(n)}$ and an $\left(a_{i}\right)_{i \in J} \in \mathbb{Q}^{J}$ such that $\mathbf{u}_{m\left(e_{2}\right)}$ is expressed as a linear combination

$$
\mathbf{u}_{m\left(e_{2}\right)}=\sum_{i \in J} a_{i}\left[\mathbf{u}_{m\left(e_{i(1)}\right)}, \mathbf{u}_{m\left(e_{i(2)}\right)}, \ldots, \mathbf{u}_{m\left(e_{i\left(t_{i}-1\right)}\right)}, \mathbf{u}_{m\left(e_{i\left(t_{i}\right)}+\delta\right)}-\mathbf{u}_{m\left(e_{i\left(t_{i}\right)}\right)}\right]
$$

where we put $i=\left(i(1), \ldots, i\left(t_{i}\right)\right)$ for all $i \in J$. Take an $a \in \mathbb{N}$ such that $a a_{i} \in \mathbb{Z}$ for all $i \in J$. By renaming $a a_{i}$ as $a_{i}$, we have

$$
a \mathbf{u}_{m\left(e_{2}\right)}=\sum_{i \in J} a_{i}\left[\mathbf{u}_{m\left(e_{i(1)}\right)}, \mathbf{u}_{m\left(e_{i(2)}\right)}, \ldots, \mathbf{u}_{m\left(e_{i\left(t_{i}-1\right)}\right)}, \mathbf{u}_{m\left(e_{i\left(t_{i}\right)}+\delta\right)}-\mathbf{u}_{m\left(e_{i\left(t_{i}\right)}\right)}\right],
$$

with $a_{i} \in \mathbb{Z}$ for all $i \in J$. We put

$$
d_{i}:=\sum_{j=1}^{t_{i}} e_{i(j)} \quad \text { and } \quad e(i):=e_{i\left(t_{i}\right)}
$$

for all $i \in J$. Then for each $K \in \Omega$ we have

$$
\begin{aligned}
& a u_{m\left(e_{2}\right)^{K}}=\sum_{i \in J} a_{i}\left[u_{m\left(e_{i(1)}\right)^{K}}, u_{m\left(e_{i(2)}\right)^{K}}, \ldots, u_{m\left(e_{i\left(t_{i}-1\right)}\right)^{K}}, u_{m(e(i)+\delta)^{K}}-u_{m(e(i))^{K}}\right] \\
& =\sum_{i \in J_{1}} a_{i}\left[u_{m\left(e_{i(1)}\right)^{K}}, u_{m\left(e_{i(2)}\right)^{K}}, \ldots, u_{m\left(e_{i\left(t_{i}-1\right)}\right)^{K}}, u_{m(e(i)+\delta)^{K}}-u_{m(e(i))^{K}}\right] \\
& +\sum_{i \in J_{2}} a_{i}\left[u_{m\left(e_{i(1)}\right)^{K}}, u_{m\left(e_{i(2)}\right)^{K}}, \ldots, u_{m\left(e_{i\left(t_{i}-1\right)}\right)^{K}}, u_{m(e(i)+\delta)^{K}}-u_{m(e(i))^{K}}\right],
\end{aligned}
$$

in $\bar{L}\left(A^{K}\right) /(|K|-1)$ where $J_{1}:=\left\{i \in J \mid d_{i} \in \mathbb{Z} \delta+e_{2}\right\}$ and $J_{2}:=\left\{i \in J \mid d_{i} \notin \mathbb{Z} \delta+e_{2}\right\}$. Here since $\bar{L}\left(A^{K}\right) /(|K|-1)$ is a free $\mathbb{Z} /(|K|-1)$-module with basis [ind $A^{K}$ ] which is a disjoint union of the subsets $\left\{[X] \in\left[\operatorname{ind} A^{K}\right] \mid \underline{\operatorname{dim}} X \in \mathbb{Z} \delta+e_{2}\right\}$ and $\left\{[X] \in\left[\operatorname{ind} A^{K}\right] \mid\right.$ $\left.\underline{\operatorname{dim}} X \notin \mathbb{Z} \delta+e_{2}\right\}$, we have

$$
a u_{m\left(e_{2}\right)^{K}}=\sum_{i \in J_{1}} a_{i}\left[u_{m\left(e_{i(1)}\right)^{K}}, u_{m\left(e_{i(2)}\right)^{K}}, \ldots, u_{m\left(e_{i\left(t_{i}-1\right)}\right)^{K}}, u_{m(e(i)+\delta)^{K}}-u_{m(e(i))^{K}}\right] .
$$

We can write $d_{i}=s_{i} \delta+e_{2}$ for some $s_{i} \in \mathbb{Z}$. Now we have

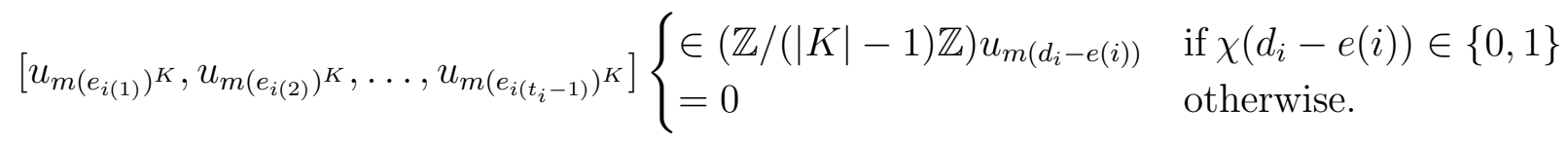


Here,

$$
\begin{aligned}
\chi\left(d_{i}-e(i)\right) & =B\left(s_{i} \delta+e_{2}-e(i), s_{i} \delta+e_{2}-e(i)\right) \\
& =s_{i}^{2} B(\delta, \delta)+s_{i}\left\{B\left(\delta, e_{2}-e(i)\right)+B\left(e_{2}-e(i), \delta\right)\right\}+B\left(e_{2}-e(i), e_{2}-e(i)\right) \\
& =B\left(e_{2}, e_{2}\right)-\left\{B\left(e_{2}, e(i)\right)+B\left(e(i), e_{2}\right)\right\}+B(e(i), e(e)) \\
& \in 2-\{2,0,-1\}=\{0,2,3\} .
\end{aligned}
$$

Hence $\chi\left(d_{i}-e(i)\right) \in\{0,1\} \Leftrightarrow \chi\left(d_{i}-e(i)\right)=0 \Leftrightarrow B\left(e_{2}, e(i)\right)+B\left(e(i), e_{2}\right)=2 \Leftrightarrow e(i)=e_{2}$. Therefore by putting $J_{0}:=\left\{i \in J_{1} \mid e(i)=e_{2}\right\}=\left\{i \in J \mid d_{i} \in \mathbb{Z} \delta+e_{2}, e(i)=e_{2}\right\}$ we have

$$
a u_{m\left(e_{2}\right)^{K}}=\sum_{i \in J_{0}} a_{i}\left[u_{m\left(e_{i(1)}\right)^{K}}, u_{m\left(e_{i(2)}\right)^{K}}, \ldots, u_{m\left(e_{i\left(t_{i}-1\right)}\right)^{K}}, u_{m\left(e_{2}+\delta\right)^{K}}-u_{m\left(e_{2}\right)^{K}}\right] .
$$

For each $i \in J_{0}$ set $I_{i}:=\left\{[X] \in\left[\operatorname{ind} A^{K}\right] \mid \underline{\operatorname{dim}} X=s_{i} \delta\right\}$. Then noting that $d_{i}-e_{2}=s_{i} \delta$ for all $i \in J_{0}$, we see for each $i \in J_{0}$ and for each $[X] \in I_{i}$ there exists some $b_{i,[X]} \in$ $\mathbb{Z} /(|K|-1) \mathbb{Z}$ such that

$$
\left[u_{m\left(e_{i(1)}\right)^{K}}, u_{m\left(e_{i(2)}\right)^{K}}, \ldots, u_{\left.m\left(e_{i\left(t_{i}-1\right)}\right)^{K}\right]}=\sum_{[X] \in I_{i}} b_{i,[X]} u_{[X]} .\right.
$$

Then

$$
\begin{aligned}
& {\left[u_{m\left(e_{i(1)}\right)^{K}}, u_{m\left(e_{i(2)}\right)^{K}}, \ldots, u_{m\left(e_{i\left(t_{i}-1\right)}\right)^{K}}, u_{m\left(e_{2}+\delta\right)^{K}}\right]=\sum_{[X] \in I_{i}} b_{i,[X]}\left[u_{[X]}, u_{m\left(e_{2}+\delta\right)^{K}}\right]} \\
& =\sum_{[X] \in I_{i}} b_{i,[X]} b_{[X], m\left(e_{2}+\delta\right)^{K}} u_{m\left(e_{2}+\left(s_{i}+1\right) \delta\right)^{K}},
\end{aligned}
$$

and

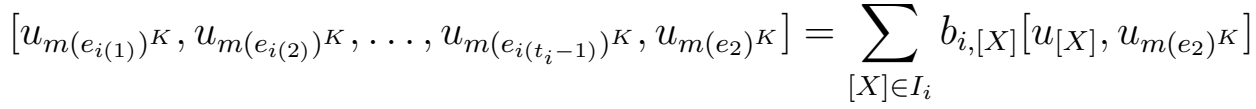

$$
\begin{aligned}
& =\sum_{[X] \in I_{i}} b_{i,[X]} b_{[X], m\left(e_{2}\right)^{K}} u_{m\left(e_{2}+s_{i} \delta\right)^{K}} .
\end{aligned}
$$

Here by Proposition [5.23, we have $b_{[X], m\left(e_{2}+\delta\right)^{K}}=b_{[X], m\left(e_{2}\right)^{K}}$ for all $[X] \in I_{i}$. Hence we obtain

$\left[u_{m\left(e_{i(1)}\right)^{K}}, u_{m\left(e_{i(2)}\right)^{K}}, \ldots, u_{m\left(e_{i\left(t_{i}-1\right)}\right)^{K}}, u_{m\left(e_{2}+\delta\right)^{K}}-u_{m\left(e_{2}\right)^{K}}\right]=c_{i}(K)\left(u_{m\left(e_{2}+\left(s_{i}+1\right) \delta\right)^{K}}-u_{\left.m\left(e_{2}+s_{i} \delta\right)^{K}\right)}\right)$

where we put $c_{i}(K):=\sum_{[X] \in I_{i}} b_{i,[X]} b_{[X], m\left(e_{2}\right)^{K}} \in \mathbb{Z} /(|K|-1) \mathbb{Z}$. As a consequence, we have

$$
a u_{m\left(e_{2}\right)^{K}}=\sum_{i \in J_{0}} a_{i} c_{i}(K)\left(u_{m\left(e_{2}+\left(s_{i}+1\right) \delta\right)^{K}}-u_{m\left(e_{2}+s_{i} \delta\right)^{K}}\right) .
$$

From this formula, it is easy to see that $a=0$ in $\mathbb{Z} /(|K|-1) \mathbb{Z}$ for all $K \in \Omega$. Hence $a=0$ in $\mathbb{Z}$, a contradiction. Hence we must have $\mathbf{u}_{m\left(e_{2}\right)} \notin I(A)^{\mathbb{Q}}$, and hence $\operatorname{Im} \phi \neq 0$.

Also in the case that $\Delta=A_{1}$, a similar argument works by Proposition 5.24 to show that $\operatorname{Im} \phi \neq 0$. 
6.c. Surjectivity of $\phi$. We finally show that $\phi$ is surjective. It is enough to show that $\varepsilon_{\infty} \in \operatorname{Im} \phi$ because $L(A)_{1}^{\mathbb{C}} / I(A)^{\mathbb{C}}$ is generated by $\left\{\varepsilon_{x} \mid x \in Q_{0}\right\}$ and we already know that $\left\{\varepsilon_{x} \mid x \in Q_{0} \backslash\{\infty\}\right\} \subseteq \operatorname{Im} \phi$ by definition of $\phi$. There exists a permutation $\left(x_{1}, \ldots, x_{n}\right)$ of $\Delta_{0}$ such that $\left[F_{x_{1}}, \cdots, F_{x_{n}}\right] \neq 0$ in $\mathfrak{g}(\Delta)$ (see 1.a). Thus by 6.b we have $\left[\zeta_{x_{1}}, \ldots, \zeta_{x_{n}}\right] \neq 0$ in $L(A)_{1}^{\mathbb{C}} / I(A)^{\mathbb{C}}$. Set $f_{x}:=\delta-e_{x}=\underline{\operatorname{dim}} T_{x}$ for all $x \in \Delta_{0}$ and note that

$$
\sum_{x \in \Delta_{0}} f_{x}=\sum_{x \in \Delta_{0}}\left(\delta-e_{x}\right)=(n-1) \delta+e_{\infty}
$$

Since $B\left(\delta, e_{\infty}\right)=1, B\left(e_{\infty}, \delta\right)=-1$ and $B\left(e_{\infty}, e_{\infty}\right)=1$ we have $\chi\left((n-1) \delta+e_{\infty}\right)=1$. Hence there exists a unique indecomposable $A$-module $M$ with $\underline{\operatorname{dim}} M=(n-1) \delta+e_{\infty}$ up to isomorphisms. Hence we have

$$
\left[\zeta_{x_{1}}, \ldots, \zeta_{x_{n}}\right]=\overline{\left(c(K) u_{\left[M^{K}\right]}\right)_{K \in \Omega}}
$$

for some $c(K) \in \mathbb{Z} /(|K|-1) \mathbb{Z}$ for each $K \in \Omega$. By Corollary [5.29, there exist Hall polynomials

$$
\varphi_{M\left(f_{x_{1}}\right) M\left(f_{x_{2}}\right)}^{M\left(f_{x_{1}}+f_{x_{2}}\right)}, \varphi_{M\left(f_{x_{3}}\right) M\left(f_{x_{1}}+f_{x_{2}}\right)}^{M\left(f_{x_{1}}+f_{x_{2}}+f_{x_{3}}\right)}, \ldots, \varphi_{M\left(f_{x_{n}}\right) M\left(\sum_{i=1}^{n-1} f_{x_{i}}\right)}^{M} .
$$

Therefore there is some $c \in \mathbb{Z}$ such that $c \equiv c(K)(\bmod |K|-1)$ for all $K \in \Omega$. Thus $0 \neq\left[\zeta_{x_{1}}, \ldots, \zeta_{x_{n}}\right]=c \overline{\mathbf{u}}_{M}$ and we have $c \neq 0$. By Proposition 5.22, we have $\overline{\mathbf{u}}_{M}=\overline{\mathbf{u}}_{m\left(e_{\infty}\right)}=\varepsilon_{\infty}$. Hence $\varepsilon_{\infty}=\frac{1}{c}\left[\zeta_{x_{1}}, \ldots, \zeta_{x_{n}}\right] \in \operatorname{Im} \phi$.

As a consequence, $\phi: \mathfrak{g}(\Delta) \rightarrow L(A)_{1}^{\mathbb{C}} / I(A)^{\mathbb{C}}$ is an isomorphism.

\section{Root spaces}

In this section we prove Proposition 4.8 .

7.a. Gabriel-Roiter submodules. We first recall the definitions of the GabrielRoiter measure and of Gabriel-Roiter submodules (see Ringel [28] for details).

Definition 7.1. Let $M \in \bmod A$ and $l$ the length of $M$.

(1) The Gabriel-Roiter measure $\mu(M) \in \mathbb{Q}$ of $M$ is defined by induction on $l$ as follows. If $l=0$, then $\mu(M):=0$. If $l>0$, then

$$
\mu(M):=\max _{M^{\prime}<M} \mu\left(M^{\prime}\right)+ \begin{cases}2^{-l} & \text { if } M \text { is indecomposable; } \\ 0 & \text { otherwise, }\end{cases}
$$

where $M^{\prime}$ runs through all proper submodules of $M$ (for the existence of this maximum see [28, Section 1]).

(2) If $M$ is indecomposable and $M^{\prime}$ is an indecomposable submodule of $M$ with $\mu\left(M^{\prime}\right)$ maximal, then we call $M^{\prime}$ a Gabriel-Roiter submodule (GR-submodule for short) of $M$ and the embedding $M^{\prime} \hookrightarrow M$ a Gabriel-Roiter inclusion (GR-inclusion for short).

(3) A monomorphism $u$ is called mono-irreducible if (i) $u$ is not a section, and (ii) for every factorization $u=u^{\prime \prime} u^{\prime}$ with $u^{\prime \prime}$ a monomorphism, either $u^{\prime}$ is a section or $u^{\prime \prime}$ is an isomorphism.

We cite the following from [28, Section 2]. 
Proposition 7.2. (1) GR-inclusions are mono-irreducible.

(2) The cokernel of a mono-irreducible monomorphism between indecomposable Amodules is indecomposable.

(3) Let $Y$ be an indecomposable A-module with a GR-submodule $X$ and let $U$ be a submodule of $Y$ isomorphic to $X$. Then $U$ is also a GR-submodule of $Y$, and hence $Y / U$ is again indecomposable by (1) and (2) above.

Lemma 7.3. Let $Y$ be an indecomposable A-module with a GR-submodule $X$. If $\underline{\operatorname{dim}} Y / X \notin \mathbb{Z} \delta$, then $\mathcal{F}_{*, X}^{Y}=\mathcal{F}_{Y / X, X}^{Y}$.

Proof. It is enough to show that $\mathcal{F}_{*, X}^{Y} \subseteq \mathcal{F}_{Y / X, X}^{Y}$. Let $U \in \mathcal{F}_{*, X}^{Y}$. Then since $Y \geq$ $U \cong X$, both $Y / X$ and $Y / U$ are indecomposable by the proposition above. But since $\underline{\operatorname{dim}} Y / U=\underline{\operatorname{dim}} Y / X \notin \mathbb{Z} \delta$, we have $Y / U \cong Y / X$. Hence $U \in \mathcal{F}_{Y / X, X}^{Y}$.

Proposition 7.4. Let $Y$ be a preprojective indecomposable A-module with a GRsubmodule $X$. If $\underline{\operatorname{dim}} Y / X \notin \mathbb{Z} \delta$, then there exists a Hall polynomial $\varphi_{Y / X, X}^{Y}=\varphi_{*, X}^{Y}$.

Proof. Since $Y$ is preprojective, so is $X$. Then by Lemma 5.25 the polynomial $\varphi_{*, X}^{Y}$ exists. By the lemma above we have $\varphi_{Y / X, X}^{Y}=\varphi_{*, X}^{Y}$.

Proposition 7.5. Let $X$ and $Y$ be indecomposable preprojective A-modules. Assume that $X$ is a GR-submodule of $Y$. If $\operatorname{rank} Y \geq 2$, then

(1) $\underline{\operatorname{dim}} Y / X \notin \mathbb{Z} \delta$; and

(2) There exists a Hall polynomial $\varphi_{Y / X, X}^{Y}$.

Proof. Assume that $\operatorname{rank} Y \geq 2$. Then $\Delta$ is not of type $A_{n}$.

(1) Assume that $\underline{\operatorname{dim}} Y / X \in \mathbb{Z} \delta$ and set $v:=\underline{\operatorname{dim}} X-\operatorname{dim} X(\infty) \delta$. Then $v_{\infty}=0$, $X=M(v+s \delta)$ and $Y=M(v+t \delta)$ for some $s<t$ in $\mathbb{N}$, and $\operatorname{rank} X=v_{1}=\operatorname{rank} Y$. For each $r \in \mathbb{N}$ we may assume that $M:=M(v+r \delta)$ has the following structure by [14, Theorems 2, 3]: $M\left(x_{i j}\right)=k^{v_{x_{i j}}+r}$ for all $x_{i j} \in Q_{0} ; M\left(\alpha_{1 j}\right)$ has the form $\left[\begin{array}{c}\mathbb{1}_{v_{x_{1, j+1}+r}} \\ 0\end{array}\right]$ for all $1 \leq j \leq p(1) ; M\left(\alpha_{2 j}\right)$ has the form $\left[\begin{array}{c}0 \\ \mathbb{1}_{v_{x_{2, j+1}}+r}\end{array}\right]$ for all $1 \leq j \leq p(2) ; M\left(\alpha_{32}\right)$ has the form $\left[\begin{array}{c}0 \\ \mathbb{1}_{r}\end{array}\right]$; and $M\left(\alpha_{31}\right)=-Z_{r}$, where $Z_{r}$ is the $r$-th enlargement (see [14, Section 2] for the definition) of a matrix $Z$ listed in [14, Theorem 3, Table 1] that is determined by $v$ not depending on $r$ (only here we use the assumption that char $k \neq 2$ ). For all $r<r^{\prime}$ in $\mathbb{N}$ we can define a monomorphism $f: M(v+r \delta) \rightarrow M\left(v+r^{\prime} \delta\right)$ by setting $f_{x}:=\left[\begin{array}{c}\mathbb{1}_{v_{x}+r} \\ 0\end{array}\right]$ for all $x \in Q_{0}$, which we can regard the inclusion $M(v+r \delta) \hookrightarrow$ $M\left(v+r^{\prime} \delta\right)$. Now if $t-s>1$, then we have strict inclusions of indecomposable modules $X=M(v+s \delta) \hookrightarrow M(v+(s+1) \delta) \hookrightarrow M(v+t \delta)=Y$, which contradicts the fact that the inclusion $X \hookrightarrow Y$ is mono-irreducible (Proposition 7.2). Hence we must have $t=s+1$. Then a direct calculation shows that Coker $f=Y / X$ has the following 
structure: $(Y / X)(x)=k$ for all $x \in Q_{0}$; and

$$
(Y / X)\left(\alpha_{i j}\right)= \begin{cases}0 & \text { if } i=1 \text { and } v_{1 j}=v_{1, j+1} \\ -\mathbb{1} & \text { if }(i, j)=(3,1) \\ \mathbb{1} & \text { otherwise }\end{cases}
$$

for all $\alpha_{i j} \in Q_{1}$. Since $v_{1}=\operatorname{rank} Y \geq 2$, we see $(Y / X)\left(\alpha_{1 j}\right)=0$ for at least two distinct values of $j$, which shows that $Y / X$ is decomposable, a contradiction to Proposition 7.2. Hence we must have $\underline{\operatorname{dim}} Y / X \notin \mathbb{Z} \delta$.

(2) This follows from (1) by Proposition 7.4.

7.b. Proof of Proposition 4.8. We will make full use of the following fundamental facts on simple Lie algebras below: Let $0 \neq x \in \mathfrak{g}(\Delta)_{\alpha}$ and $0 \neq y \in \mathfrak{g}(\Delta)_{\beta}$ for some $\operatorname{roots} \alpha, \beta$ of $\mathfrak{g}(\Delta)$, and assume that $\alpha+\beta$ is a root of $\mathfrak{g}(\Delta)$. Then $0 \neq[x, y] \in \mathfrak{g}(\Delta)_{\alpha+\beta}$.

First we show that Proposition 4.8 has a slightly stronger form for a positive root $v$ of $\chi_{A}$ with $M(v)$ a regular module.

Lemma 7.6. Let $M$ be a non-sincere indecomposable A-module. Then $\mathbf{u}_{[M]} \in L(A)_{1}$ and $0 \neq \phi^{-1}\left(\overline{\mathbf{u}}_{[M]}\right) \in \mathfrak{g}(\Delta)_{\operatorname{deg} M}$.

Proof. We already know that $\mathbf{u}_{[M]} \in L(A)_{1}$ by Proposition 3.14. By induction on $\operatorname{dim} M$ we show that $0 \neq \phi^{-1}\left(\overline{\mathbf{u}}_{[M]}\right) \in \mathfrak{g}(\Delta)_{\operatorname{deg} M}$. Assume first that $\operatorname{dim} M=1$. Then $\overline{\mathbf{u}}_{[M]}=\varepsilon_{x}$ for some $x \in Q_{0}$. If $x \neq \infty$ then $0 \neq \phi^{-1}\left(\overline{\mathbf{u}}_{[M]}\right)=E_{x} \in \mathfrak{g}(\Delta)_{e_{x}}=\mathfrak{g}(\Delta)_{\operatorname{deg} M}$ and the assertion holds. If $x=\infty$, then we know that $\varepsilon_{\infty}=\frac{1}{c}\left[\zeta_{x_{1}}, \ldots, \zeta_{x_{n}}\right]$ for some $c \in \mathbb{Z}^{\times}$and for some permutation $\left(x_{1}, \ldots, x_{n}\right)$ of $\Delta_{0}$ as in the proof of surjectivity of $\phi$. Hence

$$
\phi^{-1}\left(\overline{\mathbf{u}}_{[M]}\right)=\frac{1}{c}\left[F_{x_{1}}, \ldots, F_{x_{n}}\right] \in \mathfrak{g}(\Delta)_{e_{\infty}-\delta} \backslash\{0\}=\mathfrak{g}(\Delta)_{\operatorname{deg} M} \backslash\{0\},
$$

and the assertion holds in this case. Assume next that $\operatorname{dim} M>1$. Then as in the proof of Proposition 3.14 there is a non-sincere indecomposable $A$-module $N$ (with $\operatorname{dim} N=\operatorname{dim} M-1)$ and a simple $A$-module $S$ such that $\overline{\mathbf{u}}_{[M]}= \pm\left[\overline{\mathbf{u}}_{[S]}, \overline{\mathbf{u}}_{[N]}\right]$ in any case. Here $0 \neq \phi^{-1}\left(\overline{\mathbf{u}}_{[S]}\right) \in \mathfrak{g}(\Delta)_{\operatorname{deg} S}$, and by the induction hypothesis $0 \neq \phi^{-1}\left(\overline{\mathbf{u}}_{[N]}\right) \in$ $\mathfrak{g}(\Delta)_{\operatorname{deg} N}$. Since $\operatorname{deg} S+\operatorname{deg} N=\operatorname{deg} M$ is a root of $\mathfrak{g}(\Delta)$ by Lemma 4.6, we have $0 \neq \phi^{-1}\left(\overline{\mathbf{u}}_{[M]}\right)= \pm\left[\phi^{-1}\left(\overline{\mathbf{u}}_{[S]}\right), \phi^{-1}\left(\overline{\mathbf{u}}_{[N]}\right)\right] \in \mathfrak{g}(\Delta)_{\operatorname{deg} M}$.

Proposition 7.7. Let $v$ be a positive root of $\chi_{A}$. If $M(v)$ is regular, then

(1) $\mathbf{u}_{m(v)} \in L(A)_{1} \backslash I(A)$;

(2) $\phi^{-1}\left(\overline{\mathbf{u}}_{m(v)}\right) \in \mathfrak{g}(\Delta)_{\operatorname{deg} v}$; and

(3) $\overline{\mathbf{u}}_{m(v+\delta)}=\overline{\mathbf{u}}_{m(v)}$.

Proof. There exists a unique regular exceptional module $X$ such that $v^{\prime}:=\underline{\operatorname{dim}} X$ has the property that $v-v^{\prime} \in \mathbb{Z} \delta$. Then $\operatorname{deg} v=\operatorname{deg} v^{\prime}=\operatorname{deg}(v+\delta)$. By Proposition 5.18 both $\mathbf{u}_{m(v)}-\mathbf{u}_{m\left(v^{\prime}\right)}$ and $\mathbf{u}_{m(v+\delta)}-\mathbf{u}_{m\left(v^{\prime}\right)}$ are in $I(A)$. By Remark 5.13 we have $\mathbf{u}_{m\left(v^{\prime}\right)} \in L(A)_{1}$ because $M\left(v^{\prime}\right)$ is non-sincere. Hence $\mathbf{u}_{m(v)}, \mathbf{u}_{m\left(v^{\prime}\right)}, \mathbf{u}_{m(v+\delta)} \in L(A)_{1}$ and $\overline{\mathbf{u}}_{m(v+\delta)}=\overline{\mathbf{u}}_{m(v)}=\overline{\mathbf{u}}_{m\left(v^{\prime}\right)}$. By the lemma above we have $\mathbf{u}_{m(v)} \notin I(A)$ and $\phi^{-1}\left(\overline{\mathbf{u}}_{m(v)}\right)=$ $\phi^{-1}\left(\overline{\mathbf{u}}_{m\left(v^{\prime}\right)}\right) \in \mathfrak{g}(\Delta)_{\operatorname{deg} v^{\prime}}=\mathfrak{g}(\Delta)_{\operatorname{deg} v}$. 
Remark 7.8. The statements above clearly hold also for $v=e_{x}+t \delta$ for all $x \in Q_{0}$ and $t \in \mathbb{N}_{0}$.

Proof of Proposition 4.8 in general. Let $v$ be a positive root of $\chi_{A}$. We have to prove the following:

(1) $\overline{\mathbf{u}}_{m(v)} \in L(A)_{1}^{\mathbb{C}} / I(A)^{\mathbb{C}}$

(2) $0 \neq \phi^{-1}\left(\overline{\mathbf{u}}_{m(v)}\right) \in \mathfrak{g}(\Delta)_{\operatorname{deg} v}$; and

(3) $\mathbb{C} \overline{\mathbf{u}}_{m(v+\delta)}=\mathbb{C} \overline{\mathbf{u}}_{m(v)}$.

If both (1) and (2) are shown, then we have $\mathbb{C} \overline{\mathbf{u}}_{m(v)}=\phi\left(\mathfrak{g}(\Delta)_{\operatorname{deg} v}\right)$. Then the equality $\operatorname{deg}(v+\delta)=\operatorname{deg} v$ proves the statement (3). Hence it is enough to show the statements (1) and (2) by induction on $\operatorname{dim} M(v)$. Set $M:=M(v)$. If $\operatorname{dim} M=1$, then $M$ is non-sincere and both (1) and (2) hold by Lemma 7.6. Suppose next that $\operatorname{dim} M>1$. Assume that both (1) and (2) hold for all positive root $w$ of $\chi_{A}$ with $\operatorname{dim} M(w)<$ $\operatorname{dim} M$.

Case 1. $M$ is regular. In this case the assertion is already proved in the previous proposition.

Case 2. $M$ is preprojective. If $\operatorname{rank} M=1$, then by looking at the structure of $M$ described in 14 it is easy to see that there exists an indecomposable maximal submodule $X$ of $M$. Set $S:=M / X$. Then $X$ is also preprojective and $\operatorname{rank} X=1$. By setting $v^{\prime}:=\underline{\operatorname{dim}} X$ we may write $X=M\left(v^{\prime}\right)$. Since $\operatorname{rank} S=\operatorname{rank} M-\operatorname{rank} X=0, S$ is a regular simple $A$-module, and has the form $S=M\left(e_{x}\right)$ for some $x \in Q_{0} \backslash\{1, \infty\}$. A direct calculation shows that $\mathbf{u}_{[M]}=\left[\mathbf{u}_{[S]}, \mathbf{u}_{[X]}\right]$. Thus $\overline{\mathbf{u}}_{m(v)}=\left[\overline{\mathbf{u}}_{m\left(e_{x}\right)}, \overline{\mathbf{u}}_{m\left(v^{\prime}\right)}\right]$. By the induction hypothesis we have $\overline{\mathbf{u}}_{m\left(e_{x}\right)}, \overline{\mathbf{u}}_{m\left(v^{\prime}\right)} \in L(A)_{1}^{\mathbb{C}} / I(A)^{\mathbb{C}}$ and $0 \neq \phi^{-1}\left(\overline{\mathbf{u}}_{m\left(e_{x}\right)}\right) \in$ $\mathfrak{g}(\Delta)_{\operatorname{deg} e_{x}}, 0 \neq \phi^{-1}\left(\overline{\mathbf{u}}_{m\left(v^{\prime}\right)}\right) \in \mathfrak{g}(\Delta)_{\operatorname{deg} v^{\prime}}$. Hence $\overline{\mathbf{u}}_{m(v)} \in L(A)_{1}^{\mathbb{C}} / I(A)^{\mathbb{C}}$ and $\phi^{-1}\left(\overline{\mathbf{u}}_{m(v)}\right)=$ $\left[\phi^{-1}\left(\overline{\mathbf{u}}_{m\left(e_{x}\right)}\right), \phi^{-1}\left(\overline{\mathbf{u}}_{m\left(v^{\prime}\right)}\right)\right] \in \mathfrak{g}(\Delta)_{\operatorname{deg} v} \backslash\{0\}$ because $\operatorname{deg} e_{x}+\operatorname{deg} v^{\prime}=\operatorname{deg} v$ is a root of $\mathfrak{g}(\Delta)$ by Proposition 4.6. Hence both (1) and (2) hold. Therefore we may assume that rank $M \geq 2$.

Let $L$ be a GR-submodule of $M$ and $N:=M / L$. Then both $L$ and $N$ are indecomposable. Set $v^{\prime}:=\underline{\operatorname{dim}} L, v^{\prime \prime}:=\underline{\operatorname{dim}} N$. In this case $L$ is also preprojective and $L=M\left(v^{\prime}\right)$. By Proposition 7.5 we have $v^{\prime \prime} \notin \mathbb{Z} \delta$ and there exists a Hall polynomial $\varphi_{N L}^{M}$. Hence $a \mathbf{u}_{m(v)}=\left[\mathbf{u}_{m\left(v^{\prime \prime}\right)}, \mathbf{u}_{m\left(v^{\prime}\right)}\right]$ for some $a \in \mathbb{Z}$. Here both $v^{\prime}$ and $v^{\prime \prime}$ are positive roots of $\chi_{A}$ with $\operatorname{dim} M\left(v^{\prime}\right), \operatorname{dim} M\left(v^{\prime \prime}\right)<\operatorname{dim} M$. Therefore by the induction hypothesis, both (1) and (2) hold for $v^{\prime}, v^{\prime \prime}$. Then by the statement (1) for $v^{\prime}, v^{\prime \prime}$ we have $a \overline{\mathbf{u}}_{m(v)}=\left[\overline{\mathbf{u}}_{m\left(v^{\prime \prime}\right)}, \overline{\mathbf{u}}_{m\left(v^{\prime}\right)}\right] \in L(A)_{1}^{\mathbb{C}} / I(A)^{\mathbb{C}}$, and by (2) for $v^{\prime}, v^{\prime \prime}$ we obtain $a \phi^{-1}\left(\overline{\mathbf{u}}_{m(v)}\right)=\left[\phi^{-1}\left(\overline{\mathbf{u}}_{m\left(v^{\prime \prime}\right)}\right), \phi^{-1}\left(\overline{\mathbf{u}}_{m\left(v^{\prime}\right)}\right)\right] \in \mathfrak{g}(\Delta)_{\operatorname{deg} v} \backslash\{0\}$ because $\operatorname{deg} v^{\prime \prime}+\operatorname{deg} v^{\prime}=\operatorname{deg} v$ is a root of $\mathfrak{g}(\Delta)$ by Proposition 4.6. Thus $a \neq 0$ and we finally have both (1) and (2) for $v$.

Case 3. $M$ is preinjective. The dual argument works to show the assertion.

\section{Example}

8.a. Basis vectors. For $\Delta=D_{5}$ we exhibit basis vectors of positive and negative parts of $L(A)_{1}^{\mathbb{C}} / I(A)^{\mathbb{C}} \cong \mathfrak{g}(\Delta)$ in the Auslander-Reiten quiver of $A$. The positive part has 20 basis vectors: 15 vectors are in the preprojective component (Fig.8.1) and 5 vectors are in the non-homogeneous tubes (Figs.8.3 and 8.4). Similarly the negative 


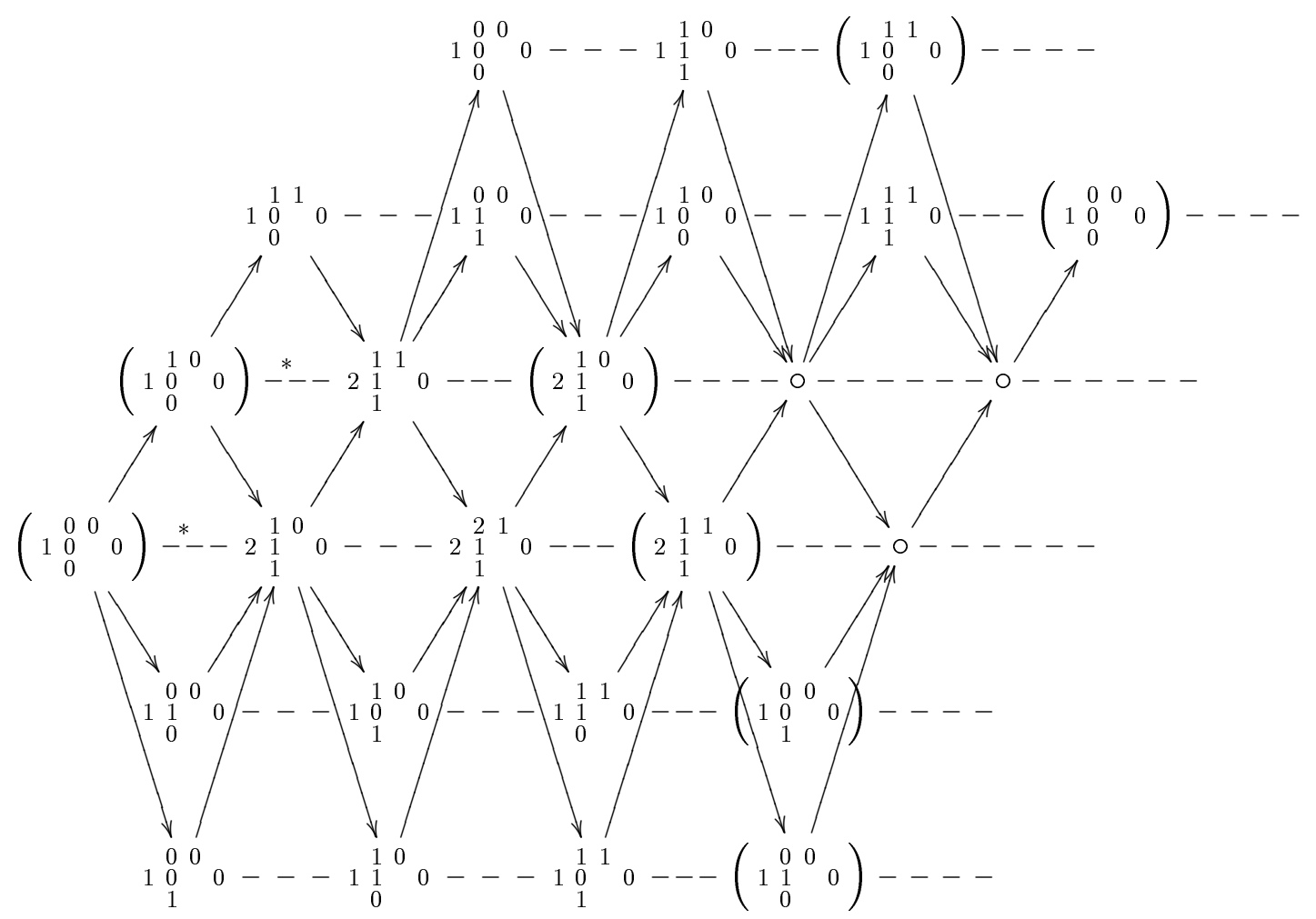

FiguRE 8.1. 15 basis vectors in the preprojective component

part has also 20 basis vectors: 15 vectors are in the preinjective component (Fig.8.2) and 5 vectors are in the non-homogeneous tubes. In Fig 8.1 vectors corresponding to indecomposable $A$-modules $M$ are given by their degrees $\underline{\operatorname{dim}} M-\operatorname{dim} M(\infty) \delta$ (see Definition 4.5), the broken lines stand for the Auslander-Reiten translation (from the right to the left as usual); those with $*$ indicate that the transformations from the left to the right are not given by the matrix $\Phi^{-1}$, whereas those without $*$ indicate that the same transformations are given by $\Phi^{-1}$. Vectors that are not chosen as representatives of basis elements are written in parentheses. The vectors in parentheses such that the same appear already on their left show us the action of $\Phi^{-1}$ on $\underline{\operatorname{dim}} \mathcal{P}_{r}$ (see 3.11). The dual remarks work for Fig.8.2. In Figures 8.3 and 8.4 the parallel arrows drawn by double lines should be identified to form tubes.

Remark 8.1. In general the preprojective (resp. preinjective) component over a domestic canonical algebra contains only basis vectors of the positive (resp. negative) part because the dimension vector of each preprojective (resp. preinjective) module takes the minimum (resp. maximum) value at the vertex $\infty$ (see Remark 4.7 for detail).

8.b. $E_{8}$ case. By Proposition 4.8 (3) we see that if $v$ is a positive root of $\chi_{A}$, then $\overline{\mathbf{u}}_{m(v+\delta)}=r_{v} \overline{\mathbf{u}}_{m(v)}$ for some $r_{v} \in \mathbb{C}^{\times}$. For a positive root $v$ of $\chi_{A}$ with $M(v)$ simple or regular we know that $r_{v}=1$. Here we exhibit an example for $\Delta=E_{8}$ showing that this is not always the case. 


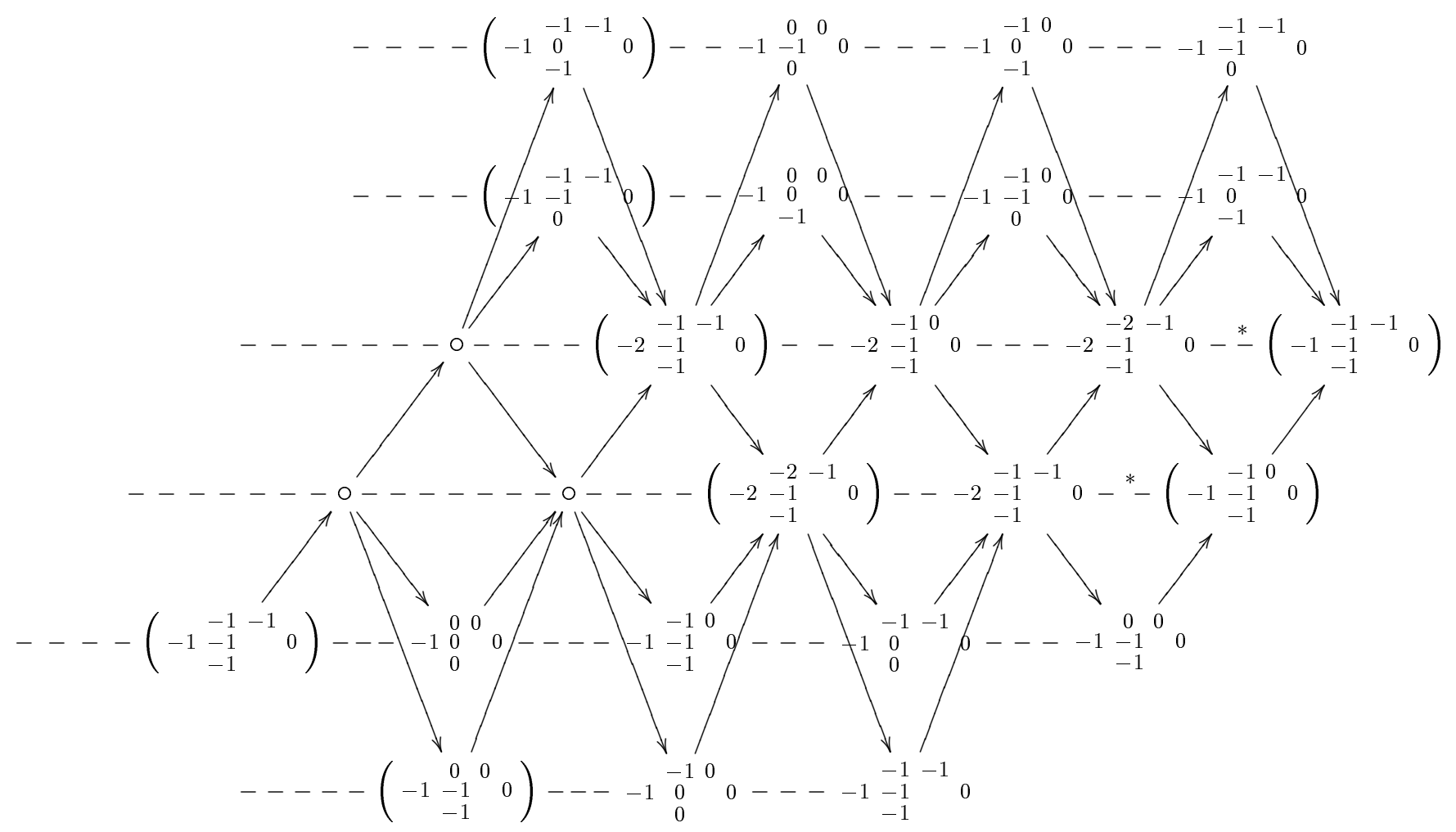

FIgURE 8.2. 15 basis vectors in the preinjective component

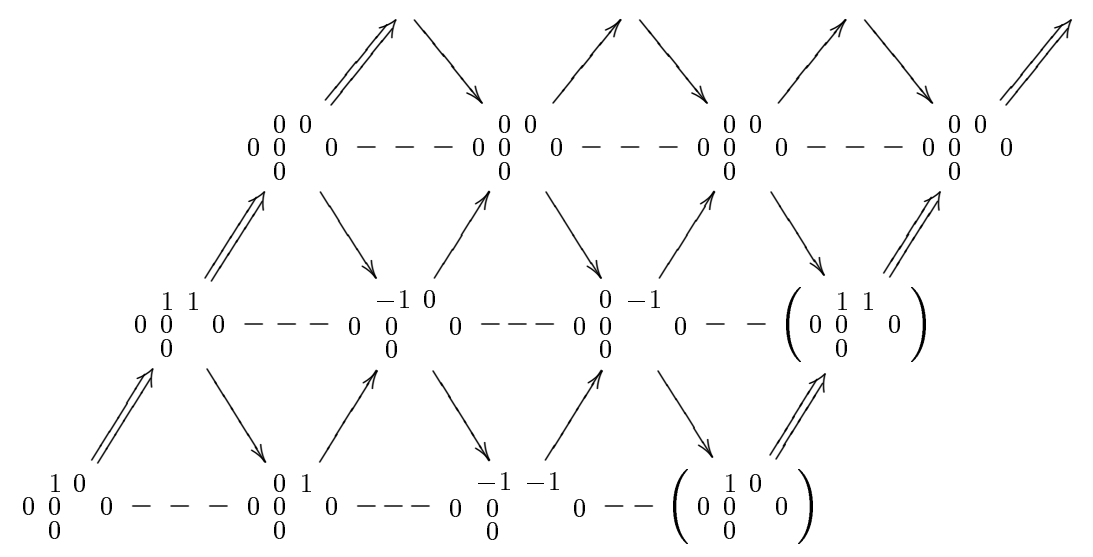

Figure 8.3. Tube of rank 3

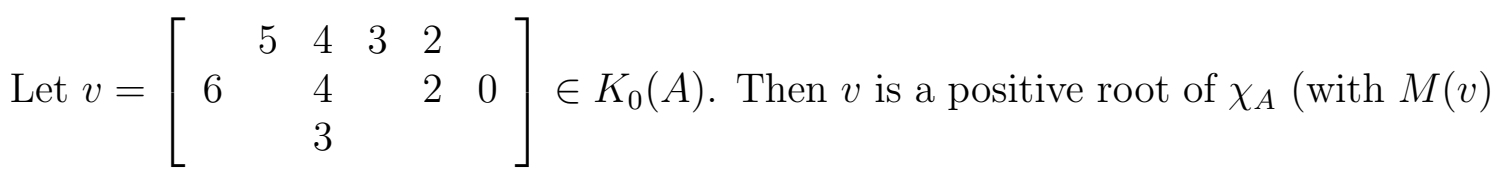
exceptional), and so are $v+e_{\infty}=\left[\begin{array}{rrrrrr} & 5 & 4 & 3 & 2 & \\ 6 & & 4 & & 2 & 1 \\ & & 3 & & & \end{array}\right]$ and $\operatorname{deg}\left(v+e_{\infty}\right)=v+e_{\infty}-\delta=$ 

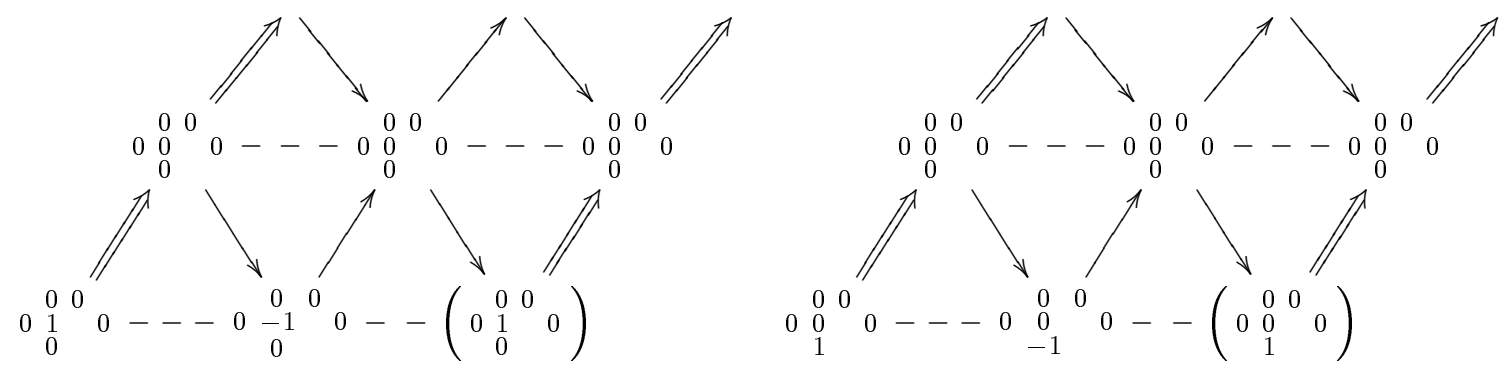

Figure 8.4. Tubes of rank 2

$\left[\begin{array}{rrrrr} & 4 & 3 & 2 & 1 \\ 5 & 3 & & 1 & 0 \\ & & 2 & & \end{array}\right]$. A direct calculation shows that $\left[\mathbf{u}_{m\left(e_{\infty}\right)}, \mathbf{u}_{m(v)}\right]=\mathbf{u}_{m\left(v+e_{\infty}\right)}$ but
$\left[\mathbf{u}_{m\left(e_{\infty}\right)}, \mathbf{u}_{m(v+\delta)}\right]=-\mathbf{u}_{m\left(v+e_{\infty}+\delta\right)}$. Hence we have $r_{v+e_{\infty}}=-r_{v}$, and at least one of these cannot be 1 .

In the first version of this paper we assumed that $I(A)$ contains the differences $\mathbf{u}_{m(v+t \delta)}-\mathbf{u}_{m(v)}$ (namely we assumed that $r_{v+(t-1) \delta}=1$ ) for all $v$ with $M(v)$ exceptional and $t \in \mathbb{N}$, and we found a serious error that $L(A)_{1}^{\mathbb{C}} / I(A)^{\mathbb{C}}=0$ in this case. The present version corrects this error.

\section{Acknowledgments}

The first version of this work was done while I was visiting the University of Bielefeld in 2003/2004. I would like to thank Professor Claus M. Ringel for suggesting me the usefulness of canonical algebras, and for answering my questions on their representations. I am grateful to Osaka City University for the support of the visit. I would also like to thank Bangming Deng, Christof Geiß, Andrew Hubery, and Dirk Kussin for helpful discussions to prove Theorem 6.1 in the first version although that is not used in this version. The first version was announced at ICRA XI held in Patzcuaro, Mexico in 2004. An outline of the present version was announced at "Conference on Representation Theory and related topics" held at ICTP in Trieste, Italy in 2006.

\section{REFERENCES}

[1] Asashiba, H.: Realization of general and special linear algebras via Hall algebras, preprint.

[2] Asashiba, H.: Realization of simple Lie algebras via Hall algebras of tame hereditary algebras, preprint.

[3] Auslander, M., Reiten, I. and Smalø, S.O.: Representation Theory of Artin Algebras, Cambridge studies in advanced mathematics 36, Cambridge University Press.

[4] Bongartz, K.: Critical simply connected algebras, Manuscripta Math. 46, no. 1-3, 117-136, 1984.

[5] Chen, Zh.: Canonical tubular algebras and affine Kac-Moody algebras, preprint.

[6] Gabriel, P.: Unzerlegbare Darstellungen I, Man. Math. 6, 71-103, 1972.

[7] Gabriel, P.: Auslander-Reiten sequences and representation-finite algebras. Representation theory, I (Proc. Workshop, Carleton Univ., Ottawa, Ont., 1979) Springer Lect. Notes Math. 831, $1-71,1980$.

[8] Grothendieck, A.: Groupes des classes des categories abeliennes et triangulees, Complexes parfait, In: SGA 5, Expose VIII, Springer Lect. Notes 589, 1977. 
[9] Happel, D.: Triangulated categories in the representation theory of finite dimensional algebras, London Math. Soc. Lect. Notes 119, Cambridge Univ. Press, 1988.

[10] Happel, D. and Ringel, C. M.: Tilted algebras, Trans. Amer. Math. Soc. 274, 399-443, 1982.

[11] Happel, D. and Vossieck, D.:Minimal algebras of infinite representation type with preprojective component, Manuscripta Math. 42, no. 2-3, 221-243, 1983.

[12] Iyama, O.: On Hall algebras of hereditary orders, Comm. Alg. 32, no. 4, 1571-1579, 2004.

[13] Kac, V.G.: Infinite dimensional Lie algebras, third edition, Cambridge Univ. Press, 1990.

[14] Kussin, D. and Meltzer, H.: Indecomposable modules for domestic canonical algebras, preprint.

[15] Ovsienko, S. A.: Integral weakly positive forms, In: Schur matrix problems and quadratic forms, Kiev, 3-17, 1978.

[16] Peng, L.: Some Hall polynomials for representation-finite trivial extension algebras, J. Alg. 197, 1-13, 1997.

[17] Peng, L. and Xiao, J.: Root categories and simple Lie algebras, J. Alg. 198, 19-56, 1997.

[18] Peng, L. and Xiao, J.: Triangulated categories and Kac-Moody algebras, Invent. Math. 140, 563-603, 2000.

[19] Rickard, J.: Morita theory for derived categories, J. London Math. Soc. (2) 39, 436-456, 1989.

[20] Riedtmann, Ch.: Lie algebras generated by indecomposables, J. Alg. 170, 526-546, 1994.

[21] Ringel, C. M.: Tame algebras and integral quadratic forms, Springer Lect. Notes in Math. 1099, 1984.

[22] Ringel, C. M.: Canonical algebras (with an appendix by W.W.Crawley-Boevey), in "Topics in Algebra," Banach Center Publ. 26(1), Warszawa, 407-432, 1990.

[23] Ringel, C. M.: Hall algebras, in "Topics in Algebra," Banach Center Publ. 26(1), Warszawa, 433-447, 1990.

[24] Ringel, C. M.: Hall polynomials for the representation-finite hereditary algebras, Adv. Math. 84 (2), 137-178, 1990.

[25] Ringel, C. M.: From representations of quivers via Hall and Loewy algebras to quantum groups, In: Proc. Novosibirsk Conf. Algebra 1989, Contemporary Math. 131, 381-401, 1992.

[26] Ringel, C. M.: Lie algebras arising in representation theory, London Math. Soc. Lec. Notes 168, 248-291, 1992.

[27] Ringel, C. M.: The braid group action on the set of exceptional sequences of a hereditary artin algebra, Contemporary Math. 171, 339-352, 1994.

[28] Ringel, C. M.: Foundation of the representation theory of artin algebras, using the Gabriel-Roiter measure, Proc. of the 11-th International Conference on Representations of Algebras, Queretaro 2004.

[29] Szántó, C.: The Hall product of a general regular and a preprojective Kronecker module, preprint.

Department of Mathematics,

Faculty of Science,

Shizuoka University,

836 Ohya, Suruga-ku,

Shizuoka, 422-8529, Japan

E-mail address: shasash@ipc.shizuoka.ac.jp 